University of Tennessee Health Science Center

UTHSC Digital Commons

\title{
Molecular Response of Retinal Pigment Epithelial Cells to Oxidized Lipoproteins: Global and Targeted Studies
}

\author{
Diwa Koirala \\ University of Tennessee Health Science Center
}

Follow this and additional works at: https://dc.uthsc.edu/dissertations

Part of the Medicine and Health Sciences Commons

\footnotetext{
Recommended Citation

Koirala, Diwa (http://orcid.org/https://orcid.org/0000-0001-7950-0259), "Molecular Response of Retinal Pigment Epithelial Cells to Oxidized Lipoproteins: Global and Targeted Studies" (2018). Theses and Dissertations (ETD). Paper 473. http://dx.doi.org/10.21007/etd.cghs.2018.0466.
}

This Dissertation is brought to you for free and open access by the College of Graduate Health Sciences at UTHSC Digital Commons. It has been accepted for inclusion in Theses and Dissertations (ETD) by an authorized administrator of UTHSC Digital Commons. For more information, please contact jwelch30@uthsc.edu. 


\title{
Molecular Response of Retinal Pigment Epithelial Cells to Oxidized Lipoproteins: Global and Targeted Studies
}

\begin{abstract}
Global-scale examinations of biological systems at the molecular level complement targeted approaches to scientific inquiry that focus on specific subsets of biomolecules, or on a single molecule of interest. In this dissertation, we utilized both the discovery-based approach to evaluate the proteomics workflows centered around mass spectrometry as the key technology, and the targeted approach to examine the molecular response of RPE due to oxidized lipoproteins (oxLDL) treatments. A crucial aspect in proteomics studies is the design of bioanalytical strategies that maximize coverage of the complex repertoire of a proteome. A comprehensive, unbiased examination of the proteome represents a powerful approach toward system-level insights into disease mechanisms. We evaluated the performance of bioanalytical platforms for profiling of the proteome in a biological system. We applied a discovery-based approach to evaluate the global transcriptome and proteome changes due to oxLDL treatment in ARPE-19 cells.
\end{abstract}

We studied the role of scavenger receptors CD36 and CD5L/AIM in ARPE-19 cells when induced with oxLDL. We compared three different multidimensional proteome fractionation platforms: polymeric reversed-phase liquid chromatography at high pH (PLRP), sodium dodecyl sulfate-polyacrylamide gel electrophoresis (SDS-PAGE), and isoelectric focusing (IEF) separations. We applied a liquid chromatography-mass spectrometry/mass spectrometry (LC-MS/MS) in a data-dependent setting and used bioinformatics for protein identification. The three platforms identified a total of 1043 proteins altogether. Among the three bioanalytical strategies, SDS-PAGE followed by LC-MS/MS provided the best coverage. We also evaluated another bioanalytical platform which consists of a highresolution mass spectrometer combined with nano-UPLC in a data-independent setting without pre-fractionation for oxLDL mediated proteome alteration in ARPE-19 cells.

This platform outperformed the SDS-PAGE based analytical platform in terms of proteome coverage as it identified around 2500 proteins, ca. 3-fold more proteins than the latter. Most importantly, this platform was able to perform label free quantification of differentially expressed proteome alteration. The platforms identified proteins with diverse physicochemical characteristics involved in various functional roles within the biological system.

Furthermore, we carried out the first comparative transcriptomic and proteomic study for the evaluation of oxLDL effects on ARPE-19 cells after a $4 \mathrm{~h}$ exposure. The treatment with oxLDL affected the regulation of more than 700 genes that were involved in regulation of cell cycle, oxidative stress, cholesterol efflux, circadian rhythm, NRF-2 pathways. However, LDL treatment alone did not induce the regulation of these pathways. The differential proteomic analysis found 41 proteins affected due to the oxLDL treatment. This study provided a foundation for a bioanalytical platform for identification and label-free quantification in the human retinal pigment epithelial cells (ARPE-19) proteome. The list of differentially expressed proteins due to oxLDL treatment identified in this study gives insights to the change in proteins that might be interrogated for their roles in pathogenesis of macular degeneration. These findings could give us targets to intervene in the pathogenesis of AMD progression in human for the development of better treatment and prevention against this degenerative disease.

Lastly, we studied the mechanistic role of scavenger receptors CD36 and CD5L/AIM in oxLDL uptake by ARPE-19 cells. We, for the first time, demonstrated the presence of scavenger receptor CD5L in ARPE-19 cell. The oxLDL uptake was primarily dependent on CD36, and both the CD5L/AIM and CD36 were seen to co-localize in the presence of oxLDL. Our results suggest a new dynamics on CD5L/AIM on the oxLDL uptake that was not seen in macrophages. The reduction in intracellular accumulation of oxLDL in the 
presence of extracellular recombinant CD5L/AIM is an interesting phenomenon as it has been recently shown the involvement of CD5L/AIM in autophagy.

\section{Document Type}

Dissertation

Degree Name

Doctor of Philosophy (PhD)

\section{Program}

Pharmaceutical Sciences

\section{Research Advisor}

Sarka Beranova-Giorgianni, Ph.D.

\section{Keywords}

Age Related Macular Degeneration, Bioanalytics, Mass Spectrometry, Proteomics, Retinal Pigment Epithelium

\section{Subject Categories}

Medicine and Health Sciences 
Molecular Response of Retinal Pigment Epithelial Cells to Oxidized Lipoproteins: Global and Targeted Studies

\author{
A Dissertation \\ Presented for \\ The Graduate Studies Council \\ The University of Tennessee \\ Health Science Center \\ In Partial Fulfillment \\ Of the Requirements for the Degree \\ Doctor of Philosophy \\ From The University of Tennessee
}

By

Diwa Koirala

December 2018 
Copyright (C) 2018 by Diwa Koirala. All rights reserved. 


\section{DEDICATION}

To my parents Subana Koirala and Dr. Kumar Prasad Koirala

for their love and faith in me.

To my husband Dr. Nobal Bikram Niraula for his constant support and dedication.

To my children Nishan and Nimisha, for being the sweetest child and putting a smile on my face with their presence. 


\section{ACKNOWLEDGEMENTS}

I would like to express my sincere gratitude to all the people who helped me realize my dream. My journey towards achievement of my doctorate degree would not have been possible without tremendous support and love that I received from everyone that I met within last six years.

First and foremost, I would like to thank my advisor Dr. Sarka BeranovaGiorgianni with deep gratitude for providing me an opportunity to pursue my $\mathrm{Ph}$.D under her supervision. I will be forever grateful for her continuous support, guidance and encouragement. I immensely appreciate her for not giving up on me and being the driving force in my academic life. I would also like to thank my co-advisor Dr. Francesco Giorgianni for his guidance and support during these six years. I appreciate the countless number of hours he spent teaching me research and guiding me through various projects. He was always available to discuss my research plans, results and manuscript preparation. No words would ever explain how grateful I am to my advisor and coadvisor for the support they provided me not only on my academics but also my personal life.

I would like to thank my thesis committee members, Dr. Tao Lowe, Dr. Ivan Gerling and Dr. Marko Radic for the time, and support they provide me taking time form their busy schedule. Furthermore, I want to thank Dr. Alessandro Iannaccone from Duke University. Their useful suggestions on my research problems help me shape my dissertation.

I would like to thank all the faculty at UTHSC, college of pharmacy. I would like to thank Dr. Kumar and Dr. Park for letting me use their lab facilities, the qPCR machine and fluorescence microscope. I would like to thank Dr. Lowe for allowing me to use centrifuge and microscope in our shared cell culture room. I would like to thank Dr. Yates for letting me use Licor to take my western blot images. I would like to thank Dr. Meibohm for letting me use thermocycler every time I needed it. I would like to thank Dr. Chauhan and Dr. Peters for letting me use their flow cytometer. I would like to thank all the faculty of College of Pharmacy at UTHSC for their support. I would like to acknowledge all the staff at College of Pharmacy for their support. Brenda Thornton, Corliss Finlay, Cynthia Crowe, Mary Newell, Sherri Jackson, Benita Williams, Connie Childs, Shelley Cannioto for their administrative help. I would also like to thank the Department of Pharmaceutical Sciences for providing me the opportunity and funding for my $\mathrm{PhD}$ program.

I would take an opportunity to thank my friends at UTHSC who were one of the most integral part of my PhD life. I would like to thank Dr. Ashit Trivedi for showing me the importance of balancing my work life and my social life. Thank you for showing me the fun side of life during my PhD. I thank Dr. Shajila Siricilla for being my pillar of strength at UTHSC, for listening and understanding me and lending me her ears during my stressful times. I would like to thank Dr. Dileep Janagham for being my go-to person 
for my problems. I would like to thank Sabina Ranjit for helping me with flow cytometry and qPCR and lending me support whenever I needed. I would like to thank Dr. Nivesh Mittal for his support on my initial days at UTHSC. I would like to thank all my friends Dr. Chetan Rathi, Dr. Jordan Toutounchian, Dr. Josiah Ryman, Dr. Hemlata Patil, Dr. Pradeep Lukka, Sri Chandana Damera, Santosh Wagh, Arif Rahaman, Pallabita Chaudhary for their wonderful company. A sincere thanks goes to my friend Uttam Satyal for reading my thesis and giving me feedback. A special thanks to Saurab Dulal for his help.

A special feeling of appreciations and gratitude goes to my husband Dr. Nobal Niraula without whom this $\mathrm{PhD}$ would not have been possible. I am in debt for his constant support, motivations and keeping my spirits always high during my lowest moments. I would like to thank my little brother for motivating me to move a little ahead whenever I felt I could not do anymore. I would like to thank my in-laws Gobardhan Niraula and Sarada devi Niraula for their encouragement and positive attitudes towards my PhD. I would like to express my deep gratitude for my parents Dr. Kumar Prasad Koirala and Subana Koirala, for their endless love, support and encouragement to do better in life. A special thanks goes to the love of my life, my son Nishan, for brightening my life with his presence and my sweetheart Nimisha, who would sleep during night letting her mommy finish this dissertation. 


\begin{abstract}
Global-scale examinations of biological systems at the molecular level complement targeted approaches to scientific inquiry that focus on specific subsets of biomolecules, or on a single molecule of interest. In this dissertation, we utilized both the discovery-based approach to evaluate the proteomics workflows centered around mass spectrometry as the key technology, and the targeted approach to examine the molecular response of RPE due to oxidized lipoproteins (oxLDL) treatments. A crucial aspect in proteomics studies is the design of bioanalytical strategies that maximize coverage of the complex repertoire of a proteome. A comprehensive, unbiased examination of the proteome represents a powerful approach toward system-level insights into disease mechanisms. We evaluated the performance of bioanalytical platforms for profiling of the proteome in a biological system. We applied a discovery-based approach to evaluate the global transcriptome and proteome changes due to oxLDL treatment in ARPE-19 cells. We studied the role of scavenger receptors CD36 and CD5L/AIM in ARPE-19 cells when induced with oxLDL.
\end{abstract}

We compared three different multidimensional proteome fractionation platforms: polymeric reversed-phase liquid chromatography at high $\mathrm{pH}$ (PLRP), sodium dodecyl sulfate-polyacrylamide gel electrophoresis (SDS-PAGE), and isoelectric focusing (IEF) separations. We applied a liquid chromatography-mass spectrometry/mass spectrometry (LC-MS/MS) in a data-dependent setting and used bioinformatics for protein identification. The three platforms identified a total of 1043 proteins altogether. Among the three bioanalytical strategies, SDS-PAGE followed by LC-MS/MS provided the best coverage. We also evaluated another bioanalytical platform which consists of a highresolution mass spectrometer combined with nano-UPLC in a data-independent setting without pre-fractionation for oxLDL mediated proteome alteration in ARPE-19 cells. This platform outperformed the SDS-PAGE based analytical platform in terms of proteome coverage as it identified around 2500 proteins, $c a$. 3-fold more proteins than the latter. Most importantly, this platform was able to perform label free quantification of differentially expressed proteome alteration. The platforms identified proteins with diverse physicochemical characteristics involved in various functional roles within the biological system.

Furthermore, we carried out the first comparative transcriptomic and proteomic study for the evaluation of oxLDL effects on ARPE-19 cells after a $4 \mathrm{~h}$ exposure. The treatment with oxLDL affected the regulation of more than 700 genes that were involved in regulation of cell cycle, oxidative stress, cholesterol efflux, circadian rhythm, NRF-2 pathways. However, LDL treatment alone did not induce the regulation of these pathways. The differential proteomic analysis found 41 proteins affected due to the oxLDL treatment. This study provided a foundation for a bioanalytical platform for identification and label-free quantification in the human retinal pigment epithelial cells (ARPE-19) proteome. The list of differentially expressed proteins due to oxLDL treatment identified in this study gives insights to the change in proteins that might be interrogated for their roles in pathogenesis of macular degeneration. These findings could 
give us targets to intervene in the pathogenesis of AMD progression in human for the development of better treatment and prevention against this degenerative disease.

Lastly, we studied the mechanistic role of scavenger receptors CD36 and CD5L/AIM in oxLDL uptake by ARPE-19 cells. We, for the first time, demonstrated the presence of scavenger receptor CD5L in ARPE-19 cell. The oxLDL uptake was primarily dependent on CD36, and both the CD5L/AIM and CD36 were seen to co-localize in the presence of oxLDL. Our results suggest a new dynamics on CD5L/AIM on the oxLDL uptake that was not seen in macrophages. The reduction in intracellular accumulation of oxLDL in the presence of extracellular recombinant CD5L/AIM is an interesting phenomenon as it has been recently shown the involvement of CD5L/AIM in autophagy. 


\section{TABLE OF CONTENTS}

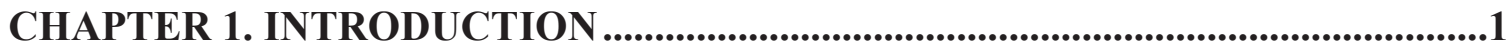

Dissertation Hypothesis and Specific Aims................................................................6

Aim 1. To Evaluate the Capabilities of Bioanalytical Platforms in Terms of

Extent and Depth of Proteome Coverage................................................................6

Aim 2. To Determine the Roles of Scavenger Receptors CD36 and CD5L/AIM in the Clearance of OxLDL by the Human Retinal Pigment Epithelial Cells ..............7

Aim 3. To Characterize OxLDL-induced Molecular Response in the RPE Cells.......7

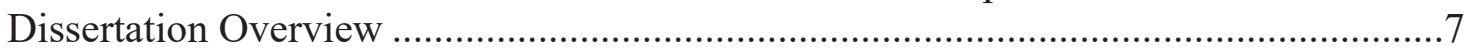

\section{CHAPTER 2. PROTEOMICS IN BIOLOGICAL SYSTEM: STUDY OF} BIOANALYTICAL PLATFORMS FOR BETTER PROTEOME COVERAGE ......8

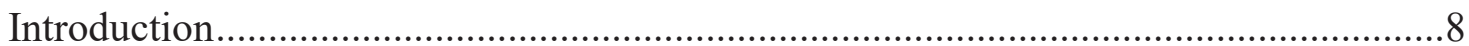

Mass Spectrometry Based Proteomics................................................................

Components of Mass Spectrometry Based Bioanalytical Proteomic Workflow .........9

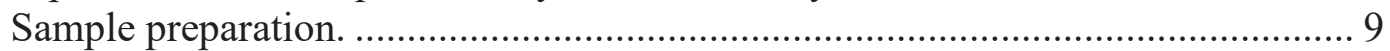

Protein/peptide separation................................................................................... 11

Liquid chromatography and mass spectrometry ………...................................... 13

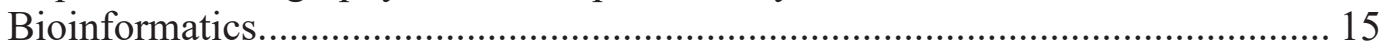

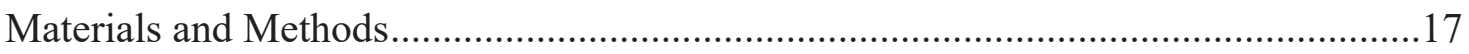

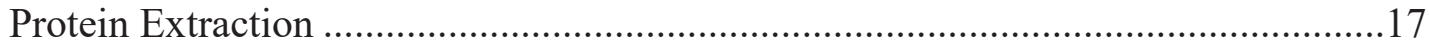

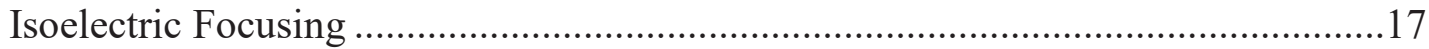

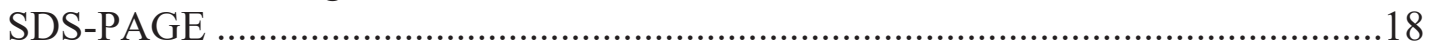

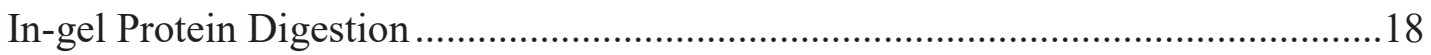

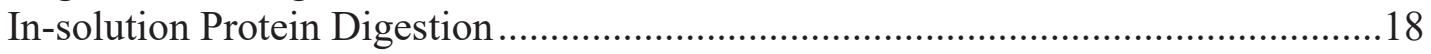

High-pH Reversed-Phase Chromatography (PLRP)..............................................19

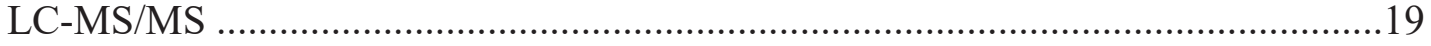

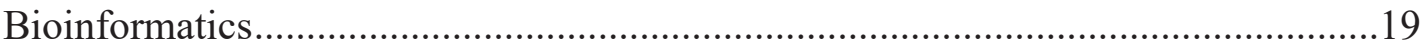

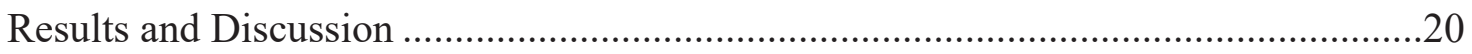

Summary of Protein Identification Results .............................................................2

Intrinsic Chemical Properties of Identified Proteins..................................................23

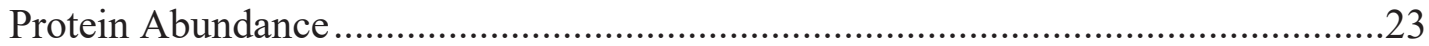

Categorization of the Identified Proteins .................................................................23

Selected Functional Characteristic of Mitochondrial Proteome Panel ......................28

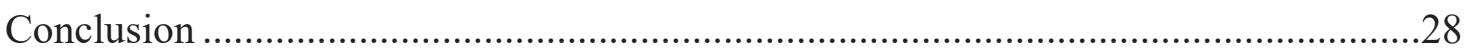

\section{CHAPTER 3. SCAVENGER RECEPTOR-MEDIATED UPTAKE AND} EFFECTS OF OXLDL IN RETINAL PIGMENT EPITHELIAL CELLS................36

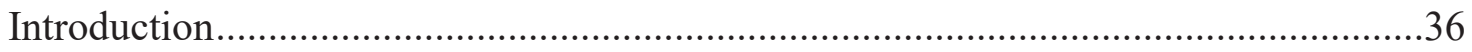

Pathophysiology of AMD: Genetic and Environmental Factors ................................38

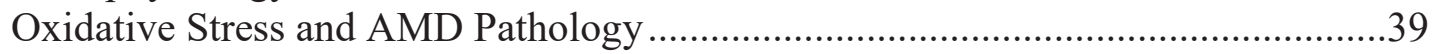

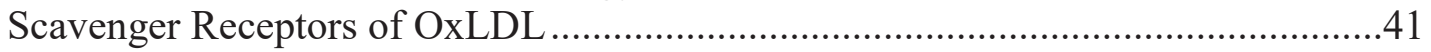

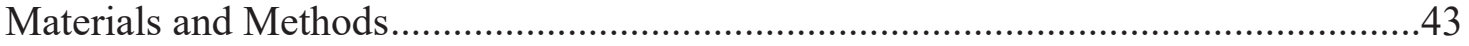

OxLDL Uptake by ARPE-19 Cells........................................................................4 
Western Blot Analysis .........................................................................................4

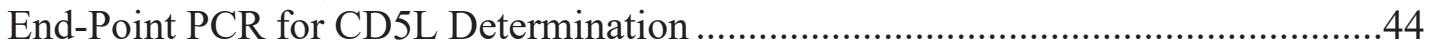

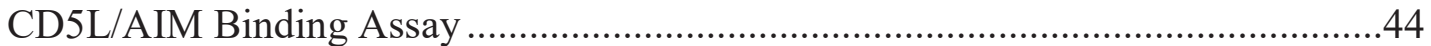

Cellular Uptake Assays of OxLDL by Confocal Microscopy and Flow

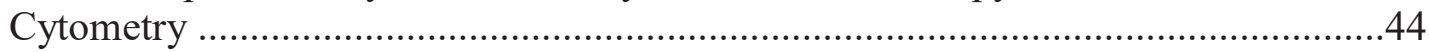

Blocking of CD36 Receptor for OxLDL Uptake Studies ..........................................4

qPCR Analysis for OxLDL Mediated Gene Expression ………………………........45

Induction of CD5L/AIM with LXR-Agonist...........................................................45

Cellular Uptake Assay of OxLDL by Flow Cytometry Using rhCD5L/AIM ............45

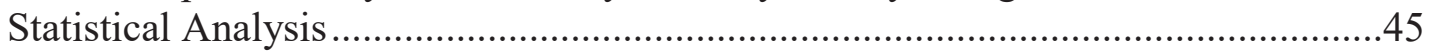

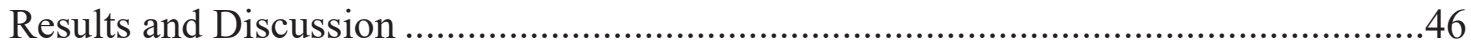

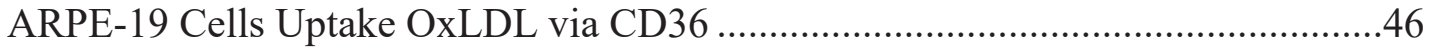

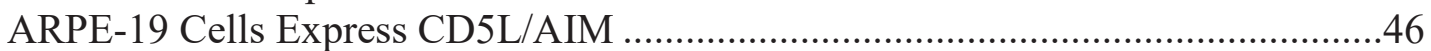

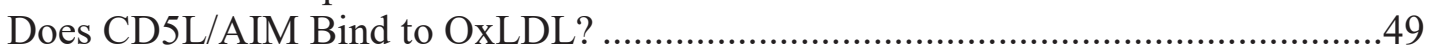

CD5L/AIM and CD36 Co-localize in the Presence of OxLDL .................................49

Effects of Anti-CD5L/AIM Antibody on OxLDL Uptake ........................................49

CD5L/AIM, CD36 Expression Changes Due to OxLDL Treatment..........................49

Effect of LXR Agonist on Expression of CD5L/AIM ...............................................54

Recombinant CD5L/AIM (rh-CD5L/AIM) Effects the OxLDL Uptake by

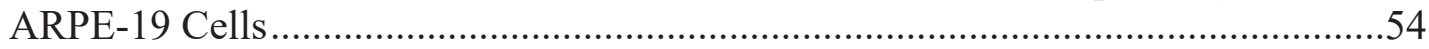

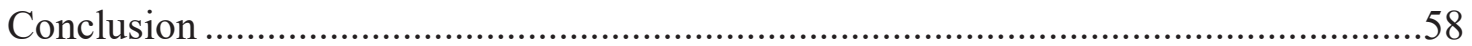

\section{CHAPTER 4. EARLY TRANSCRIPTOME ALTERATIONS INDUCED BY} OXLDL IN RETINAL PIGMENT EPITHELIUM CELLS .......................................59

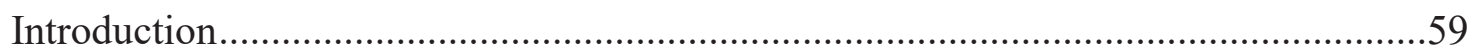

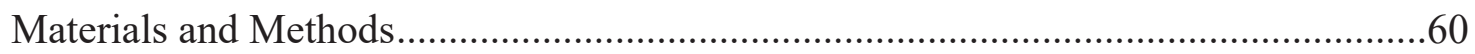

Cell Culture and RNA Preparation ..................................................................6

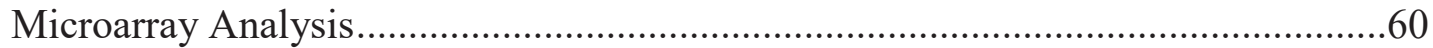

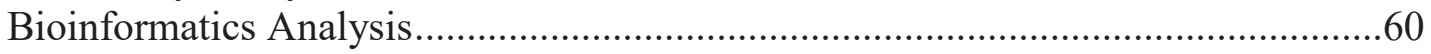

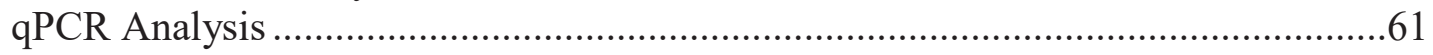

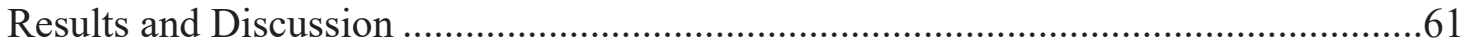

OxLDL and Anti-Oxidative Stress and Inflammatory Response ..............................70

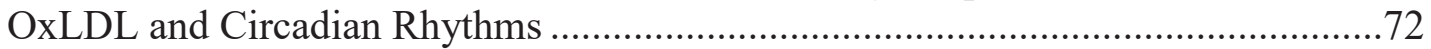

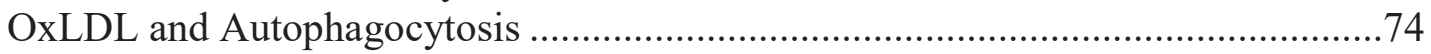

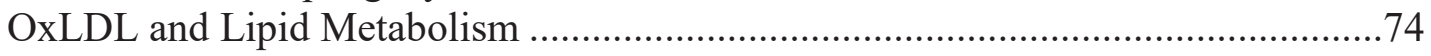

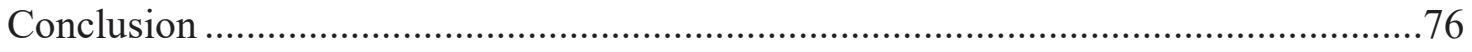

\section{CHAPTER 5. PROTEOMIC ANALYSIS OF OXLDL TREATED ARPE-19 CELLS: A STUDY OF LC-MS/MS BASED BIOANALYTICAL PLATFORMS ...78}

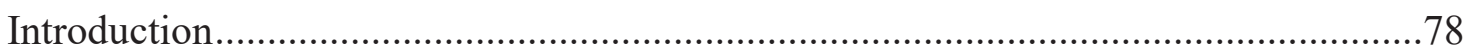

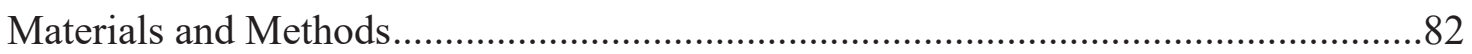

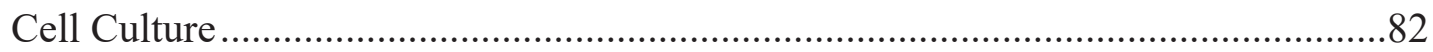

OxLDL Treatment and Sample Collection .............................................................8

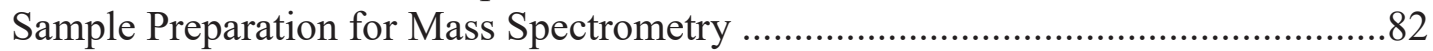

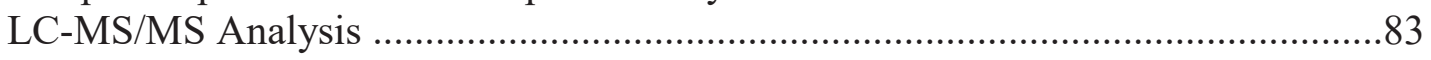

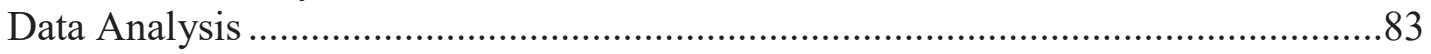




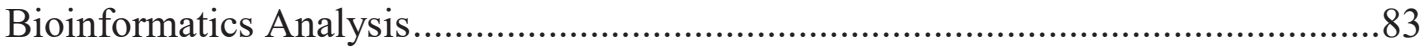

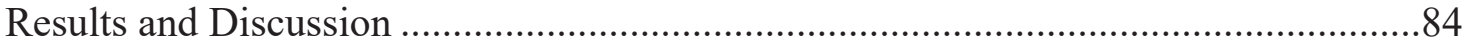

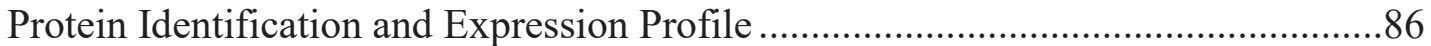

Differential Proteomic Profiling Due to OxLDL Treatment ....................................8

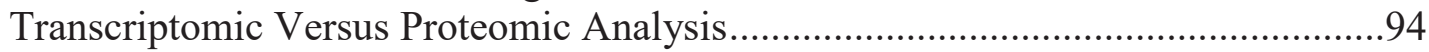

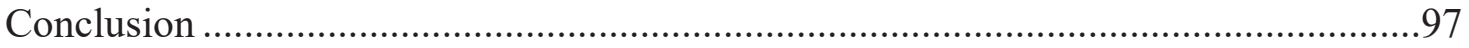

CHAPTER 6. CONCLUSION AND FUTURE DIRECTIONS ..................................98

LIST OF REFERENCES ....................................................................................................100

VITA 


\section{LIST OF TABLES}

Table 1-1. List of FDA approved/investigational drugs for dry and wet AMD..............3

Table 2-1. Total proteins identified in fractionated vs. unfractionated samples...........22

Table 2-2. Experimental design: numbers of samples for each platform.....................22

Table 2-3. Summary of the performances of three gel-based and gel-free bioanalytical platforms for mitochondrial proteome mapping....................24

Table 2-4. Representative proteins from selected functional classes..........................31

Table 3-1. Genes identified to be associated with AMD. .............................................40

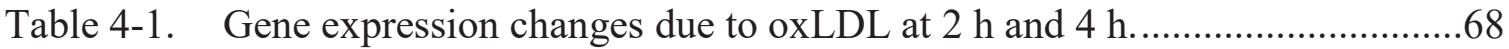

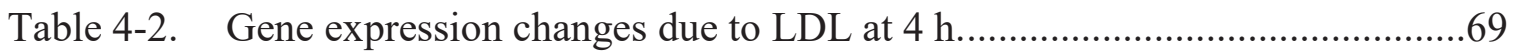

Table 4-3. Validation of gene expression changes by qPCR ....................................71

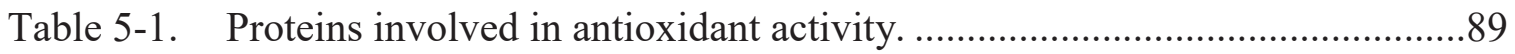

Table 5-2. Differentially expressed proteins due to oxLDL treatment in ARPE-19

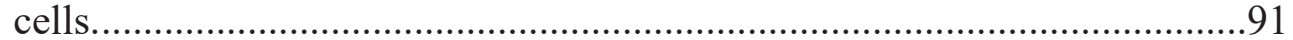

Table 5-3. List of common proteins/genes probed in microarray and proteomic studies. 


\section{LIST OF FIGURES}

Figure 1-1. RPE structure and location....................................................................

Figure 2-1. General scheme for proteomic analysis. ............................................. 10

Figure 2-2. An example of tandem MS/MS in a data dependent acquisition setting. .....16

Figure 2-3. Experimental scheme for the comparative evaluation of three bioanalytical platforms for mitochondrial proteome profiling...................21

Figure 2-4. Venn diagrams showing the distribution of unique proteins identified by each platform.

Figure 2-5. Distribution of intrinsic properties of the mitochondrial proteins identified by each platform.

Figure 2-6. Relative abundance of mitochondrial proteins sampled with each platform.

Figure 2-7. Gene ontology categorization of the identified proteins according to the biological process.

Figure 2-8. Distribution of proteins according to the presence of N-terminal mitochondrial targeting sequence (MTS).

Figure 3-1. Stages of AMD.

Figure 3-2. oxLDL uptake by ARPE-19 cells.

Figure 3-3. Expression of CD5L/AIM/AIM in the retinal pigment epithelium. . .48

Figure 3-4. CD5L/AIM-OxLDL binding assay. .50

Figure 3-5. Co-localization of CD36 and CD5L/AIM. 51

Figure 3-6. Effects of anti-CD5L/AIM antibody on the OxLDL uptake. 52

Figure 3-7. Effects of OxLDL on scavenger receptors.

Figure 3-8. Effect of T090 on ARPE-19 cells. 55

Figure 3-9. Effect of rh-CD5L/AIM on OxLDL uptake. 56

Figure 4-1. Cytoxicity of OxLDL in ARPE-19 cells

Figure 4-2. OxLDL for 2 and $4 \mathrm{~h}$ triggered a reproducible, robust gene expression response. 
Figure 4-3. Gene expression changes caused by oxLDL and LDL treatment.

Figure 4-4. Volcano plot and heat map for mRNA expression....................................66

Figure 4-5. Molecular networks and pathways...................................................67

Figure 4-6. OxLDL induced molecular change in regulation of circadian rhythm........75

Figure 5-1. Schematic representation of SYNAPT G2-Si mass spectrometry system....81

Figure 5-2. Schematic representation of bioanalytical workflow for proteomic analysis of oxLDL/LDL treated ARPE-19 cells.

Figure 5-3. Gene ontology categorization of the identified proteins according to (A) biological process, (B). molecular functions and (C). cellular components.

Figure 5-4. Venn diagram of proteins identified in ARPE-19 cells with control, LDL and oxLDL treatments. 


\section{LIST OF ABBREVIATIONS}

\begin{tabular}{|c|c|}
\hline$\mu \mathrm{g}$ & Microgram \\
\hline AMD & Age-Related Macular Degeneration \\
\hline AREDS & Age-Related Eye Disease Study \\
\hline ATCC & American Type Culture Collection \\
\hline apoB & Apolipoprotein B \\
\hline ARPE-19 & Adult Retinal Pigmented Epithelium Cells 19 \\
\hline $\mathrm{BRB}$ & Blood-Retinal Barrier \\
\hline BrM & Bruch’s Membrane \\
\hline CID & Collision Induced Dissociation \\
\hline CHAPS & Dimethyl[3-( propyl]. Azaniumyl\} propane-1-Sulfonate \\
\hline DAPI & 4',6-DiAmidino-2-PhenylIndole \\
\hline DHA & Docosahexaenoic Acid \\
\hline DMSO & Di Methyl Sulfoxide \\
\hline DTT & Dithiothreitol \\
\hline 2D-LC & Two-Dimensional Liquid Chromatography \\
\hline EDTA & Ethylene Diamine Tetra Acetic Acid \\
\hline DMEM & Dulbecco's Modified Eagle Medium \\
\hline ESI & Electrospray Ionization \\
\hline ETS & Electron Transport System \\
\hline FBS & Fetal Bovine Serum \\
\hline FDA & Food and Drug Administration \\
\hline FDR & False Discovery Rate \\
\hline FITC & Fluorescein Iso Thio Cyanate \\
\hline GlcNAc & N- Acetyl Glucosamine \\
\hline IEF & Isoelectric Focusing \\
\hline IPA & Ingenuity Pathway Analysis \\
\hline IPG & Immobilized pH Gradient \\
\hline LDL & Low Density Lipoprotein \\
\hline LC-MS & Liquid Chromatography - Mass Spectrometry \\
\hline $\mathrm{MS} / \mathrm{MS}$ & Tandem Mass Spectrometry \\
\hline MudPIT & Multidimensional Protein Identification Technology \\
\hline NEI & National Eye Institute \\
\hline OxLDL & Oxidized Low Density Lipoprotein \\
\hline OSEs & Oxidation-Specific Epitopes \\
\hline PLRP & Polymeric Reversed-Phase \\
\hline PCR & Polymerase Chain Reaction \\
\hline PBS & Phosphate Buffered Saline \\
\hline $\mathrm{pI}$ & Isoelectric Points \\
\hline PRRs & Pattern Recognition Receptors \\
\hline RF & Radio Frequency \\
\hline RPE & Retinal Pigment Epithelium \\
\hline RPC & Reversed Phase Chromatography \\
\hline RP-RP & Reversed Phase- Reversed Phase \\
\hline SCX & Strong Cation Exchange \\
\hline
\end{tabular}


SPE

SDS

SDS-PAGE

SSO

TFA

UPLC

$\mathrm{ZO}-1$
Solid Phase Extraction

Sodium Dodecyl Sulfate

Sodium Dodecyl Sulfate-Polyacrylamide Gel Electrophoresis

Sulfo-N-Succinimidyl Oleate

Trifluoroacetic Acid

Ultra Performance Liquid Chromatography

Zonula Occludens-1 


\section{CHAPTER 1. INTRODUCTION}

Global-scale examinations of biological systems at the molecular level complement targeted approaches to scientific inquiry that focus on specific subsets of biomolecules, for example components of a molecular pathways, or on a single molecule of interest such as a specific protein. The long-term research focus of our laboratory has been centered on proteomics. Research activities encompass development and optimization of bioanalytical workflows for proteomics as well as application of proteomics in various contexts including investigation of disease mechanisms and to discovery of new biomarkers. Proteomics is the large-scale study of structure and function of all the proteome, where proteome is defined as a set of proteins present in any biological system under a given condition. It provides a global insight into the abundance of protein expression, its localization, and interaction. Recent development of advanced proteomics tools has allowed scientists to perform large-scale, high throughput screening, detection, identification and analysis of proteins in a complex biological system.

Moreover, advances in mass spectrometric based proteomic techniques, along with protein fractionation and labeling techniques have allowed identification of less abundant proteins and post translational modification in the proteins possible. These developments and improvements in proteomic techniques have significantly contributed to elucidate various signaling networks, dynamics of protein-protein interactions and molecular understanding of disease mechanism. However, the global scale examination of a proteome is extremely challenging due to the unavailability of a single analytical platform capable of handling the diversity and extent of proteome complexity. For instance, if we want to investigate potential biomarker in plasma of patients with macular degeneration, the human biological fluid has few highly abundant proteins which make it difficult to identify the low abundant proteins present in the same sample. As a single analytical platform that is capable of analyzing a complete proteome map of cells or tissues is not available to date, multiple bioanalytical approaches coupled together can be used to achieve maximum coverage of entire proteome in a system. The combination of the approaches and their configuration depends on the samples, resources, time and desired outcome.

A typical workflow in mass spectrometry based proteomic analysis consists of three blocks: the separation techniques, the mass spectrometer, and the bioinformatics tools. Multi-dimensional chromatography and/or electrophoretic techniques are used to pre-fractionate highly complex proteomes, to reduce their complexity, followed by liquid chromatography-mass spectrometry/mass spectrometry (LC-MS/MS) analysis. The output from mass spectrometry is then analyzed using bioinformatics tools.

Age-related macular degeneration (AMD), as its name suggests, is a disease of the elderly. It is one of the leading causes of blindness in elderly in developed countries [1]. AMD is the degeneration of macula which is responsible for the central vision that enables us to drive and read. It can be broadly divided into two forms; dry AMD and wet AMD. Dry AMD affects a larger population, accounting for around $90 \%$ of total AMD. The disease is caused when drusen start accumulating in the macula between retinal 
pigment epithelium (RPE) and the Bruch's membrane. RPE is primarily responsible for clearing the damaged photoreceptors by phagocytosis. In the older population, the reduced capacity of RPE to clear the debris causing atrophy of the retina leads to the loss of vision. In contrast, wet AMD is caused due to the growth of the abnormal blood vessel in the macula causing vision loss.

There are few treatments available for wet AMD, but no FDA approved drug is available till date to treat dry AMD. Some of the FDA approved drugs for wet AMD and drugs that are under investigation are listed in Table 1-1. This has enticed the scientific community towards the understanding of the disease progression of dry AMD at the molecular level. Some studies have suggested that the inflammation and activation of the innate immune system are involved in the advancement of AMD. However, the molecular mechanism to explain the complex etiology of AMD including RPE cell death, damage due to oxidative stress, mitochondrial dysfunction and the accumulation of toxic components such as lipofuscin and other oxidative damaged product still needs to be interrogated to develop targeted therapies for AMD.

The retinal pigment epithelium (RPE) is one of the most important part of the human eye that affected due to AMD. RPE is a hexagonal, pigmented monolayer. It is tightly packed with the tight junction and adherens junction proteins and forms an integral part of the outer blood retinal barrier (BRB) (Figure 1-1A). The RPE layer in the human eye is located between the photoreceptor, which is prone to light induced oxidative stress, and the choroid that has a high supply of oxygen (Figure 1-1B) [2].

Oxidative stress has long been hypothesized to contribute to the pathogenesis and progression of AMD. The lipids in the RPE originate primarily from the photoreceptors that are phagocytosed and are eliminated via the choroid. When we age, the RPE deteriorates in its functions leading to the accumulation of the lipids like docosahexaenoic acid (DHA, a primary component of photoreceptor outer segments) in the Bruch's Membrane (BM). Over time, these are modified into harmful oxidized lipoprotein (oxLDL) products, a component of drusen [3]. It is believed that reduced capabilities to absorb light and to compensate for oxidative damage are important factors that initiate the chain of events leading to the onset of AMD in the older population $[2,4$, 5].

Oxidized lipoprotein formation within the eye is a complex process where both lipids and proteins of the LDL molecule are oxidized. The oxidation of LDL is supposed to be modified in a stepwise method. In the initial phase of modification, the lipid portion of the lipoprotein gets oxidized when they react with the oxidizing agents. This is followed by the reaction of lipid oxidation products with the apoB protein moiety of the LDL resulting in the oxidative change in the amino acid side chain forming the oxLDL[6]. Understanding the mechanisms for the fate of oxLDL within the RPE cells and the change in transcriptome and proteome of the RPE cells due to oxLDL would elucidate the molecular events in the pathogenesis of AMD. This would decipher the role of oxidative stress due to oxLDL to provide new opportunities for better treatment and prevention of the disease. 
Table 1-1. List of FDA approved/investigational drugs for dry and wet AMD.

\begin{tabular}{|c|c|c|c|c|}
\hline $\begin{array}{c}\text { Drug/Device } \\
\end{array}$ & Time & Dry vs Wet AMD & Company & Mechanism of Action \\
\hline Approved & & & & \\
\hline Implantable telescope & Jul-10 & End-stage AMD & $\begin{array}{l}\text { VisionCare } \\
\text { Ophthalmic } \\
\text { Technologies }\end{array}$ & $\begin{array}{l}\text { It magnifies the overall } \\
\text { image while reducing the } \\
\text { relative size of the central } \\
\text { blind spot }\end{array}$ \\
\hline $\begin{array}{l}\text { Lucentis(ranibizumab } \\
\text { injection) }\end{array}$ & Jun-06 & $\begin{array}{l}\text { Advanced or "wet" } \\
\text { form of macular } \\
\text { degeneration }\end{array}$ & $\begin{array}{l}\text { Genentech, in } \\
\text { collaboration } \\
\text { with Novartis } \\
\text { Ophthalmics }\end{array}$ & $\begin{array}{l}\text { It inhibits vascular } \\
\text { endothelial growth factor } \\
\text { (VEGF) }\end{array}$ \\
\hline $\begin{array}{l}\text { Macugen (pegaptanib } \\
\text { sodium injection) }\end{array}$ & & $\begin{array}{l}\text { Advanced or "wet" } \\
\text { form of macular } \\
\text { degeneration }\end{array}$ & $\begin{array}{l}\text { Eyetech Inc. } \\
\text { and Pfizer }\end{array}$ & $\begin{array}{l}\text { It contains pegaptanib ,a } \\
\text { selective vascular } \\
\text { endothelial growth factor } \\
\text { (VEGF) antagonist }\end{array}$ \\
\hline Eylea (aflibercept) & Nov-11 & $\begin{array}{l}\text { Advanced or "wet" } \\
\text { form of macular } \\
\text { degeneration }\end{array}$ & $\begin{array}{l}\text { Regeneron } \\
\text { Pharmaceuticals } \\
\text { and Bayer } \\
\text { HealthCare }\end{array}$ & $\begin{array}{l}\text { It is an anti-vascular } \\
\text { endothelial growth factor } \\
\text { (anti-VEGF) treatment }\end{array}$ \\
\hline $\begin{array}{l}\text { Visudyne drug } \\
\text { treatment } \\
\text { (Photodynamic } \\
\text { Therapy or PDT) }\end{array}$ & & \multicolumn{2}{|c|}{$\begin{array}{l}\text { Wet form of macular Novartis } \\
\text { degeneration }\end{array}$} & $\begin{array}{l}\text { It uses photodynamic } \\
\text { therapy (PDT) that destroys } \\
\text { abnormal blood vessels. }\end{array}$ \\
\hline $\begin{array}{l}\text { Laser treatment } \\
\text { Investigational }\end{array}$ & & \multicolumn{2}{|l|}{ Wet AMD } & photocoagulation \\
\hline $\begin{array}{l}\text { Eylea and antibody } \\
\text { combination therapy }\end{array}$ & & & \multicolumn{2}{|l|}{ Regeneron } \\
\hline $\begin{array}{l}\text { Avastin } \\
\text { (bevacizumab) }\end{array}$ & & & \multicolumn{2}{|l|}{ Genentech. } \\
\hline OHR-102. & Nov-15 & Wet AMD & \multicolumn{2}{|l|}{$\begin{array}{l}\text { Ohr } \\
\text { Pharmaceutical. }\end{array}$} \\
\hline MC-1101 & $\begin{array}{l}\text { 2012, phase } 3 \\
\text { testing of the } \\
\text { drug therapy }\end{array}$ & $\begin{array}{l}\text { Prevent dry AMD to } \\
\text { progressing to wet } \\
\text { AMD }\end{array}$ & MacuCLEAR & $\begin{array}{l}\text { increases blood flow to the } \\
\text { choroid }\end{array}$ \\
\hline \multicolumn{5}{|l|}{ Drug Implants } \\
\hline Ranibizumab PDS & 2012 & Wet AMD & Genentech & $\begin{array}{l}\text { It inhibits vascular } \\
\text { endothelial growth factor } \\
\text { (VEGF) }\end{array}$ \\
\hline Renexus (NT-501) & & $\begin{array}{l}\text { Retinal } \\
\text { Degenerative } \\
\text { Disease }\end{array}$ & $\begin{array}{l}\text { Neurotech } \\
\text { Pharmaceuticals }\end{array}$ & $\begin{array}{l}\text { Implant that contains } \\
\text { genetically modified human } \\
\text { cells capable of secreting a } \\
\text { nerve growth factor that } \\
\text { rescues and protect dying } \\
\text { photoreceptors in the retina }\end{array}$ \\
\hline
\end{tabular}


Table 1-1. (Continued.)

\begin{tabular}{|c|c|c|c|c|}
\hline Drug/Device & Time & Dry vs Wet AMD & Company & Mechanism of Action \\
\hline \multicolumn{5}{|l|}{ Artificial Retinas } \\
\hline Artificial Silicon & & & Optobionics & It is a microchip that \\
\hline Retina (ASR) & & & & $\begin{array}{l}\text { stimulates healthy retinal } \\
\text { cells to restore vision }\end{array}$ \\
\hline \multicolumn{5}{|l|}{ Stem Cells } \\
\hline & Jun-15 & Dry AMD & Ocata & \\
\hline & & & Therapeutics/ & \\
\hline & & & Astellas & \\
\hline & & & Institute for & \\
\hline & & & Regenerative & \\
\hline & & & Medicine & \\
\hline & & & $(\mathrm{AIRM})$ & \\
\hline & $\begin{array}{l}\text { June } 2015 / \\
\text { phase } 1 / 2 \\
\text { clinical trial }\end{array}$ & Dry AMD & StemCells Inc & \\
\hline
\end{tabular}

Sources: https://www.allaboutvision.com/conditions/amd-treatments.htm, https://www.eylea.us/?WHGRedir=1, http://www.bausch.com/ecp/Our-Products/RxPharmaceuticals/Rx-Pharmaceuticals/Macugen-pegaptanib-sodium-injection, and https://www.pdr.net/drug-summary/visudyne?druglabelid=651 


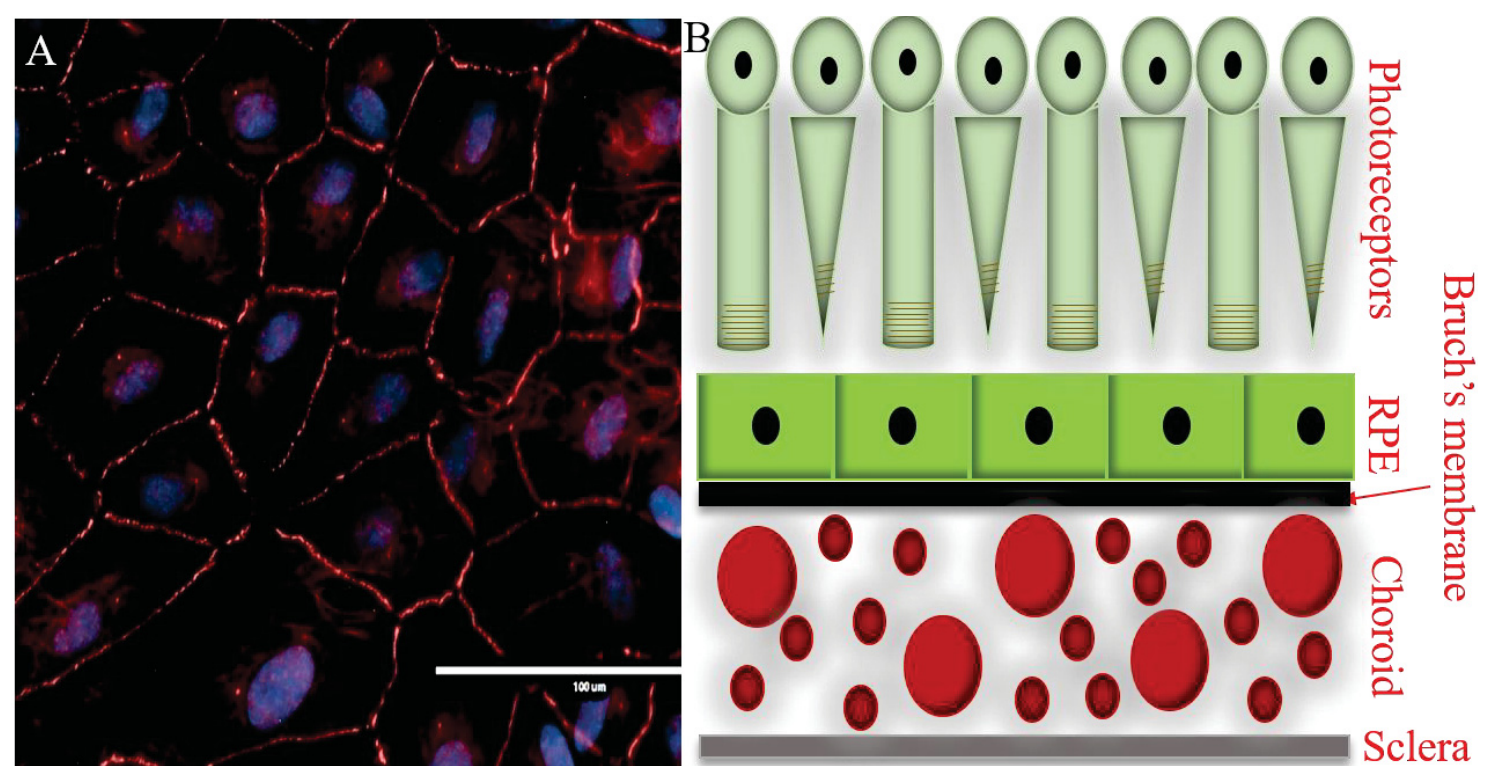

Figure 1-1. RPE structure and location.

(A) Hexagonal structure of cultured human retinal pigment epithelial cells (ARPE-19) shown by ZO-1(Zonula occludens-1) staining. (B) The location of RPE in the context of an eye. 
As AMD is one of the leading causes of blindness in elderly people and with the lack of the complete knowledge on disease progression, it will continue to affect a large population. The facts that there is no available treatment for dry AMD and that the treatments for wet AMD are not showing the desired outcomes, there is an immense and immediate need of understanding the molecular mechanism of the pathogenesis and progression of the disease. The differential proteomic analyses of the eye affected with AMD and a normal eye will definitely provide a great insight into the protein expression patterns. These lead to a path towards the discovery of a drug to treat, cure or reverse the disease and helps to improve the quality of life of patients living with AMD.

\section{Dissertation Hypothesis and Specific Aims}

The research presented in this dissertation encompasses two major components. (1) The first component is the bioanalytical component that focuses on evaluation of proteomics workflows centered around mass spectrometry as the key technology. As stated in the following statement by J. Van Eyk, "the diversity and extent of proteome complexity will not and cannot be solved by a single technology", global scale examination of proteome is extremely challenging. Hence, a reliable and robust analytical method to study the proteome of a biological system needs to be established which can eventually provide a foundation to study the proteome perturbation due to oxLDL treatment in the ARPE-19 cells. The hypothesis for this component is that fractionation of the proteome at the protein or peptide level prior to LC-MS/MS analysis increases the number of identified proteins and enhances access to low abundance proteins. (2) The second component of my research focuses on application of global-scale and targeted approaches to examination of the molecular response of retinal pigment epithelium (RPE) cells to oxidized lipoproteins. The central hypothesis for this component is that oxidized low density lipoprotein (oxLDL) is internalized by the RPE cells through the action of scavenger receptors CD36 and CD5L/AIM, and induces a cytoprotective molecular response in the RPE cells.

\section{Aim 1. To Evaluate the Capabilities of Bioanalytical Platforms in Terms of Extent and Depth of Proteome Coverage}

In recent years, major advancements in the area of mass spectrometry have contributed to a shift from use of proteomics workflows that were based on 2D gel electrophoresis to alternative workflows that incorporate powerful tandem mass spectrometry technologies, often in combination with up-stream fractionation at the protein and/or peptide level. With any proteomic technique, there is a bias towards more abundant proteins. Multiple analytical techniques are required to study the entire proteome of a biological system which depends upon the desired outcome, sample, resources and time. Three bioanalytical platforms will be analyzed to characterize mitochondrial proteome. The main objective of this aim is to evaluate the capabilities of bioanalytical platforms regarding the extent and depth of proteome coverage. 


\section{Aim 2. To Determine the Roles of Scavenger Receptors CD36 and CD5L/AIM in the Clearance of OxLDL by the Human Retinal Pigment Epithelial Cells}

Accumulation of oxidized lipoproteins (oxLDL) in the eye is considered as an important trigger in the pathogenesis of AMD. OxLDL are identified by special receptors in cells known as scavenger receptors. To better understand the role of two scavenger receptors in clearance of oxLDL in the eye we postulated following hypothesis based upon our central hypothesis: i) we hypothesize that the transmembrane scavenger receptor CD36 binds oxLDL on the surface of RPE cells and mediates oxLDL uptake by the cells; ii) we hypothesize that the RPE cells express and secrete the soluble scavenger receptor CD5L/AIM. We further postulate that CD5L/AIM present in the extracellular

space binds oxLDL and facilitates its clearance by the RPE cells. As an in vitro model of the RPE, we will use the well-established ARPE-19 cell line. We will investigate the role of CD36 and CD5L/AIM involvement in uptake of oxLDL by ARPE-19 cells and the molecular effect of this uptake.

\section{Aim 3. To Characterize OxLDL-induced Molecular Response in the RPE Cells}

Our working hypothesis is that the uptake of oxLDL by the ARPE-19 cells induces a cytoprotective molecular response. We postulate that this molecular response will be multifaceted and involve alterations of diverse pathways and networks. To fully capture this response, we will use global-scale, discovery-based approaches to characterize oxLDL-induced alterations in the ARPE-19 transcriptome and proteome

\section{Dissertation Overview}

In chapter 2 of my dissertation, I investigated on the aim 1 i.e. bioanalytical component of my dissertation. The main objective of this aim was to evaluate the capabilities of three different bioanalytical platforms in terms of extent and depth of proteome coverage. When performed before LC-MS/MS analysis, it provided better extent and in-depth proteome coverage. In chapter 3, I investigated aim 2 i.e. the scavenger receptors that are involved in the transport of oxLDL. I described the effect of oxLDL treatment and the mechanistic understanding gained from the results after the treatment on ARPE-19 cells. In chapter 4 and 5, I explored, aim 3 of my dissertation. In chapter 4, I examined the transcriptome alteration in ARPE-19 cells due to oxLDL and LDL treatments. The main objective of this study was to understand oxLDL induced alterations in various mechanistic pathways potentially related to AMD pathogenesis. In chapter 5, I assessed the proteomic alteration in ARPE-19 cells after oxLDL and LDL treatments and discussed a new bioanalytical platform used in this study. 


\section{CHAPTER 2. PROTEOMICS IN BIOLOGICAL SYSTEM: STUDY OF BIOANALYTICAL PLATFORMS FOR BETTER PROTEOME COVERAGE ${ }^{1}$}

\section{Introduction}

Proteomics, as coined by Marc Wilkins and colleagues during the early 1990s, is the large-scale study of the proteome, i.e. a set of proteins present in a biological system under a given set of conditions [7]. The accomplishments of the human genome project that annotated the human genome have steered the identification of over twenty thousand protein coding genes [8]. Besides the genetic instructions encoded in the genome, the knowledge of the proteins involved is equally vital to understand the biological processes that include synthetic, catalytic and regulatory functions occurring inside a living cell [9]. Proteins are the fundamental players in nearly all the biological processes that are concurrently active in every living cells working in a very coordinated fashion [10].

The analysis of proteins in a particular system is difficult due to unavailability of tools that can efficiently analyze the whole proteins in biological systems, unlike the gene expression measurement which has a long history of well characterized tools [11]. Many proteins, produced by specific genes, undergo different posttranslational modifications. In addition, the variety and extent of posttranslational modifications of these proteins vary due to different environmental conditions within and outside the cells [12]. Thus, elucidating the structure and function of proteins in a proteome along with the understanding of their dynamic interaction with other inter- and intracellular proteins and components are the major challenges in protein biology [13].

\section{Mass Spectrometry Based Proteomics}

Proteomics is a widely used strategy in the field of biology. Proteomic approaches are applied for proteome profiling, identification and localizing of posttranslational modification within the protein, and the study of protein-protein interactions [14]. There are various techniques that can analyze different aspects of protein structure and function such as protein arrays, analytical microarrays, functional protein microarrays, highthroughput protein hybridization and crystallization, analytical and reverse phase microarrays [15]. However, mass spectrometry has emerged as the most prominent strategy used at present for global-scale analysis of proteins in complex biological systems. The early methods for protein sequencing included Edman degradation in which proteins were sequenced by chemically cleaving the amino acids one at a time from the N-terminus. It required a high level of expertise, and therefore it could not be applied to all the protein sequencing, for example, if the N-terminus were chemically modified, this process would not work [9]. By late-1990, the Edman degradation technique was

\footnotetext{
${ }^{1}$ Reprinted with open access permission. Giorgianni, F. Koirala D, Weber T. Giorgianni-Beranova S., Proteome analysis of subsarcolemmal cardiomyocyte mitochondria: a comparison of different analytical platforms. Int J Mol Sci, 2014. 15(6): p. 9285-301.(19)
} 
gradually replaced by mass spectrometry based strategies. In the last two decades, mass spectrometry based techniques have profoundly influenced proteomics research. They have become dominant analytical techniques not only in large-scale 'proteomic' studies but also in mechanistic and hypothesis driven studies, to successfully identify and quantify a single component of a proteome [16-18].

Global scale examination of the proteome is still extremely challenging, as a single protein encoded by a gene may have multiple isoforms with different functions. Moreover, the protein may be modified by post-translational modifications or may be involved in interactions with other proteins within the cell. In addition, the mammalian cells, tissues, and body fluids display a complex mixture of a wide range of protein concentrations. Therefore, no single analytical platform is capable of solving the diversity and extent of proteome complexity [19]. Even with the development of various separation techniques, the advancement in mass spectrometers, and the improvement of computational bio-informatics tools, many important analytical needs still remain to be fulfilled. Among such limitations is the ability to analyze complex biological mixture and to identify and quantify low abundant proteins present in complex protein mixtures. These issues can be addressed by effective separation techniques, state-of-art mass spectrometry instruments and efficient data processing and analyzing computational tools [14].

Based on the characterization of peptides or proteins by mass spectrometry, the field of proteomics can be divided, in general, into two main analytical approaches: topdown and bottom-up. The top-down proteomic approach focuses on the analysis of intact proteins without proteolytic digestion [20]. In the bottom-up proteomic approach, on the other hand, proteins in a sample are first digested by a proteolytic enzyme to generate peptides. Analytical data diagnostic of the peptide sequences are then obtained by mass spectrometry, commonly after fractionation of the peptide mixture by liquid chromatography. The sequence-diagnostic information obtained from the mass spectrometer for the $\mathrm{m} / \mathrm{z}$ of the peptides and their respective fragments are then compared to the in-silico data from a protein sequence database to identify the protein [21]. Bottomup proteomics is at present the most widely used approach.

\section{Components of Mass Spectrometry Based Bioanalytical Proteomic Workflow}

In general, typical bioanalytical workflows for bottom-up proteomics comprise of three main components (Figure 2-1): i) Sample preparation ii) Protein and/or peptide separation and iii) Protein identification/quantification, interpretation and data analysis. There are many variations within the elements of this generic workflow.

Sample preparation. To study the proteome in a given biological sample, the first and critical step is the extraction of proteins. For the proteomic analysis, the objective is to recover as much protein as possible and with very little contamination with 


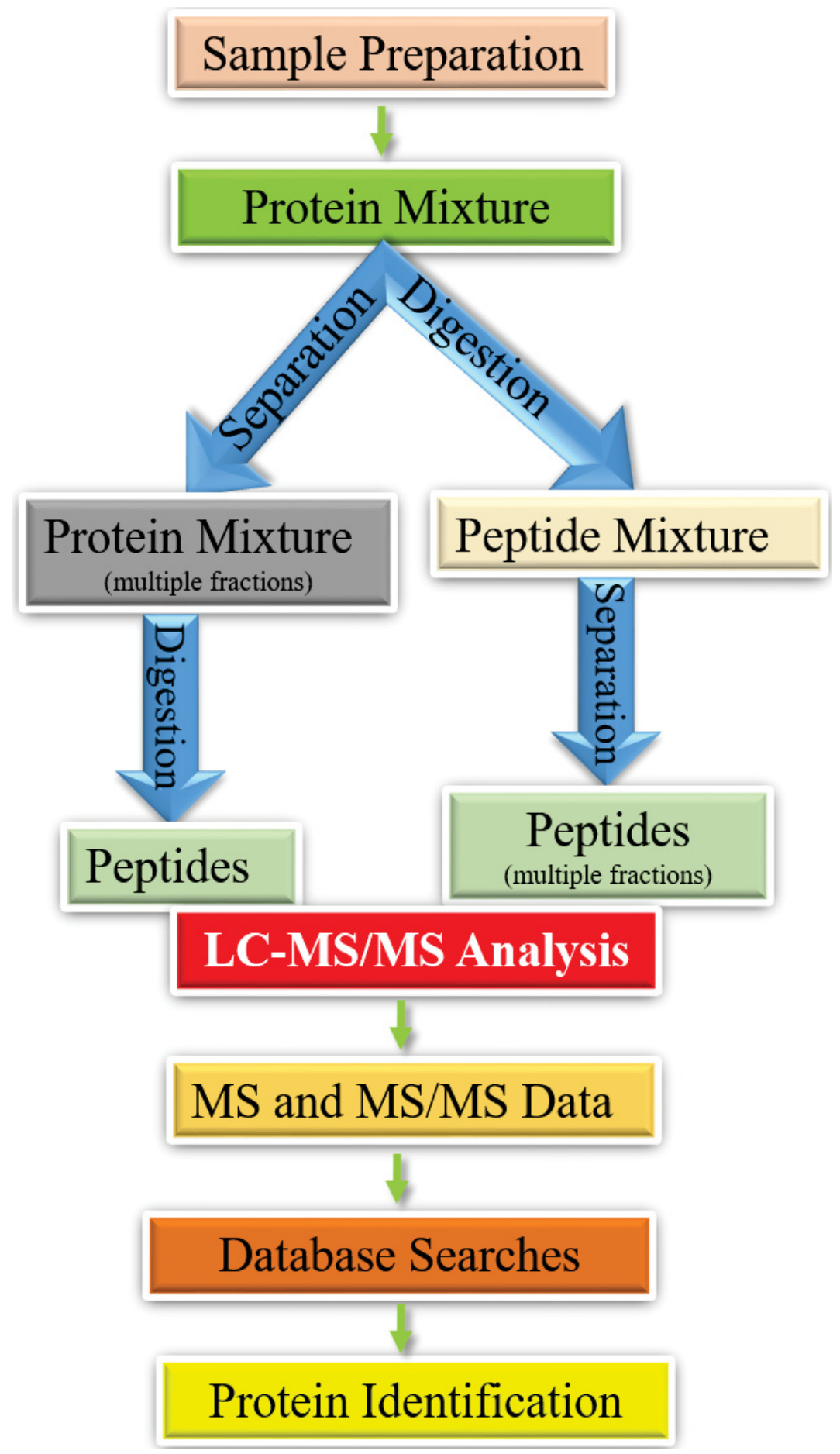

Figure 2-1. General scheme for proteomic analysis. 
the other biological materials such as lipids and nucleic acid [22]. Sample preparation techniques differ depending upon the starting sample material and purpose of the experiment. In general, for cells and tissues, the samples are first homogenized, sonicated, pulverized or disrupted. In contrast, for biological fluids, the samples are first depleted [23]. The protein can then be extracted with the aid of detergents (SDS, CHAPS, cholate, Tween), reducing agents (DTT, mercaptoethanol, thiourea), denaturing agents and enzymes. Protease inhibitors are commonly used to prevent proteolytic protein digestion. A number of methods have been developed by using the above mentioned agents in different combinations. However, care should be taken if some of the above mentioned agents interfere with the downstream proteomic analysis by a mass spectrometer.

Protein/peptide separation. Since the analysis of complex protein mixture is difficult, it is very important to consider separation of the proteins and/or peptides before mass spectrometry analysis. Separation of a complex protein and/or peptide mixture can be done by either gel-based or gel-free methods, and various separation methods have been incorporated in proteomics workflows. Selected methods used for this purpose are discussed in more detail below.

Separation at protein level: Gel electrophoresis. Gel electrophoresis is widely used in proteomics to separate proteins. After the rise of proteomics in the 1990s, twodimensional (2D) gel electrophoresis had been an integral component of most original proteomics workflows, and a method of choice for relative protein quantification in differential protein expression studies [24]. Advancement of powerful tandem mass spectrometry instrumentation and associated bioinformatics tools caused a departure from 2D gel electrophoresis-based workflows in recent years. In two-dimensional gel electrophoresis, the proteins first undergo separation by isoelectric focusing (IEF) based on the net charge of the protein and then the charge-separated proteins are separated in the second dimension according to their molecular weight by SDS-PAGE [25]. 2D gel electrophoresis has a very high resolving power as thousands of proteins can be separated in a single gel. 2D separation provides high information content on each separated protein as each protein is characterized by its $\mathrm{pI}$ and molecular weight and when analyzed by mass spectrometry after 2D separation protein identity can be known. This method can also effectively separate individual modified protein forms which are very important for identifying the posttranslational modification of proteins [25]. However, this technique is very labor intensive as it requires a lot of time and effort to gel fractionation, protein extraction and peptide digestion [26]. Also, the sensitivity of 2D gel electrophoresis depends upon staining method [27], and only a fraction of proteome can be revealed in a single 2D gel [14].

Besides SDS-PAGE based protein separation, an analogous strategy is the onedimensional gel-based proteomic strategy that uses in-gel IEF separation at protein level combined with down-stream LC-MS/MS. This workflow was first introduced by our laboratory and applied analyze the proteome in the human pituitary and human prostate cancer tissue $[32,33]$. This method has also been successfully used for phosphoproteome discovery in the pituitary and prostate [33-35]. Iso-electric focusing is a high resolution 
separation technique which separates proteins according to their isoelectric points ( $\mathrm{pI}$ ) within a continuous $\mathrm{pH}$ gradient. Immobilized $\mathrm{pH}$ gradient (IPG) strips are commercially available and are used to separate the proteins according to their pI. The IPG strips with wide $\mathrm{pH}$ gradient $\mathrm{pH}$ 3-10 are often used for whole proteome analysis [36]. Proteins are amphoteric in nature which means that they contain both acidic and basic groups and carry a charge depending on the $\mathrm{pH}$ of the surroundings. Since proteins are negatively charged in solution at $\mathrm{pH}$ above the $\mathrm{pI}$ and positively charged below the $\mathrm{pI}$, a protein, during electrophoresis, will migrate towards cathode if it has positive net charge in a $\mathrm{pH}$ region below its $\mathrm{pI}$. Similarly, at $\mathrm{pH}$ values above its $\mathrm{pI}$, the negatively charged protein molecules will move towards the anode. Thus the protein molecules move in the electric field until they reach the $\mathrm{pH}$ region that corresponds to the protein $\mathrm{pI}$, where they focus into sharp bands. For comprehensive proteome analysis, following IEF separation, the entire IPG strip is sectioned and the proteins in each section are digested. The peptide digests from each section are subjected to LC-MS/MS to obtain sequence-determining data for peptide and protein identification.

Separation at peptide level: Gel free separation. A collection of gel free strategies for separation of complex mixtures in proteomics has been developed, which utilize a combination of liquid chromatography methods with different chemistries. Among these gel free methods, multidimensional protein identification technology (MudPIT) is a very popular two dimensional liquid chromatography (2D-LC) analytical workflow which is a widely accepted method for analysis of proteomes from a wide variety of samples [37]. MudPIT is also known as shot gun proteomics. In MudPIT, a whole proteome is first digested, and the highly complex peptide mixture is loaded directly into a microcapillary column packed with strong cation exchange (SCX) in the first dimension which is coupled to reverse phase (RP) column in the second dimension. The coupling of SCX and RP can be either offline or online, with the second dimension (RP) comprising the LC component of LC-MS/MS. The two dimensional separation gives better coverage of identified proteins than 2D gel electrophoresis method due to the difference in selectivity of peptides by SCX and RP. In addition, there is good compatibility between the SCX mobile phase and second dimensional RP separation. However, like any other methods, MudPIT has some disadvantages. For instance, the proteins are digested before separation makes the identification of isoforms difficult [3840].

An alternative method for MudPIT has been developed that utilizes the RP column in both the dimensions is known as reverse phase reverse phase separation (RPRP) method. In a study that explored the RP column for separation of peptides in both dimensions found that the mobile phages had a significant impact in RP selectivity and selection. This study showed that the use of mobile phases with different $\mathrm{pH}$, particularly $\mathrm{pH} 10$ on the first dimension and $\mathrm{pH} 2.6$ on the second dimension, for a system with $\mathrm{C}_{18}$ column provided the optimum orthogonality [41]. The RP-RP method permitted the collection of multiple fractions with minimal overlap in the first separation resulting no peptide losses as well as better recovery of both long and short peptides [42]. Various studies have however demonstrated the better performance of PLRP-based approaches in 2D LC-MS/MS applications for shotgun proteomic studies compared to the MudPIT 
platform. PLRP based approach is a slight modification in the RP-RP method, using polymer-based reversed-phase (PLRP) column and high-pH mobile phase is used in the first dimension, followed by conventional $\mathrm{C} 18$ reversed-phase separation at low $\mathrm{pH}[43$, 44].

Liquid chromatography and mass spectrometry. The complex mixture of peptides in bottom-up proteomics workflows that are obtained either by fractionation or without fractionation are separated by RP liquid chromatography before MS and MS/MS analysis. The use of Ultra High Performance Liquid Chromatography (UPLC) as an analytical technique for the separation of peptides has drastically increased the efficiency. As a result, it is an integral part of peptide/protein identification by mass spectrometry. In recent years, HPLC/UPLC has experienced substantial developments and new separation combinations of different stationary phases or the use of various mobile phases.

However, the reversed phase chromatography (RPC) is one of the widely used techniques for the separation of proteins and peptides. The separation of tryptically digested peptides in proteomics research using RPC is based on the interaction of sample analytes with the hydrophobic stationary phase. Nano-LC is another very important and most commonly used tool for separating the peptides/proteins before mass spectrometry analysis. The main advantage of using the nano capillary LC is to enhance the sensitivity caused by the reduced radial dilution in a narrow column [45]. The use of nano-UPLC as a novel analytical technique is capable of using chromatography resins with diameter less than $2 \mu \mathrm{m}$. It can, therefore, overcome problems regarding high pressure, improving the resolution, sensitivity and speed.

Mass spectrometry is a powerful analytical technique used to identify and quantify unknown compounds, biomolecules, proteins, and peptides. It measures the ratio between the mass and charge of ions which gives various information on proteins regarding their identity, chemical modification, and structure. Recent advances in the field of mass spectrometry have been one of the major driving force in the field of proteomics. Mass spectrometer mainly consists of three different parts: (i) ion source (ii) mass analyzer and (iii) detector. The ion source converts analytes into gas phase ions as they must be charged in the gas phase to be analyzed by mass spectrometer. The charged ions are then separated by the analyzer on the basis of their mass-to-charge ratio. Finally, the ions resolved by the analyzer are detected by a detector which will amplify the response to give a measurable response [46].

The Nobel Prize awarded to two scientists Dr. John Fenn and Dr. Koichi Tanaka in 2002 for the development of two ionization techniques signifies the importance of ionization in mass spectrometry based techniques. The development of two ionization techniques MALDI (matrix-assisted laser desorption ionization) and ESI (electrospray ionization) revolutionized the protein identification by mass spectrometry. These ionization methods allowed the vaporization and ionization of large nonvolatile proteins and peptides molecules which were not achieved by the earlier ionization methods such as EI (Electron impact) and CI (Chemical ionization)[47]. 
MALDI ionization method uses laser energy absorbing matrix which creates ions from large molecules that usually produces singly charged ions[48]. The protein samples are mixed with an appropriate solvent and are allowed to crystallize. So when this mixture is irradiated by laser energy, the analyte in gas phase is carried into the mass spectrometer. MALDI, however, has few drawbacks. It has a high preference to ionize basic peptide as it preferentially ionizes arginine containing peptide in a tryptic peptide mix. Due to the same reason, it poorly ionizes the phosphopeptides as the phosphate group possesses acidic properties [49].

The electrospray ionization is another most widely used technique for ionization. The liquid sample solution is directly converted into gas phase ionized molecules in the presence of a strong electric field which aids on-line coupling of HPLC with ESI based mass spectrometer [50]. This results in multiple charged analyte ions which are desirable for proteomics as the higher molecular weight compounds could be easily analyzed by a mass spectrometer with low mass range. One of the main advantages of ESI is that it produces multiple charged molecules/ions and can be easily coupled with HPLC [51].

Along with the development of ionization techniques, improvements in mass analyzers also aided the revolution of mass spectrometry based proteomics. Mass analyzer measures gas phase ions with respect to their mass-to-charge ratio (m/z). The popular mass analyzers in the field of proteomics are linear ion trap, orbitrap, and QTOF.

Linear ion trap mass spectrometer stand alone has been for a number of years the prominent configuration and workhorse of global proteomics [52]. The linear ion trap mass analyzer consists of a set of quadrupole rods that confines ions radially with radio frequency $(\mathrm{RF})$ and a static electric potential on end to confine the ions axially. It can trap ions with different $\mathrm{m} / \mathrm{z}$ values over a period and eject sequentially into the detector. Ion traps collect, store and eject the ions and these activities cycle through many rounds. In each round, ion traps can isolate and fragment peptide ions. In the field of proteomics, linear ion traps, quadrupoles, orbitraps, and time of flight (TOF) are the commonly used mass analyzers. Each analyzer has its own specific characteristics. These analyzers either alone or in combination as a component of hybrid mass analyzers are being continuously developed or improved for better proteomic applications.

The analytes are acquired in different ways by a mass spectrometer. The datadependent acquisition (DDA) is one of the preferred methods for discovery-based proteomics where each full MS scan is followed by a specific number of MS/MS scans. Since its inception around the 1990s, the DDA continues to dominate the field of proteomics as a preferred method for deciphering the information from a complex mixture of peptides. With the complex samples (i.e., complex peptide mixture) a large number of compounds elute from the nano-LC column in a given instant. The DDA method first acquires the full spectrum of peptides (the MS spectra) of the ions that coelute from LC [10]. The mass spectrometer then selects a pre-defined precursor in real time (usually the most abundant one). It then acquires the fragment ion-spectra for the selected precursor ions. The mass spectrometer instrument runs in iterative acquisition cycle first to select the precursor ion spectra, i.e. (MS or MS1), and then the fragment ion 
spectra (MS/MS or MS2). The instrument then puts a mass into an exclusion list (Dynamic exclusion principle) after its MS/MS scan is acquired so that the peptides that co-elute with high abundant peptides have chances of detection by the mass spectrometer. The instrument hence selects the most abundant ions for further MS/MS scans and hence the analytes that show the strongest signals have highest chance to be successfully identified. Although DDA has been used as an ultimate data acquisition method in the field of proteomics, it only samples peptides with restricted dynamic range. This is because the precursor ion selection method in the DDA is designed in such a way that it only selects the most abundant ions [53]. An example of DDA by LTQ ion trap mass spectrometer is shown in Figure 2-2.

Another emerging data acquisition method in the field of proteomics is data independent acquisition (DIA) where there is no real-time selection of precursor ions based upon their abundance [54]. In DIA method, entire range of the precursor ions along with their MS/MS signals are acquired. The method allows the entire range of possible precursor ions mass to be analyzed that elutes from the nano-LC separation. Thus a mixture of several precursor ion fragments that co-elute and MS/MS spectra of the fragment ions from different peptides that co-dissociate irrespective of their abundance are acquired. This method offers a major advantage over the DDA method in identifying low abundance peptides that do not have significant precursor peak [55].

Bioinformatics. Bioinformatics tools provide protein scientists the ability to analyze thousands of MS scans that are generated from a mass spectrometer. The proteins are extracted from samples and enzymatically digested to generate peptides. These peptides can then be detected by mass spectrometer by tandem mass spectrometry and appropriate database search algorithms. In tandem mass spectrometry, the precursor ions generated from the source are further fragmented by colliding with an inert gas such as helium or nitrogen that produce product ions. The masses of the product ions then can be matched with in-silico fragment of parent ions and assigned various correlation and scoring factors based upon how close the obtained spectra match the theoretical spectra. The proteins are then identified by the software by the matched product ions which are selected based upon various scores and filters that come with the software along with the mass spectrometer that we use. The significance of the identified peptides is calculated by FDR scores. A large set of protein sequence database is available for proteome searches, among which the most commonly used are Swiss-prot (Uniprot), and NCBI. After the proteins are identified using the protein search databases and the tools, other bioinformatics tools such as PANTHER [56], ClueGO [57], IPA [58], and STRING [59] are needed for functional characterization of identified samples. These online tools provide the functional interpretation of the identified proteins, and thus biological significance can be predicted.

In the study reported here, three different multidimensional bioanalytical platforms were used to analyze the proteome in mitochondria. The main objective of the study was to evaluate the capabilities of these platforms in terms of the extent and the depth of proteome coverage. The three platforms incorporated different first-dimension 

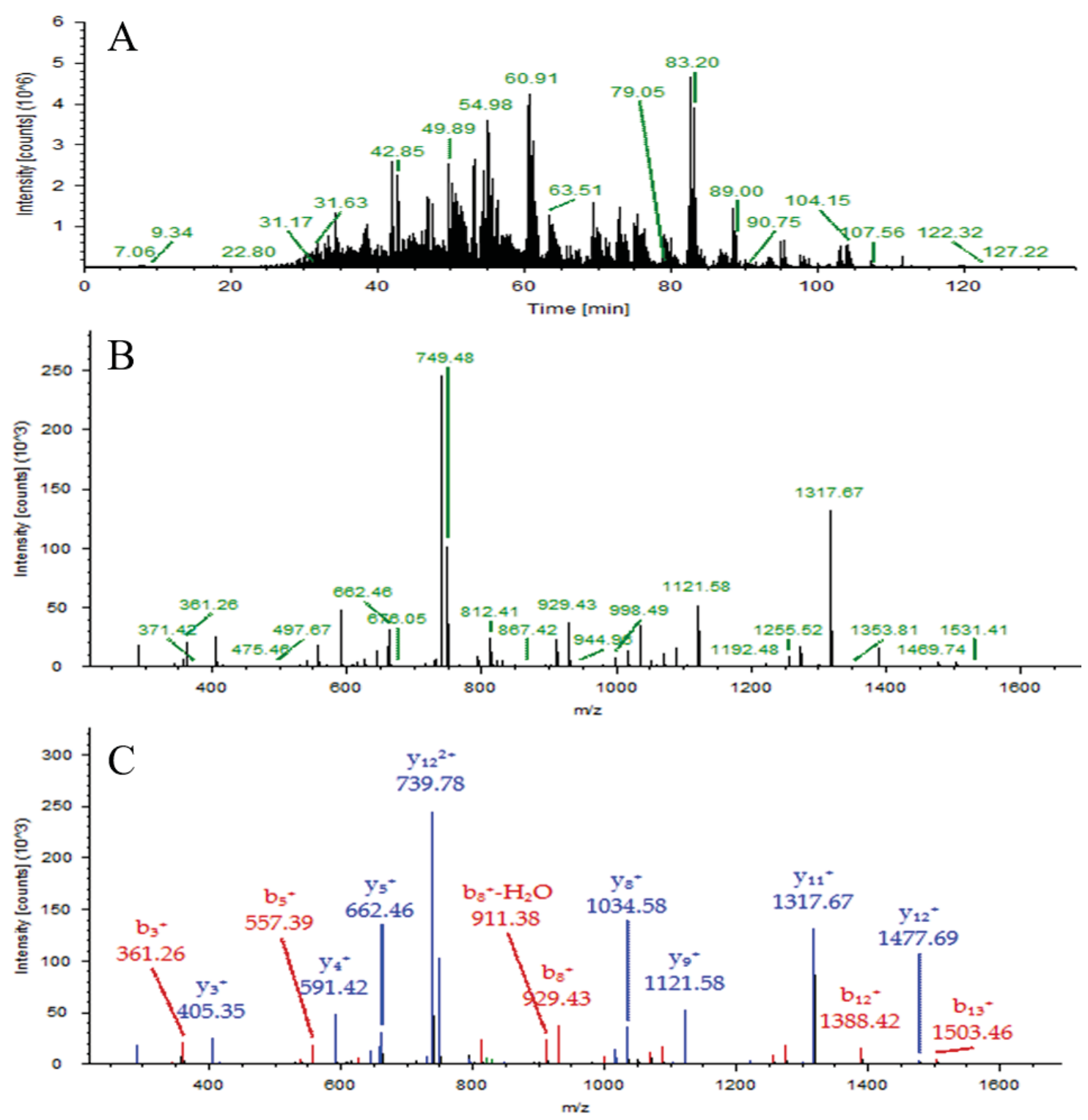

Figure 2-2. An example of tandem MS/MS in a data dependent acquisition setting.

(A) Chromatogram of LC, (B) MS-Spectrum of analytes eluting at the LC retention time around $83.99 \mathrm{~min},(\mathbf{C}) \mathrm{MS} / \mathrm{MS}$ measurement of precursor ion with $\mathrm{m} / \mathrm{z} 840$. In data dependent acquisition the mass spectrometer first measures full MS scan is followed by a specific number of MS/MS scans. The above example shows Peptides dissociate in a particular fashion, and MS/MS spectrum of a peptide ion with $\mathrm{m} / \mathrm{z} 840$ shows a series of $\mathrm{y}$ and $\mathrm{b}$ ions produced due to fragmentation. 
separation methods-PLRP (separation at the peptide level), in-gel IEF (separation at the protein level) and SDS-PAGE (separation at the protein level) - in combination with down-stream RP LC-MS/MS. Our investigation showed the superior performance of the SDS-PAGE-based platform. Survey of the mitochondrial proteome with this platform provided access to the highest number of mitochondrial proteins over a wide range of abundance levels. The mitochondria proteome profile that was obtained encompasses proteins with diverse functional characteristics. Thus, among the configurations evaluated, the SDS-PAGE-based platform was identified as the best choice as a multidimensional bioanalytical strategy for qualitative and quantitative mitochondrial proteome profiling.

\section{Materials and Methods}

\section{Protein Extraction}

The protein samples for the study were obtained from 8 week old SpragueDawley rats $(n=6)$ which were approved by our Institutional Animal Care and Use Committee. The subsarcolemmal population of mitochondria was isolated by homogenization of freshly collected heart tissue samples followed by differential centrifugation. Interfibrillar mitochondria (IFM) was discarded after 1500xg, and the subsarcolemmal (SSM) mitochondria were collected after 10000xg centrifugation. To confirm the purity of isolated mitochondria, the mitochondrial preparation was stained with Mito Tracker Red which is a mitochondria-specific dye and analyzed by flow cytometry, as previously described [60].

The individual $(n=6)$ mitochondrial preparation were pooled. The protein mixture was then mixed with buffer containing $400 \mathrm{mM}$ of ABC (ammonium bicarbonate), $8 \mathrm{M}$ urea and 1\% RapiGest and sonicated with sonicator probe four times for 30 seconds every 15 min intervals to extract proteins. The total protein concentration of the extracted proteins was quantified with the 2D-Quant protein assay kit (GE Healthcare, Piscataway, NJ, USA). The protein extract was divided into three equal portions and stored at $-80 \mathrm{C}$ until further processing. For each bioanalytical platform $400 \mu \mathrm{g}$ of protein was used, and $130 \mu \mathrm{g}$ were used in each technical replicates, i.e., $\mathrm{n}=3$ per platform.

\section{Isoelectric Focusing}

For isoelectric focusing the mitochondrial proteins were first dissolved in an IEF rehydration buffer consisting of urea $(7 \mathrm{M})$, thio urea $(2 \mathrm{M}), 3-[(3-$ cholamidopropyl)dimethylammonio]-1-propanesulfonate (CHAPS) detergent $(2 \% \mathrm{w} / \mathrm{v})$, immobilized $\mathrm{pH}$ gradient (IPG) buffer (carrier ampholyte mixture; $2 \% \mathrm{v} / \mathrm{v}$ ) and DTT $(0.3 \% \mathrm{w} / \mathrm{v})$. The IPG strips used for protein separation were $18 \mathrm{~cm}$ long and had nonlinear 3-10 $\mathrm{pH}$ gradient. $130 \mu \mathrm{g}$ of protein sample per strip was applied via passive IPG strip rehydration. Following parameters were used for focusing the protein samples: 
0-100 V gradient for $1 \mathrm{~min}$; $100 \mathrm{~V}$ fixed for 120 minutes; $100-500 \mathrm{~V}$ gradient over $1 \mathrm{~min}$; 500-3000 V gradient over 90 min and $3500 \mathrm{~V}$ constant for 8 hours. After the protein separation, the IEF strip was fixed with trichloroacetic acid $(20 \% \mathrm{w} / \mathrm{v})$ and with methanol: water: acetic acid (50:40:10 v: v: v). The strip was then divided into 15 sections of equal size.

\section{SDS-PAGE}

Criterion XT 4\%- 12\% Bis-Tris gels (13.3 x $8.7 \mathrm{~cm})$ from Bio-Rad was used for the SDS-PAGE separation. As mentioned earlier, $130 \mu \mathrm{g}$ of protein was used in each replicate ( $\mathrm{n}=3$, technical replicate). $130 \mathrm{ug}$ of protein sample was separated on 3 lanes (approximately $40 \mathrm{ug}$ per lane) at $200 \mathrm{~V}$ for 60 minutes. The separated lanes were stained with Coomassie Blue stain and then divided into 15 sections of equal size. The $8 \mathrm{~cm}$ gel was equally divided into 15 bands horizontally and cut into pieces manually and stored at $-20{ }^{\circ} \mathrm{C}$ after image acquisition.

\section{In-gel Protein Digestion}

Each section of the SDS PAGE was in-gel digested using the following protocol. The gel pieces were destained three times in 700uL of water/methanol solution (50:50, $\mathrm{v} / \mathrm{v})$ with $25 \mathrm{mM}$ ammonium bicarbonate buffer $(\mathrm{ABC})(\mathrm{pH} 8.0)$, changing the solution every $10 \mathrm{~min}$. The gel pieces were then washed with $1 \mathrm{~mL}$ of an acetic solution (acetic $\mathrm{acid} / \mathrm{methanol} / \mathrm{water}, 10: 40: 50, \mathrm{v} / \mathrm{v} / \mathrm{v}$ ) for $3 \mathrm{~h}$, changing the solution every $1 \mathrm{~h}$. Then the gels were soaked in water for 20 minutes followed by $200 \mathrm{nM} \mathrm{ABC}$ for next 20 minutes. The protein in IEF trip and processes SDS PAGE gel pieces were then reduced by $50 \mathrm{mM}$ DTT (in ABC) and alkylated with $110 \mathrm{mM}$ Idoacetamide and dried in a SpeedVac for 30 min. after dehydration with ACN. Then $150 \mathrm{ml}$ of Trypsin, $1.09 \mathrm{ug} / \mathrm{uL}$ of sequence grade modified trypsin (Promega) was added to each gel pieces and were incubated overnight at $37{ }^{\circ} \mathrm{C}$. The tryptic digest was extracted by using $90 \%$ ACN, $0.1 \%$ TFA for 60 min with vortex, twice and dried with speed Vac. The extracted peptides were then cleaned with ZipTip C18 (Millipore) with 4ul of acetonitrile-water (v/v, 0.1\% TFA) and diluted with $6 \mathrm{uL}$ of $0.1 \%$ formic acid.

\section{In-solution Protein Digestion}

For in-solution digestion, $400 \mu \mathrm{g}$ of mitochondrial protein was digested and divided into three equal parts for PLRP separations. Each aliquot was then digested with trypsin (Promega, Madison, WI, USA) that was dissolved in 50nM ABC buffer at the ratio of 1:50, for 18 hours at $37^{\circ} \mathrm{C}$. The digest was then first acidified with trifluoroacetic acid (TFA), then peptide desalting was done by solid-phase extraction using a homemade spin mini column that was filled with approx. 200mg of Vydac c18 packing material (Sigma Aldrich, St. Louis, MO, USA). The peptides were eluted with 400ul of 
acetonitrile-water, 70:30 (v/v, 0.1\% TFA) after washing three times with 500ul of water $(0.1 \%$ TFA $)$. The samples were stored at $-80{ }^{\circ} \mathrm{C}$ until further use.

\section{High-pH Reversed-Phase Chromatography (PLRP)}

The peptide solution eluted from in-solution protein digestion was reduced to approximately 10ul which was then mixed with 10ul of PLRP buffer A. Buffer A mobile phase is a mixture of water: ammonium hydroxide (30\%); (99.8:0.2, v:v), $\mathrm{pH} 10)$. The peptide and buffer mixture was then injected into the polymer-based reverse phase column (PLRP-S, 5 $\mu \mathrm{m}, 100 \mathrm{~A}, 150 \mathrm{x}$ 2.1mm) from Agilent Technologies (Santa Clara, CA, USA). Elution of peptide was done with a linear gradient of buffer B, which consists of acetonitrile: water: ammonium hydroxide (30\%); (80:19.8:0.2, v/v/v), $\mathrm{pH} 10$, from 0$90 \%$ for 95 minutes, which was followed by 5 min isocratic elution, 5 minutes reequilibration (90\%-0\% B) and $10 \mathrm{~min}$ isocratic. Peptides fractions, 15 fractions per samples, were collected using HPLC fraction collector (Spectra/Chrom, Model CF-1, Spectrum Chromatography, Houston, TX, USA). The peptide fractions were dried in a vacuum centrifuge, re-suspended in $10 \mathrm{ul}$ water with $0.1 \%$ TFA and stored at $-80{ }^{\circ} \mathrm{C}$ until ready for analysis.

\section{LC-MS/MS}

After the first dimensional separation of the peptides, the second dimensional separation was done by home-packed Magic C18 (5 $\mu \mathrm{m}, 200$ A, Michrom Bioresources, Auburn, CA, USA) capillary column (100 mm $\times 75 \mu \mathrm{m}$ I.D.) (New objective, Woburn, MA, USA). Four microliters of peptide digest were injected manually into nano injector (Idex, Oak Harbor, WA, USA). A flow rate of $300 \mathrm{~nL} /$ minute was used to elute the peptides with linear gradient for $95 \mathrm{~min}$ from solvent A ((water: acetic acid: formic acid), (97.9:2:0.1), v/v/v) to solvent B (water: acetic acid: formic acid), (9.9:90:0.1, v/v/v). LTQ ion trap mass spectrometer (Thermo Fisher Scientific, Waltham, MA, USA) was used for mass analysis in a data-dependent scan mode with MS scan of 400-200m/z. The 7 most intense ions in each MS spectrum were automatically selected for collision-induced dissociation.

\section{Bioinformatics}

The raw data from the mass spectrometer was analyzed by Proteome Discoverer software version 1.4 (Thermo Fischer Scientific, Waltham, MA, USA). Sequest HT for rat database (swiss-prot; number of protein sequences 28,885; version 06/27/2013) that was digested in-silico with trypsin was used. The criteria to select the protein in proteome discoverer are as follows; only fully tryptic peptide was considered, precursor error tolerance was selected to $1.5 \mathrm{Da}$, product ion tolerance was $0.8 \mathrm{Da}$, peptide modification selected for dynamic modifications were methionine oxidation, lysine acetylation and that static modification was carbamidomethylation of cysteine. Percolator was used to 
calculate the false discovery rate for matched peptides which considered peptides with PEP score of 0.01 or lower to be a positive match.

Data analysis was done by GraphPad Prism (v5.0.4; La Jolla, CA, USA). To calculate the GRAVY peptide and protein score the parameters described by Kyte and Doolittle were used which calculated the scores based upon hydrophobicity of amino acid. The PANTHER protein classification system was used to analyze the Gene Ontology (GO) annotations which identified the functional annotations. TargetP server was used to predict the sub-cellular location of the identified proteins. Cytoscape (v3.0.2) plug-in ClueGo was used for the biological interpretation of identified proteins. SwissProt and primary literature were used to obtain information on protein functions.

\section{Results and Discussion}

In this study, we evaluated three multidimensional bioanalytical platforms for protein profiling. The overall experimental scheme is presented in Figure 2-3. Both gelbased and gel-free prefractionation methods were included in our study. For the gel-based method, we used IEF and SDS-PAGE analysis that separated the intact proteins in the first dimension. After the proteins were separated, the gels were divided equally into 15 fractions and processed for in-gel digestion with trypsin. The tryptic peptide mixtures were separated in the second dimension with reverse phase LC interfaced with MS/MS. The data from mass spectrometric analysis were analyzed by proteome discoverer, and the list of identified proteins was generated for each gel section. The list of proteins identified from each section was finally combined to generate a final list of proteins for each bioanalytical platform. For the gel-free pre-fractionation, the protein mixture was first digested with trypsin and peptide level separation was performed by reverse phase $\mathrm{LC}$ at high $\mathrm{pH}$ (PLRP). In this analytical platform also 15 peptide fractions were collected which were then separated in the second dimension by reverse phase LC and analyzed by mass spectrometer. As the gel-based methods, the proteins lists identified in each of the 15 fractions were combined, and a list of proteins for this analytical platform was created. Three technical replicates for each platform were performed. We also analyzed unfractionated samples by mass spectrometric analysis and compared it to the fractionated samples. Fractionation helped in the identification of a higher number of proteins and gave better proteome coverage (Table 2-1). Also, various proteins related to important functional modules such as TIM/TOM protein import, and fusion/fission proteins could not be identified in unfractionated samples.

\section{Summary of Protein Identification Results}

As shown in Table 2-2, for each of the bioanalytical platform, 15 peptide mixtures were analyzed by LC-MS/MS in three technical replicates. The total number of LC-MS/MS analyses performed in this this bioanalytical comparison study was 135 .

Percolator, a new addition to proteome discoverer, was used to calculate the protein false 


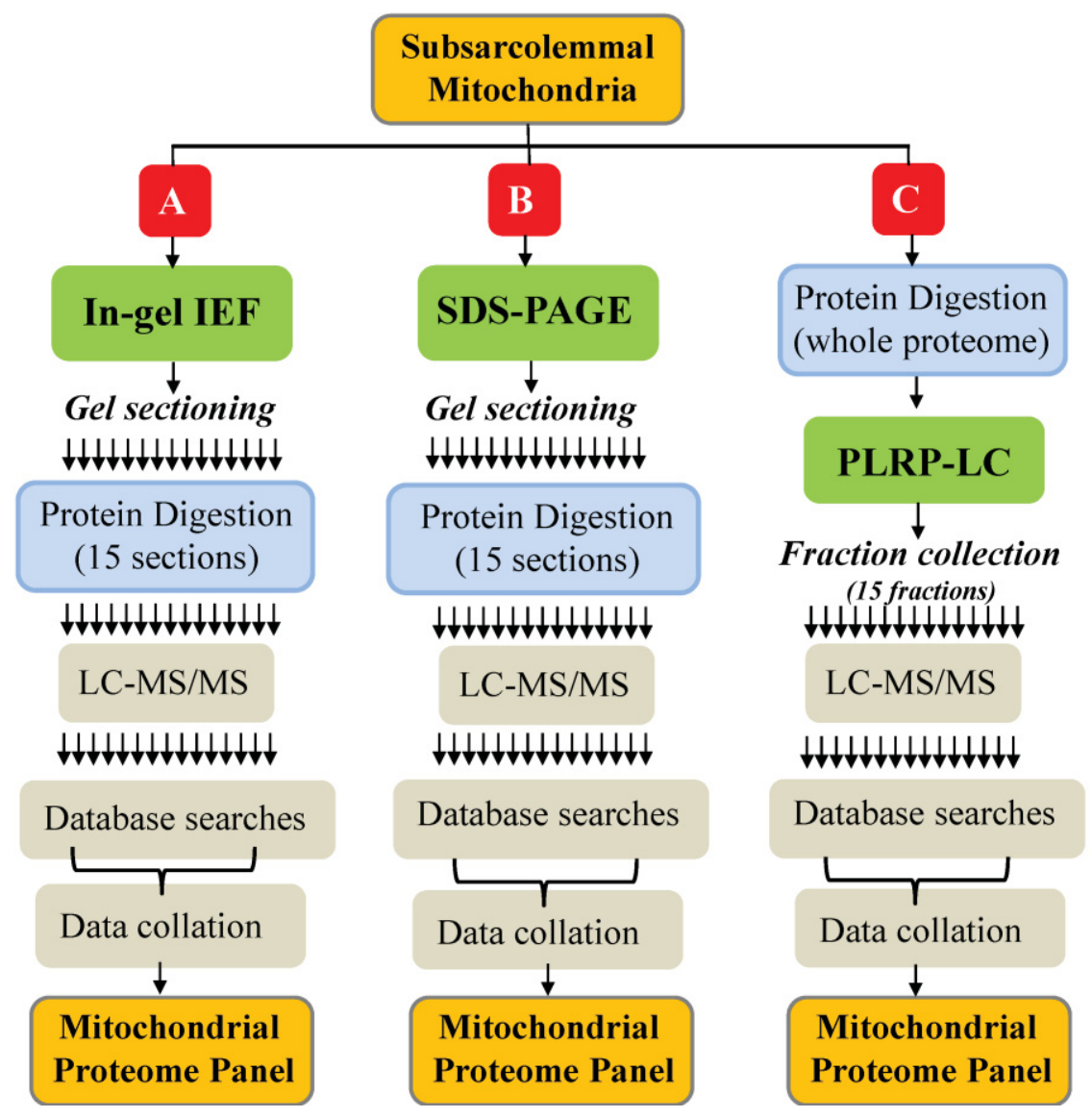

Figure 2-3. Experimental scheme for the comparative evaluation of three bioanalytical platforms for mitochondrial proteome profiling.

(A) or sodium dodecyl sulfate-polyacrylamide gel electrophoresis (SDS-PAGE) (B); or by PLRP (polymeric reversed-phase liquid chromatography at high $\mathrm{pH}$ ) at the peptide level following proteolytic digestion $(\mathbf{C})$. Fifteen gel sections $(\mathbf{A}, \mathbf{B})$ or 15 liquid fractions (C) were produced, and the peptide mixtures were analyzed by liquid chromatographymass spectrometry/mass spectrometry (LC-MS/MS). The LC-MS/MS data were used to identify the proteins sampled with each platform. Three technical replicates were performed with each platform. Multiple arrows indicate multiple fractions/sections. 
Table 2-1. Total proteins identified in fractionated vs. unfractionated samples.

\begin{tabular}{cc}
\hline Sample Processing & Number of Proteins Identified \\
\hline Unfractionated & 213 \\
Fractionation by SDS-PAGE & 890 \\
\hline
\end{tabular}

Table 2-2. Experimental design: numbers of samples for each platform.

\begin{tabular}{ccccc}
\hline & \multicolumn{3}{c}{ Technical Replicate } & \\
\cline { 2 - 4 } Platform & Repeat-1 & Repeat-2 & Repeat-3 & Total Run \\
\hline SDS-PAGE & 15 & 15 & 15 & 45 \\
PLRP & 15 & 15 & 15 & 45 \\
IEF & 15 & 15 & 15 & 45 \\
\hline
\end{tabular}


discovery rates (FDR). The peptides were deemed positive if the PEP scores were $<0.01$ for fully tryptic peptides. A total number of 1043 unique proteins were identified by all the platforms combined. A full list of proteins identified with each platform is provided in Supplementary Table Listing Number of Proteins Identified.

There was a pronounced difference in the performance of the three analytical platforms in mapping the total proteome. Table 2-3 summarizes the protein identification results. SDS-PAGE based analytical platform identified 890 proteins, IEF based analytical platform identified 600 proteins and PLRP identified 450 proteins in total. Therefore, SDS-PAGE based platform identified the highest number of proteins among the three platforms. In addition, the SDS-PAGE based platform also identified the highest number of peptides giving the highest protein coverage. The total peptide counts for IEF based platform was 50\% less than that of SDS-PAGE based platform. Also, there was only $32.7 \%$ overlap of proteome coverage between the three platforms. The distribution of unique proteins identified by each platform and the protein overlaps among all the three individual platforms are shown in Figure 2-4.

\section{Intrinsic Chemical Properties of Identified Proteins}

Three different intrinsic chemical properties of the proteins identified with each platform viz. GRAVY (grand average of hydropathy) Score; Molecular weight (Mw); and isoelectric point $(\mathrm{pI})$ were assessed. The identified proteins by these three platforms showed similar trends based on these intrinsic parameters (Figure 2-5). The only major difference seen among these platforms was in the high molecular weight section, the SDS-PAGE based platform identified a significantly greater number of high molecular weight proteins $(\mathrm{p}<0.001)$ (Figure 2-5B). Also, regardless of the bioanalytical platforms, a major number of proteins identified have $\mathrm{pI}$ values between 7 and 9 (Figure 2-5C).

\section{Protein Abundance}

Performance of the three bioanalytical platforms used in this study in terms of the dynamic range of protein coverage was assessed. For this purpose, the relative protein abundance was calculated using normalized spectral abundance factors (NSAF). Figure 2-6 shows that all the three platforms were able to sample proteins over a wide range of abundance level. In this scenario too the SDS-PAGE based platform was found to be superior as it was able to provide the most in-depth coverage of mitochondrial proteins with the dynamic range spanning five orders of magnitude, i.e., from $10^{-1}$ to $10^{-6}$.

\section{Categorization of the Identified Proteins}

The proteins in the panels identified by each bioanalytical platforms were analyzed for their cellular localizations by ClueGO [61] and, biological processes by 
Table 2-3. Summary of the performances of three gel-based and gel-free bioanalytical platforms for mitochondrial proteome mapping.

\begin{tabular}{lccc}
\hline \multicolumn{1}{c}{ Performance Characteristic } & SDS-PAGE & IEF & PLRP \\
\hline Number of proteins-----replicate 1 & 708 & 520 & 315 \\
Number of proteins-----replicate 2 & 726 & 468 & 330 \\
Number of proteins-----replicate 3 & 696 & 476 & 361 \\
Average \pm SD & $710 \pm 15$ & $488 \pm 28$ & $335 \pm 23$ \\
C.V. & $2.1 \%$ & $5.7 \%$ & $7.0 \%$ \\
Total number of proteins (all runs) & 890 & 600 & 450 \\
Total number of peptides (all runs) & 6023 & 3110 & 1671 \\
Proteome coverage overlap & $64 \%$ & $63 \%$ & $53 \%$ \\
Total number of proteins (all platform) & 1043 & - & - \\
Proteome coverage overlap (all platform) & $32.7 \%$ & & \\
\hline
\end{tabular}



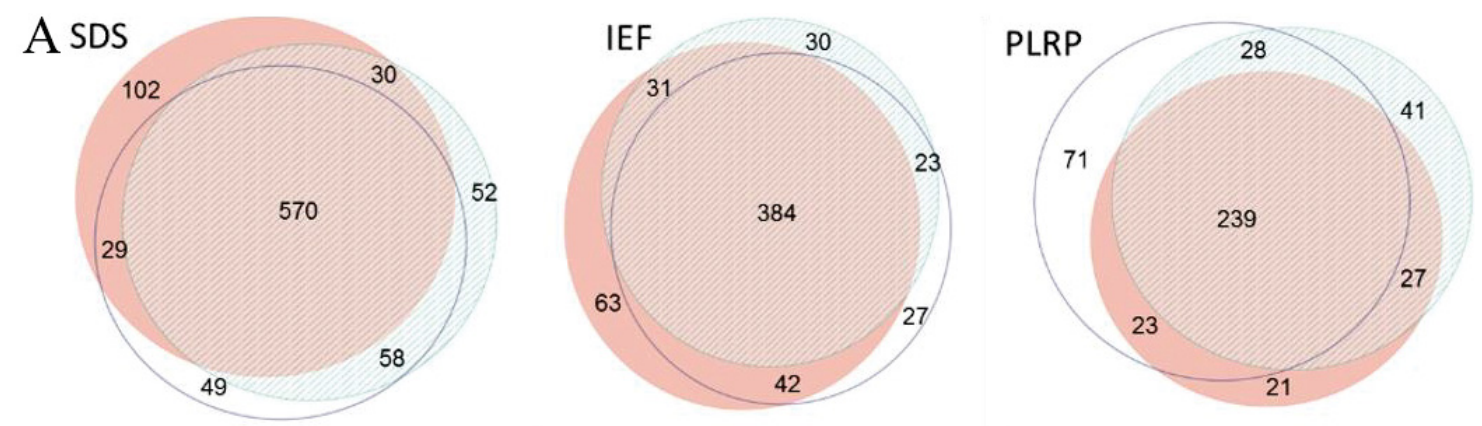

B

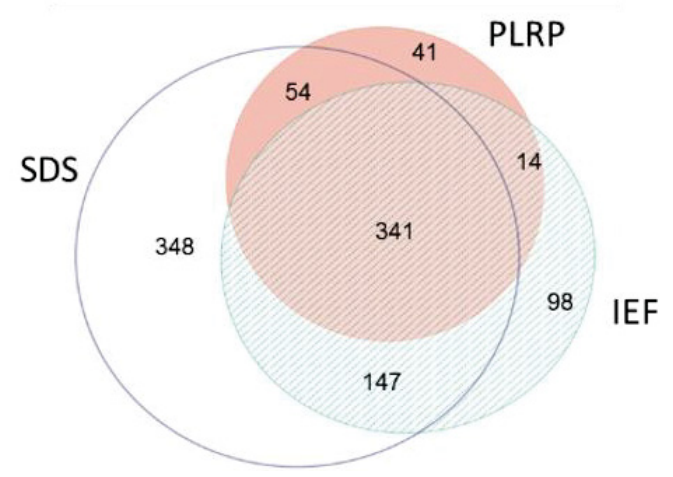

Figure 2-4. Venn diagrams showing the distribution of unique proteins identified by each platform.

(A) Top portion of the figure shows the overlap for each platform among the three technical replicates; (B) Bottom portion of the figure shows the overlap among the three individual platforms. 

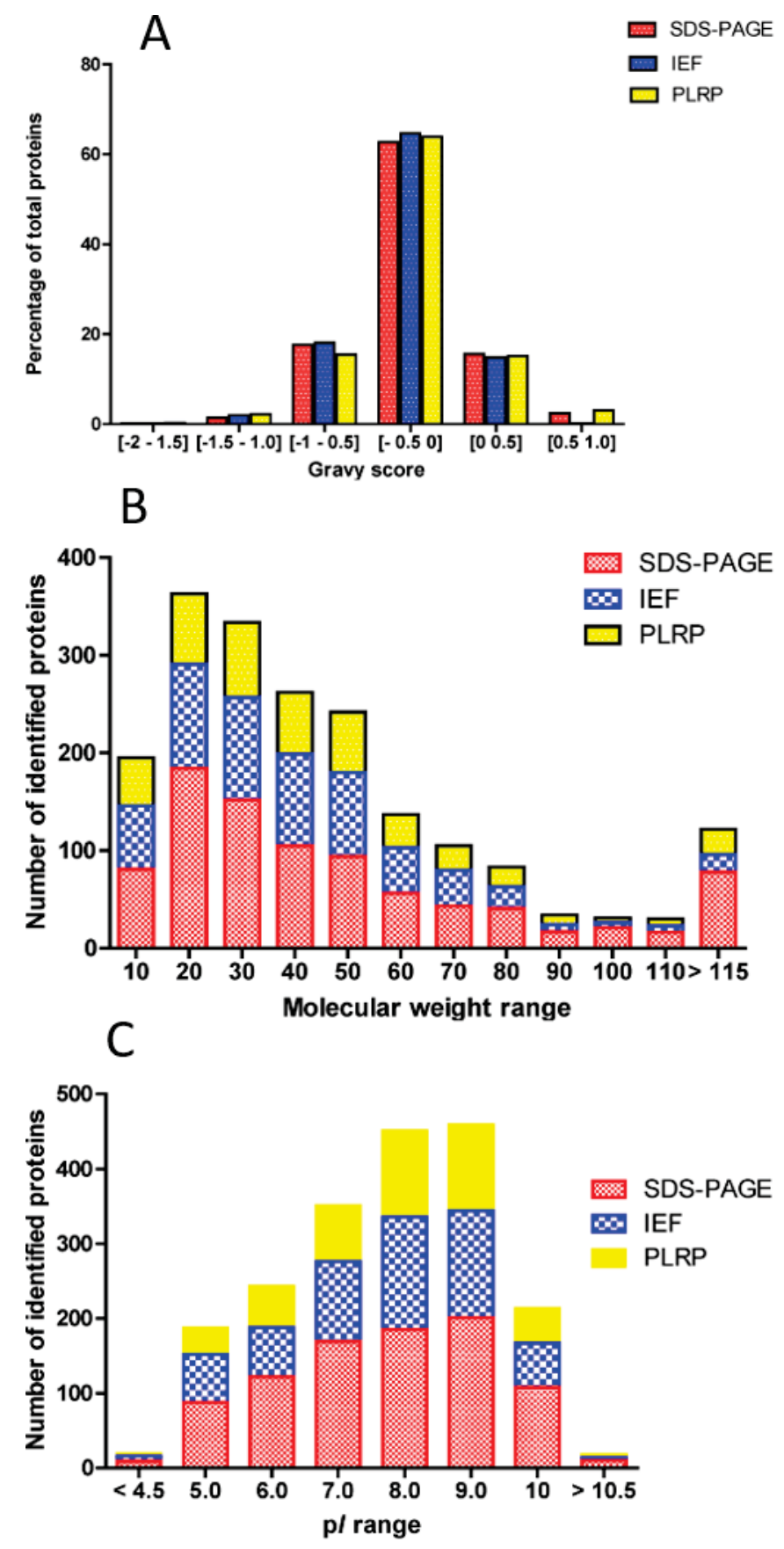

Figure 2-5. Distribution of intrinsic properties of the mitochondrial proteins identified by each platform.

(A) grand average of hydropathy (GRAVY) score; (B) MW (in kDa); (C) pI. 


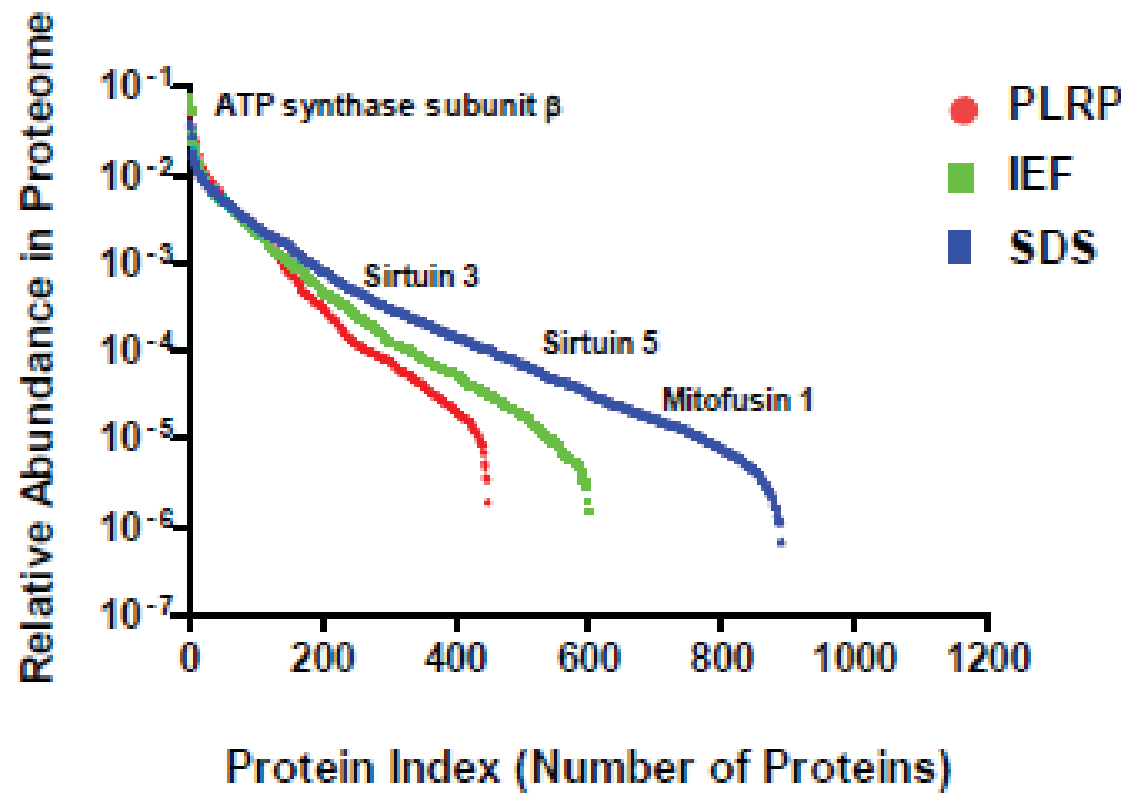

Figure 2-6. Relative abundance of mitochondrial proteins sampled with each platform. 
PANTHER [62]. The top cluster that was common to all the three bioanalytical platforms was metabolic processes (GO:008152). Around $41 \%$ to $49 \%$ of the total protein identified fall in this group, which is one of the main functions of mitochondria (Figure 2-7).

Similarly, the proteome panel obtained from our study was also analyzed for the presence of N-terminal mitochondrial targeting sequences (MTS) by using TargetP [63]. All the platforms showed similar results, i.e., among all the proteins identified by each platform around 50\% of the proteins identified contained MTS (Figure 2-8). MTS helps proteins migration towards mitochondria after their syntheses. However, there are other mechanisms that also transport proteins without $\mathrm{N}$-terminal MTS into the mitochondria [64].

\section{Selected Functional Characteristic of Mitochondrial Proteome Panel}

The catalog of protein panel from this study was from mitochondrial proteins. So, we selected five major functional modules of mitochondria and analyzed if the identified proteins belong to these modules. It was found that most of the identified mitochondrial proteins belonged to the electron transport chain (ETC)/oxidative phosphorylation (OXPHOS) module as proteins are one of the major clusters of the mitochondrial system. A total of 89 OXPHOS proteins were identified and are listed in Table 2-4. The oxidative phosphorylation metabolic pathway consists of five ETC/OXPHOS protein complexes, and the list of proteins, identified in our study included protein subunits from all five ETC/OXPHOS complexes. The proteins that belong to the component of mitochondrial ribosome were the second major functional modules identified by the proteome panel in which 39 members of the mitochondrial ribosomes were identified. Similarly, 17 members of the TIM/TOM protein machinery were identified in our study. These proteins play an important role in protein translocation into the mitochondria. The other important functional clusters are proteins that play a crucial role in mitochondrial fusion and fission. Dynamin-like $120 \mathrm{kDa}$ protein-OPA1, mitofusin-1 that belongs to mitochondria fission and fusion [65], along with other three proteins, were also identified. In addition, sirtuin 3 and sirtuin 5 that belong to a protein deacetylase family were identified in the study [66].

\section{Conclusion}

Over the past 15 years, mass spectrometry-based workflows (i.e., workflows that by-pass traditional 2D-PAGE) have been increasingly used for protein identification and quantification in proteomics. In order to effectively probe a complex proteome, upstream pre-fractionation may be considered. There are a number of different pre-fractionation strategies available. In our study, we compared three multidimensional bioanalytical platforms that incorporate different means of protein/peptide fractionation. We selected IEF, SDS-PAGE, and PLRP separations. All three methods were easy to set up in our laboratory because no specialized equipment was required to perform these separations, and all three methods are effective for separation of complex mixtures. The number of 


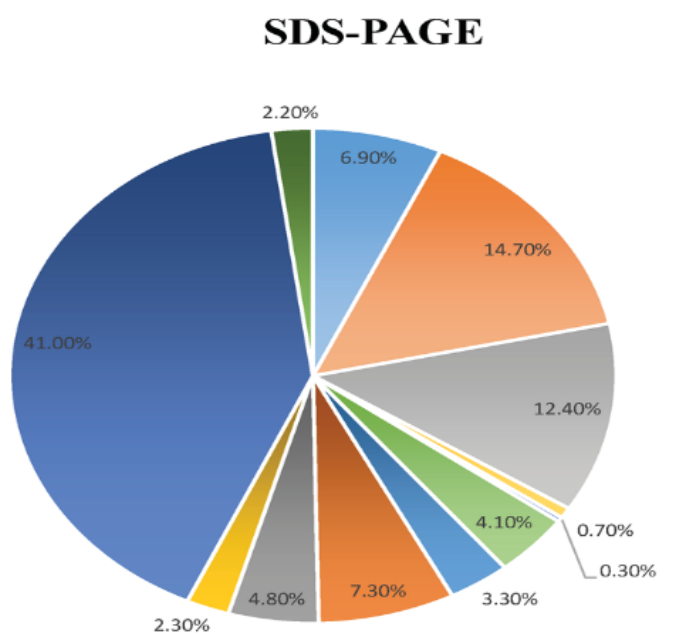

PLRP

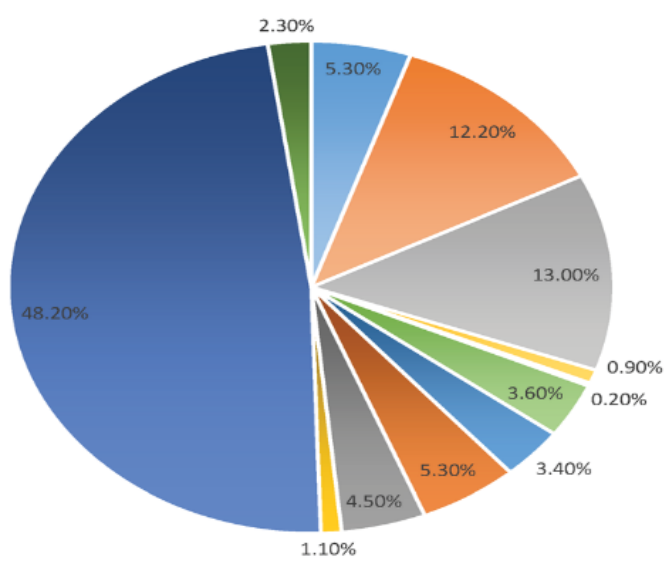

IEF

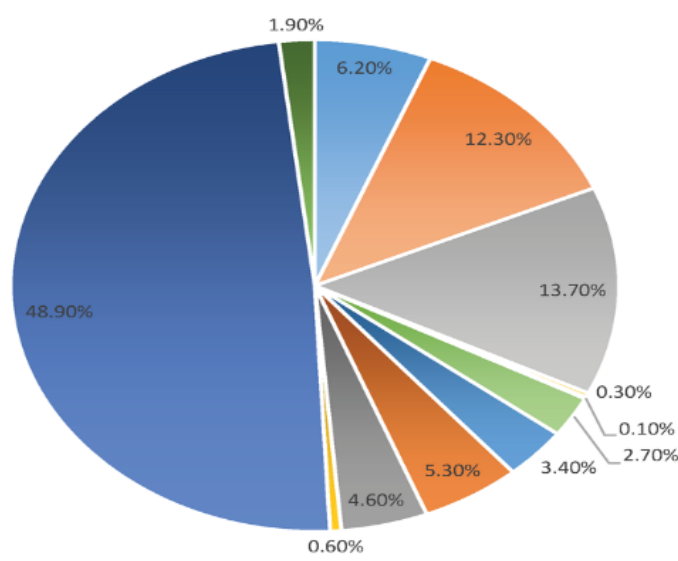

= cellular component organization or

biogenesis (GO:0071840)

= cellular process (GO:0009987)

- localization (GO:0051179)

" apoptotic process (GO:0006915)

- reproduction (GO:0000003)

- biological regulation (GO:0065007)

- response to stimulus (GO:0050896)

- developmental process (GO:0032502)

- multicellular organismal process

(GO:0032501)

- biological adhesion (GO:0022610)

- metabolic process (GO:0008152)

- immune system process (GO:0002376)

cellular component organization or biogenesis (GO:0071840)

- cellular process (GO:0009987)

- localization (GO:0051179)

" apoptotic process (GO:0006915)

- reproduction (GO:0000003)

" biological regulation (GO:0065007)

- response to stimulus (GO:0050896)

" developmental process (GO:0032502)

multicellular organismal process

(GO:0032501)

" biological adhesion (GO:0022610)

- metabolic process (GO:0008152

- immune system process (GO:0002376)

" cellular component organization or biogenesis (GO:0071840)

" cellular process (GO:0009987)

- localization (GO:0051179)

apoptotic process (GO:0006915)

reproduction (GO:0000003)

biological regulation (GO:0065007)

- response to stimulus (GO:0050896)

- developmental process (GO:0032502)

- multicellular organismal proces (GO:0032501)

" biological adhesion (GO:0022610)

- metabolic process (GO:0008152)

- immune system process (GO:0002376

Figure 2-7. Gene ontology categorization of the identified proteins according to the biological process. 


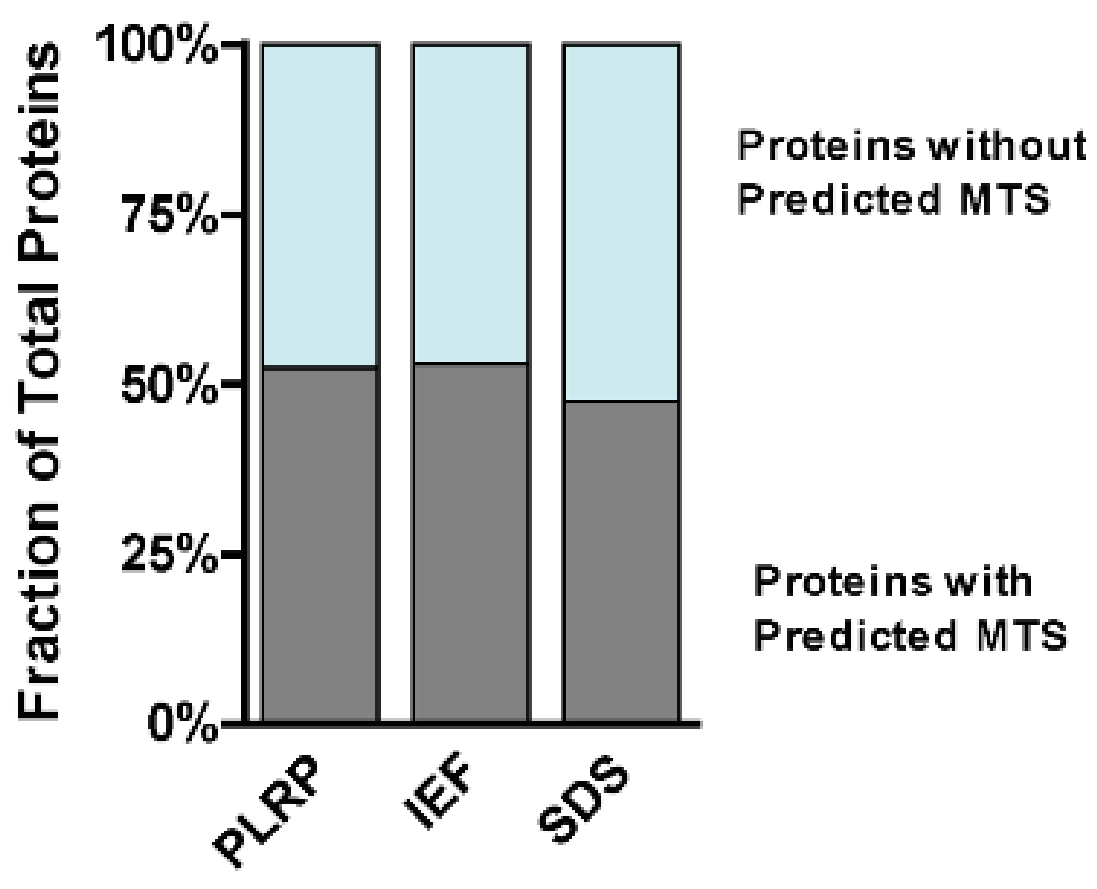

Figure 2-8. Distribution of proteins according to the presence of N-terminal mitochondrial targeting sequence (MTS). 
Table 2-4. Representative proteins from selected functional classes.

\begin{tabular}{|c|c|}
\hline Accession & Description \\
\hline P15999 & ATP synthase subunit alpha, mitochondrial \\
\hline G3V6D3 & ATP synthase subunit beta \\
\hline Q66HF1 & NADH-ubiquinone oxidoreductase 75 kDa subunit, mitochondrial \\
\hline Q920L2 & Succinate dehydrogenase [ubiquinone] flavoprotein subunit, mitochondrial \\
\hline P32551 & Cytochrome b-c1 complex subunit 2 , mitochondrial \\
\hline P19511 & ATP synthase subunit $b$, mitochondrial \\
\hline Q68FY0 & Cytochrome b-c1 complex subunit 1 , mitochondrial \\
\hline P35435 & ATP synthase subunit gamma, mitochondrial \\
\hline P20788 & Cytochrome b-c1 complex subunit Rieske, mitochondrial \\
\hline Q5BK63 & NADH dehydrogenase [ubiquinone] 1 alpha subcomplex subunit 9, mitochondrial \\
\hline Q06647 & ATP synthase subunit $\mathrm{O}$, mitochondrial \\
\hline Q5XIH3 & NADH dehydrogenase (Ubiquinone) flavoprotein 1 \\
\hline D3ZG43 & NADH dehydrogenase (Ubiquinone) Fe-S protein 3 (Predicted), isoform CRA c \\
\hline P31399 & ATP synthase subunit $d$, mitochondrial \\
\hline P00406 & Cytochrome c oxidase subunit 2 \\
\hline Q2TA68 & Dynamin-like $120 \mathrm{kDa}$ protein, mitochondrial \\
\hline Q561S0 & NADH dehydrogenase [ubiquinone] 1 alpha subcomplex subunit 10 , mitochondrial \\
\hline P10888 & Cytochrome $\mathrm{c}$ oxidase subunit 4 isoform 1 , mitochondrial \\
\hline P11240 & Cytochrome c oxidase subunit $5 \mathrm{~A}$, mitochondrial \\
\hline Q641Y2 & NADH dehydrogenase [ubiquinone] iron-sulfur protein 2 , mitochondrial \\
\hline D4A0T0 & Protein Ndufb 10 \\
\hline Q6PDU7 & ATP synthase subunit g, mitochondrial \\
\hline P21913 & Succinate dehydrogenase [ubiquinone] iron-sulfur subunit, mitochondrial \\
\hline D3ZE15 & Protein LOC100911483 \\
\hline D3ZLT1 & NADH dehydrogenase (Ubiquinone) 1 beta subcomplex, 7 (Predicted) \\
\hline Q63362 & NADH dehydrogenase [ubiquinone] 1 alpha subcomplex subunit 5 \\
\hline B0BNE6 & NADH dehydrogenase (Ubiquinone) $\mathrm{Fe}-\mathrm{S}$ protein 8 (Predicted), isoform CRA a \\
\hline Q5XIF3 & NADH dehydrogenase [ubiquinone] iron-sulfur protein 4 , mitochondrial \\
\hline F1LXA0 & NADH dehydrogenase (Ubiquinone) 1 alpha subcomplex, 12 (Predicted), isoform CRA b \\
\hline Q5RJN0 & NADH dehydrogenase (Ubiquinone) $\mathrm{Fe}-\mathrm{S}$ protein 7 \\
\hline P19234 & NADH dehydrogenase [ubiquinone] flavoprotein 2 , mitochondrial \\
\hline Q8SEZ0 & NADH-ubiquinone oxidoreductase chain 5 \\
\hline D3ZD09 & Cytochrome c oxidase subunit $6 \mathrm{~B} 1$ \\
\hline P12075 & Cytochrome c oxidase subunit $5 \mathrm{~B}$, mitochondrial \\
\hline Q80W89 & NADH dehydrogenase [ubiquinone] 1 alpha subcomplex subunit 11 \\
\hline D4A565 & NADH dehydrogenase (Ubiquinone) 1 beta subcomplex, 5 (Predicted), isoform CRA_b \\
\hline D4A7L4 & NADH dehydrogenase (Ubiquinone) 1 beta subcomplex, 11 (Predicted) \\
\hline B2RYS2 & Cytochrome b-c1 complex subunit 7 \\
\hline B2RYS8 & NADH dehydrogenase (Ubiquinone) 1 beta subcomplex 8 \\
\hline P29419 & ATP synthase subunit e, mitochondrial \\
\hline B5DEL8 & NADH dehydrogenase (Ubiquinone) Fe-S protein 5 \\
\hline B2RYW3 & NADH dehydrogenase (Ubiquinone) 1 beta subcomplex, 9 \\
\hline B2RZD6 & Ndufa4 protein \\
\hline H9KVF8 & NADH-ubiquinone oxidoreductase chain 1 (Fragment) \\
\hline Q5PQZ9 & $\mathrm{NADH}$ dehydrogenase [ubiquinone] 1 subunit $\mathrm{C} 2$ \\
\hline Q7TP78 & Aa2-258 \\
\hline P05508 & NADH-ubiquinone oxidoreductase chain 4 \\
\hline G3V7Y3 & ATP synthase subunit delta, mitochondrial \\
\hline P10817 & Cytochrome c oxidase subunit $6 \mathrm{~A} 2$, mitochondrial (Fragment) \\
\hline P11951 & Cytochrome c oxidase subunit $6 \mathrm{C}-2$ \\
\hline D3ZAF6 & ATP synthase subunit $f$, mitochondrial \\
\hline D4A3V2 & NADH dehydrogenase [ubiquinone] 1 alpha subcomplex subunit 6 \\
\hline D3ZZ21 & NADH dehydrogenase (Ubiquinone) 1 beta subcomplex, 6 (Predicted) \\
\hline Q5M9I5 & Cytochrome b-c1 complex subunit 6 , mitochondrial \\
\hline P35171 & Cytochrome $\mathrm{c}$ oxidase subunit $7 \mathrm{~A} 2$, mitochondrial \\
\hline A9UMV9 & Ndufa7 protein \\
\hline Q7TQ16 & Cytochrome b-c1 complex subunit 8 \\
\hline
\end{tabular}




\section{Table 2-4 (Continued.)}

\begin{tabular}{|c|c|}
\hline Accession & Description \\
\hline D3ZS58 & NADH dehydrogenase [ubiquinone] 1 alpha subcomplex subunit 2 \\
\hline P11608 & ATP synthase protein 8 \\
\hline H9KVF5 & NADH-ubiquinone oxidoreductase chain 3 (Fragment) \\
\hline G3V640 & Mitochondrial import inner membrane translocase subunit TIM44 \\
\hline Q5U2X7 & Mitochondrial import inner membrane translocase subunit Tim21 \\
\hline D3ZJX5 & Protein Timm50 \\
\hline P21571 & ATP synthase-coupling factor 6, mitochondrial \\
\hline D3ZYX8 & Cytochrome c oxidase subunit VIIa polypeptide 2 like (Predicted), isoform CRA_e \\
\hline Q75Q41 & Mitochondrial import receptor subunit TOM22 homolog \\
\hline P05503 & Cytochrome c oxidase subunit 1 \\
\hline Q6PCU8 & NADH dehydrogenase [ubiquinone] flavoprotein 3, mitochondrial \\
\hline P11662 & NADH-ubiquinone oxidoreductase chain 2 \\
\hline P80432 & Cytochrome c oxidase subunit $7 \mathrm{C}$, mitochondrial \\
\hline P05505 & Cytochrome $\mathrm{c}$ oxidase subunit 3 \\
\hline O08776-4 & Isoform 4 of NADH dehydrogenase [ubiquinone] 1 alpha subcomplex assembly factor 3 \\
\hline P52504 & NADH dehydrogenase [ubiquinone] iron-sulfur protein 6 , mitochondrial \\
\hline D3ZN43 & NADH dehydrogenase (ubiquinone) complex I, assembly factor 6 \\
\hline B2RZ31 & Protein Sirt3 \\
\hline P84817-3 & Isoform 3 of Mitochondrial fission 1 protein \\
\hline P62076 & Mitochondrial import inner membrane translocase subunit Tim13 \\
\hline Q9WV97 & Mitochondrial import inner membrane translocase subunit Tim9 \\
\hline Q62760 & Mitochondrial import receptor subunit TOM20 homolog \\
\hline F1M8L6 & Protein Ndufa3 \\
\hline O35092 & Mitochondrial import inner membrane translocase subunit Tim17-A \\
\hline G3V9S0 & Cytochrome b5 reductase 1 \\
\hline Q06645 & ATP synthase lipid-binding protein, mitochondrial \\
\hline Q9NQR8 & NADH dehydrogenase [ubiquinone] 1 alpha subcomplex assembly factor 4 \\
\hline R9PXR4 & Mitochondrial import receptor subunit TOM70 \\
\hline G3V644 & NADH dehydrogenase (Ubiquinone) flavoprotein 3-like, isoform CRA_a \\
\hline Q6PCT8 & Succinate dehydrogenase [ubiquinone] cytochrome b small subunit, mitochondrial \\
\hline D3ZTW7 & ATP synthase mitochondrial F1 complex assembly factor 2 (Predicted), isoform CRA_c \\
\hline O35093 & Mitochondrial import inner membrane translocase subunit Tim23 \\
\hline F1LWG4 & $\begin{array}{l}\text { NADH dehydrogenase (Ubiquinone) } 1 \text { alpha subcomplex, assembly factor } 1 \text { (Predicted), isoform } \\
\text { CRA_a }\end{array}$ \\
\hline P62078 & Mitochondrial import inner membrane translocase subunit Tim8 B \\
\hline Q9WVA1 & Mitochondrial import inner membrane translocase subunit Tim8 A \\
\hline Q68FX9 & NAD-dependent protein deacylase sirtuin-5, mitochondrial \\
\hline Q8R4Z9 & Mitofusin-1 \\
\hline Q5XIM4 & ATP synthase subunit $\mathrm{s}$, mitochondrial \\
\hline A9UMV7 & Protein Uqcr11 \\
\hline B2RYU0 & NADH dehydrogenase (Ubiquinone) 1 beta subcomplex, 2 (Predicted), isoform CRA_b \\
\hline D3ZZV1 & Mitochondrial import inner membrane translocase subunit TIM16 \\
\hline Q7H113 & NADH-ubiquinone oxidoreductase chain 4 \\
\hline P29418 & ATP synthase subunit epsilon, mitochondrial \\
\hline D3ZY50 & ATP synthase mitochondrial F1 complex assembly factor 1 (Predicted), isoform CRA_a \\
\hline P05504 & ATP synthase subunit a \\
\hline Q5I0I4 & ATP synthase subunit s-like protein \\
\hline Q5RJQ7 & Succinate dehydrogenase assembly factor 2 , mitochondrial \\
\hline G3V8F5 & Mitochondrial import receptor subunit TOM40 homolog \\
\hline P10818 & Cytochrome c oxidase subunit $6 \mathrm{~A} 1$, mitochondrial \\
\hline G3V9S6 & Protein Ndufa 1 \\
\hline Q5BJN5 & Mitochondrial intermembrane space import and assembly protein 40 \\
\hline Q9JKW1 & Mitochondrial import inner membrane translocase subunit Tim22 \\
\hline A4F267 & Mitochondrial import receptor subunit TOM40B \\
\hline Q5XI79 & NADH dehydrogenase [ubiquinone] complex I, assembly factor 7 \\
\hline Q4KM98 & Mitochondrial fission factor \\
\hline Q5XII9 & Mitochondrial fission regulator 1-like \\
\hline B2RYU0 & NADH dehydrogenase (Ubiquinone) 1 beta subcomplex, 2 (Predicted), isoform CRA_b \\
\hline
\end{tabular}


Table 2-4. (Continued.)

\begin{tabular}{|c|c|}
\hline Accession & $\begin{array}{c}\text { Description } \\
\end{array}$ \\
\hline B2RYU0 & NADH dehydrogenase (Ubiquinone) 1 beta subcomplex, 2 (Predicted), isoform CRA_b \\
\hline D3ZZV1 & Mitochondrial import inner membrane translocase subunit TIM16 \\
\hline Q7H113 & NADH-ubiquinone oxidoreductase chain 4 \\
\hline P29418 & ATP synthase subunit epsilon, mitochondrial \\
\hline D3ZY50 & ATP synthase mitochondrial F1 complex assembly factor 1 (Predicted), isoform CRA_a \\
\hline P05504 & ATP synthase subunit a \\
\hline Q5I0I4 & ATP synthase subunit s-like protein \\
\hline Q5RJQ7 & Succinate dehydrogenase assembly factor 2 , mitochondrial \\
\hline G3V8F5 & Mitochondrial import receptor subunit TOM40 homolog \\
\hline P10818 & Cytochrome c oxidase subunit $6 \mathrm{~A} 1$, mitochondrial \\
\hline G3V9S6 & Protein Ndufal \\
\hline Q5BJN5 & Mitochondrial intermembrane space import and assembly protein 40 \\
\hline Q9JKW1 & Mitochondrial import inner membrane translocase subunit Tim22 \\
\hline A4F267 & Mitochondrial import receptor subunit TOM40B \\
\hline Q5XI79 & NADH dehydrogenase [ubiquinone] complex I, assembly factor 7 \\
\hline Q4KM98 & Mitochondrial fission factor \\
\hline Q5XII9 & Mitochondrial fission regulator 1-like \\
\hline B2GV62 & Mitochondrial ribosomal protein L20 \\
\hline Q63750 & 39 S ribosomal protein $\mathrm{L} 23$, mitochondrial \\
\hline D3ZX69 & 39 S ribosomal protein $\mathrm{L} 10$, mitochondrial \\
\hline $\mathrm{P} 0 \mathrm{C} 2 \mathrm{C} 0$ & 39 S ribosomal protein L22, mitochondrial \\
\hline D4ABM5 & Mitochondrial ribosomal protein S34 (Predicted), isoform CRA_a \\
\hline F1LMZ4 & Ribosome-releasing factor 2 , mitochondrial \\
\hline Q9EPJ3 & $28 \mathrm{~S}$ ribosomal protein $\mathrm{S} 26$, mitochondrial \\
\hline Q6PDW6 & $39 \mathrm{~S}$ ribosomal protein $\mathrm{L} 17$, mitochondrial \\
\hline D4A131 & Mitochondrial ribosomal protein L4 (Predicted), isoform CRA_b \\
\hline Q7TQ82 & 28S ribosomal protein $\mathrm{S} 10$, mitochondrial \\
\hline B2RZ57 & Mitochondrial ribosomal protein L18 \\
\hline Q5I0K8 & $28 \mathrm{~S}$ ribosomal protein $\mathrm{S} 7$, mitochondrial \\
\hline D3ZMR9 & Mitochondrial ribosomal protein L21 (Predicted), isoform CRA_a \\
\hline D3ZYT2 & Mitochondrial ribosomal protein S5 (Predicted) \\
\hline D4A4B1 & Mitochondrial ribosomal protein L15 (Predicted), isoform CRA_c \\
\hline Q4G067 & Mitochondrial ribosomal protein L44 \\
\hline Q6AXT0 & 39S ribosomal protein $\mathrm{L} 37$, mitochondrial \\
\hline B0BN68 & Mitochondrial ribosomal protein S9 \\
\hline Q0ZFS4 & Mitochondrial ribosomal protein S33 (Predicted), isoform CRA_a \\
\hline Q5XFW4 & Mitochondrial ribosomal protein L13 \\
\hline Q5XIE3 & 39S ribosomal protein L11, mitochondrial \\
\hline D3ZIN7 & Mitochondrial ribosomal protein S23 (Predicted), isoform CRA_b \\
\hline Q5RK00 & 39S ribosomal protein L46, mitochondrial \\
\hline F6Q5K7 & Mitochondrial ribosomal protein S18B, isoform CRA_a \\
\hline P18665 & 39S ribosomal protein $\mathrm{L} 3$, mitochondrial \\
\hline Q5M818 & 39 S ribosomal protein L16, mitochondrial \\
\hline B0BN56 & $28 \mathrm{~S}$ ribosomal protein $\mathrm{S} 31$, mitochondrial \\
\hline Q5PQN9 & 39S ribosomal protein L38, mitochondrial \\
\hline B2RYT0 & Mitochondrial ribosomal protein S21 (Predicted), isoform CRA_a \\
\hline B2RYW4 & Mitochondrial ribosomal protein L53 \\
\hline $\mathrm{P} 0 \mathrm{C} 2 \mathrm{C} 1$ & 39S ribosomal protein L30, mitochondrial \\
\hline D3ZYL4 & Mitochondrial ribosomal protein L50 (Predicted) \\
\hline D4A3E8 & Mitochondrial ribosomal protein S27 (Predicted), isoform CRA_b \\
\hline D4A4V1 & Mitochondrial ribosomal protein S18C (Predicted), isoform CRA_a \\
\hline Q641X9 & 39S ribosomal protein L9, mitochondrial \\
\hline D3ZYT2 & Mitochondrial ribosomal protein S5 (Predicted) \\
\hline D4A4B1 & Mitochondrial ribosomal protein L15 (Predicted), isoform CRA_c \\
\hline
\end{tabular}

Note: Color codes: ETC/OXPHOS - ORANGE; FUSION/FISSION - GREEN; TIM/TOM PROTEIN IMPORT - BLUE; MITOCHONDRIAL RISOBOME - PURPLE; OTHERS-BLACK 
fractions generated in each platform was chosen to be 15 , which we considered to be a good balance between the time required to complete analyses of the multiple fractions generated from each proteome sample vs the enhancement of proteome coverage afforded by incorporation of these multidimensional separations. As expected, compared to proteome coverage attained with no up-stream fractionation, inclusion of multidimensional separation greatly increased the number of proteins being sampled. Our results demonstrate that SDS-PAGE based platform can provide the best proteome coverage when compared to the other platforms. The better performance of SDS-PAGE may be due to better protein solubility and protein denaturation due to the use of SDS. The IEF based platform also uses chaotropic agents that denatures the proteins. However, due to the strong denaturing property of SDS and smaller pore size of SDS-PAGE as compared to IEF gel strips, the former outperformed IEF based platform. The higher pore size of IEF increases the chance of loss of the protein from gel plug during sample processing which involves washing steps. However, when the gel-free method PLRP is compared with gel-based methods, both the gel-based methods outperformed the gel-free method. The better performance of gel-based methods attributes to the protein denaturation by chaotropic agents in the gel-based method and, more efficient sample proteolysis and incomplete samples recovery from PLRP column in the gel-free method.

Considerations of throughput are important when evaluating the suitability of a chosen platform for comparative proteome profiling, in which the analytical objectives include global-scale protein identification as well as relative quantification of protein expression levels in different samples, e.g. diseased vs. control specimens, cells treated with an exogenous agent postulated to induce proteome alterations, etc. Comparative proteomics studies necessitate examination of multiple replicates per sample group to obtain statistically significant data, and hence a large number of samples need to be analyzed by the chosen workflow. With the SDS-PAGE based platform set up as described in this Chapter, 15 LC-MS/MS analyses per each sample were performed to probe close to 900 proteins in this high proteome-complexity sample. It would be possible to apply this platform to comparative proteome profiling; however, from the analytical standpoint such study would represent a major time- and resource-demanding undertaking. A possible compromise between throughput vs. proteome coverage demands is to decrease the number of fractions (SDS-PAGE gel sections) processed in the workflow. Several studies have shown that using short SDS-PAGE, i.e., 5-10 minutes or $1 \mathrm{~cm}$ of separation instead of full separation $(15 \mathrm{~cm})$, in which 4 gel sections are procured, provides a reasonable proteome coverage and requires fewer LC-MS/MS analyses [67-69]. However, since the completion in 2014 of the study presented in this chapter, our laboratory acquired a new state-of-the-art LC-MS/MS system (Waters Acquity M-class UPLC coupled to Synapt G2 Si) that enabled a major transformation in the down-stream portion of our proteomics workflows. This LC-MS/MS instrument combines superior LC separation, additional separation dimension via ion mobility, high resolution/high mass accuracy mass spectrometry and DIA capabilities. Coverage of a complex proteome (i.e., the number of proteins identified and quantified) achievable with this LC-MS/MS system without additional up-stream fractionation is 3,000-3,500 proteins. For this reason, in the later portion of my research focused on qualitative and quantitative proteomics of ARPE-19 cells (Chapter 5), I applied the workflow that uses 
no fractionation but incorporates the Waters UPLC-Synapt G2-Si instrumentation.[70, $71]$. 


\section{CHAPTER 3. SCAVENGER RECEPTOR-MEDIATED UPTAKE AND EFFECTS OF OXLDL IN RETINAL PIGMENT EPITHELIAL CELLS}

\section{Introduction}

The human eye is an important and delicate sensory organ [72]. When light rays pass through the cornea, the lens helps to focus the light rays on the retina. The light is then converted into electrochemical impulses by the cells in the retina. The impulses are transferred to the brain via optic nerve which helps us to view any object [73]. Retina is the light sensitive tissue lining the back of the eye. In the center of the retina lies the macula. It is characterized by a central thinner dip, the fovea, which is the only area where sharp vision is possible (Figure 3-1A). It is the part of the eye that is responsible for central sharp visual acuity. Any process that damages the integrity of the macula, particularly the fovea, compromises central vision and visual acuity. Retina consists of several layers as follows: i) Inner limiting membrane, ii) Nerve fiber, iii) Ganglion cell layer, iv) Inner plexiform layer, v) Inner nuclear layer, vi) Outer plexiform layer, vii) Outer nuclear layer, viii) External limiting membrane, ix) Layer of rods and cones and $\mathrm{x}$ ) Retinal pigment epithelium [74]. Among these layers, only photoreceptor cells rods and cons are directly sensitive to light [75]. These photoreceptor cells are supported by the retinal pigment epithelium (RPE). The RPE is a hexagonal, pigmented monolayer which is tightly packed with tight junction and adherens junctions proteins and forms the outer blood-retinal barrier (BRB). RPE layer of the eye is the part of the human eye that is most affected due to Age-related Macular Degeneration (AMD). The RPE has an intimate connection with the photoreceptors on its apical side for numerous reasons. First, the RPE phagocytoses $10 \%$ of the photoreceptor outer segments every day to recycle the retinal chromophore and remove damages caused by photo-oxidation of lipids and proteins to ensure proper photoreceptor function. Second, the RPE supplies oxygen and metabolites (including the retinal chromophore) to the rod and cone photoreceptors and forms the blood-retinal barrier that isolates the retina from the systemic circulation [76]. The basolateral side of RPE lie the Bruch's membrane and choroid capillaries which form an important part of blood retinal barrier (BRB). It plays an important role in the uptake of ions and nutrients, removing the metabolic waste from subretinal space. It also produces immunosuppressive factors that preserve the immune privilege of the eye [77].

AMD is the leading cause of visual impairment in elderly populations. The progression of AMD results in irreversible damage to the macula of the eye [78]. AMD is the third largest cause of visual impairment worldwide. In the United States alone, AMD affects roughly 10 million individuals and over 2 million of these suffer from late-stage AMD which severely affects the quality of life and increases the public health burden. There is no effective treatment available for most AMD cases, and for those able to be treated, the treatment is far from $100 \%$ effective [79].

The early stages of AMD are termed age-related maculopathy or ARM. At the ARM stage, visual symptoms can be very limited, if present at all, even though RPE 

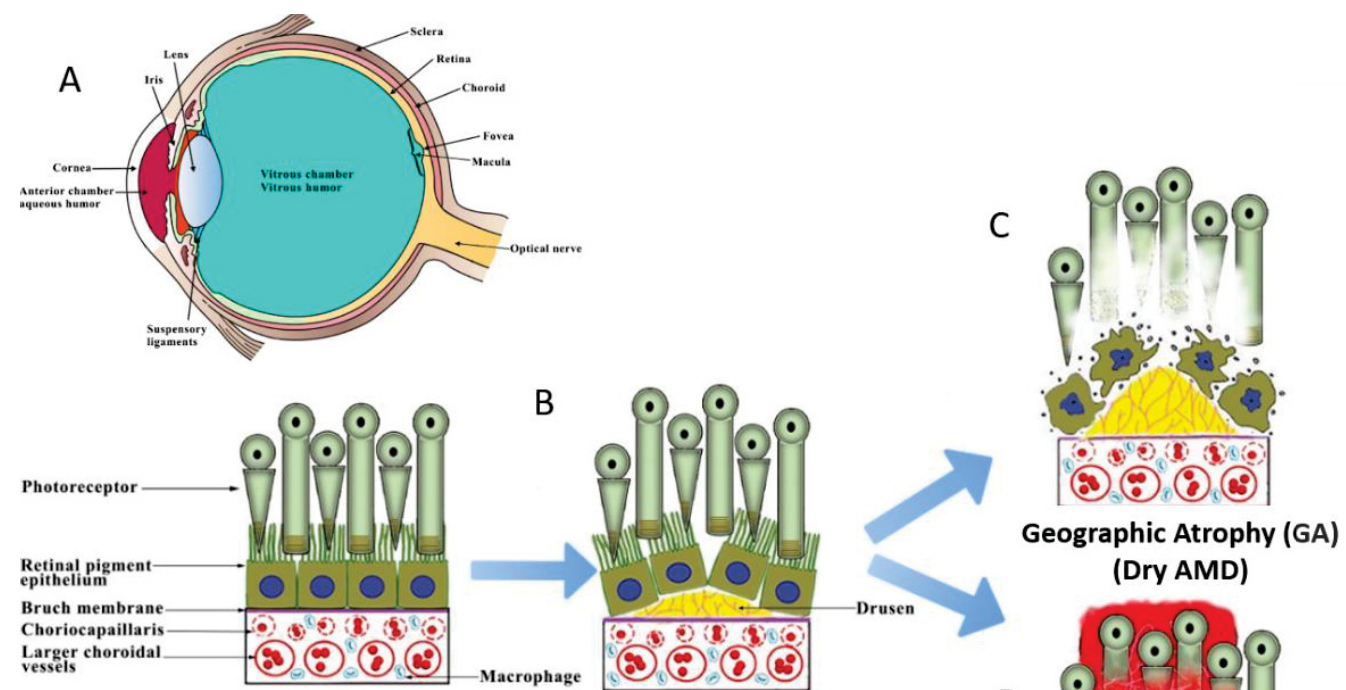

Normal Retina

Age Related Maculopathy

D

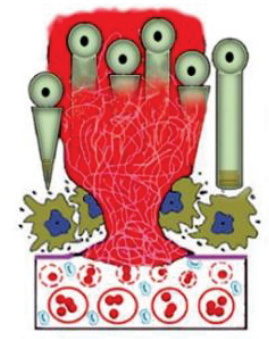

Choroidal Neovascularization (CNV)

(Wet AMD)

\section{Figure 3-1. Stages of AMD.}

(A) Diagram of the eye, shows the macula which is the central part of retina affected in AMD (B) Early drusen, the early stage which is called age related maculopathy.(C) Geographic Atrophy also called dry AMD which is characterized by the presence of drusen, (D) Choroidal Neovascularization also called wet AMD which is characterized by presence of blood vessel in the retina.

Image A reprinted with permission from an artwork by Holly Fischer obtained from https://commons.wikimedia.org/wiki/File:Three_Internal_chambers_of_the_Eye.png. License - creative commons (https://creativecommons.org/licenses/by/3.0/deed.en) $05 / 30 / 2018$. 
changes and deposits known as drusen (discussed further below) are already present at this stage (Figure 3-1B). At this stage, every form of the ARM is considered dry. As the tissue suffering increases and progresses in extent and severity over time, the advanced stages of AMD develop, leading to actual vision loss and other symptoms experienced by the affected patients. Advanced AMD is mainly classified into two broad forms: geographic atrophy and wet AMD. Geographic atrophy is the advanced stage of dry $\mathrm{AMD}$, characterized by progressive RPE atrophy and loss, which ultimately leads to functional compromise, the death of the overlying photoreceptors and vision loss. Extensive progression of the processes is sketched in Figure 3-1B. Drusen are often associated with geographic atrophy, and usually, their presence precedes RPE and photoreceptor loss. Geographic atrophy accounts for 80 to $90 \%$ of the advanced AMD cases [80]. Wet AMD, referred to also as neovascular (nv)AMD, accounts for the remaining $10-20 \%$ of advanced AMD and is characterized by the development of choroidal neovascularization (CNV), i.e., the growth of abnormal blood vessels from the choroid that penetrates the BM RPE and the macula. The newly formed blood vessels leak blood into the retina causing distortion of vision and eventually central vision loss (Figure 3-1C). Drugs that lead to CNV regression after it develops have been discovered and have become a mainstay of nvAMD treatment. However, ways to prevent the development of CNV as well as dry AMD have yet to be identified.

\section{Pathophysiology of AMD: Genetic and Environmental Factors}

AMD is a multifactorial disease that involves contributions from both environmental and genetic factors. Age, gender, race, cigarette smoking, diet, and various other cardiovascular risk factors have been identified as potential agents in the progression of AMD by various studies [79, 81]. The environmental exposure comprises around $30-55 \%$ of the attributable risk for the AMD whereas genetic factor is described to attribute the remaining $45-70 \%$ [82]. The major environmental factors that pose a risk for the development of AMD include increasing age, smoking, and low intake of dietary antioxidants. Other risks include higher body mass index, serum cholesterol, cataract surgery, cardiovascular diseases, hypertension, nutrition, and ultraviolet light exposure.

Age remains the major risk factor for AMD while cigarette smoking is the most strongly correlated environmental factor for AMD. It has been shown that disease progression is strongly influenced by smoking and the risk for dry AMD is reduced by smoking cessation [83]. Omega-3 fatty acid (FA) intake was found to be associated with lower risk of AMD in a recent epidemiologic study, showing correlations between AMD and lipid intake [84].

Occurrence of AMD also differs among race populations with the frequency of early signs of AMD at $4.2 \%$ in Hispanic populations, $5.4 \%$ in Caucasian populations and $2.4 \%$ in African American populations [81]. Thus, the different ethnic groups have different susceptibilities to AMD. It had been known for decades that genetic factors played a role in AMD. For instance, twin and family-based studies revealed a much greater risk of developing AMD among twins and first-degree relatives [85]. However, it 
was not until 2005 that the first direct link to a genetic factor was identified by Dr. Hageman's group . The group identified that mutations in the complement factor $\mathrm{H}$ $(\mathrm{CFH})$ gene caused a form of glomerulonephritis also characterized by the presence of extensive drusen is risk factor associated with AMD [86]. Following this discovery, genetic association analyses of AMD identified a non-synonymous single nucleotide polymorphism (SNP) at codon $402(\mathrm{Y} 402 \mathrm{H})$ in $\mathrm{CFH}$ as a major genetic risk factor in the AMD $[87,88]$. This new discovery paved the way towards a formal role for inflammation and immune response dysregulation in AMD pathogenesis. Following this initial discovery, 20 chromosomal regions have been identified that contain genetic variants associated with AMD. These 20 loci can be divided into five main subgroups as listed in Table 3-1.

\section{Oxidative Stress and AMD Pathology}

The factors that contribute to the cause of AMD such as smoking, diet, sunlight exposure, and vitamin D levels all are known to exhort oxidative stress. The Age-Related Eye Disease Study (AREDS) was one of the first studies that highlighted the role of oxidative stress as a disease contributor to macular degeneration. The study conducted by the National Eye Institute (NEI) showed that the use of antioxidant agents such as vitamin $\mathrm{C}$, vitamin $\mathrm{E}$, beta-carotene, and zinc ameliorated AMD [89]. Moreover, the RPE layer in the human eye is located between the photoreceptor which is prone to lightinduced oxidative damage and the choroid that has a high supply of oxygen [90]. The phagocytosis process by RPE also generates a strong load of free radicals that make RPE more susceptible to oxidative stress. In normal condition, the RPE is capable of removing the free radicals with its own antioxidative mechanism. However, as we age the ability of RPE to compensate the oxidative damage reduces, which leads to series of events that contribute to macular degeneration in the elderly population. One of the reasons for the disruption in the phagocytosis of the POS by RPE is due to the accumulation of lipid and lipid peroxidation products. The presence of drusen deposits is known to increase the risk of progression of AMD. The human drusen accymulate in the extracellular sub-space and consist of lipids, proteins, cellular debris and oxidized lipids. The lipids in the RPE originate primarily from the photoreceptors that are phagocytosed, and these are accumulated with age in Bruch's membrane known as basal linear deposits. The linear deposits are considered as a precursor of drusen formation [91, 92]. The

immunohistochemistry of AMD specimen has shown the staining of oxidized lipids in the Bruch's membrane and basal deposits [93].

Various mass spectrometry based proteomic studies have shown the presence of iron and myeloperoxidase which are highly potent factors that can trigger an oxidative process on proteins and lipids that are accumulated in Bruch's membrane. The lowdensity lipoprotein (LDL) present in a pro-oxidant tissue environment is then oxidized to form oxLDL in which both lipids and proteins become oxidatively modified [94]. The oxLDL is a mixture of a heterogeneous group of macromolecular complexes that consist of various classes of oxidized lipids associated with native or oxidized apolipoprotein B 
Table 3-1. Genes identified to be associated with AMD.

\begin{tabular}{lc}
\hline \multicolumn{1}{c}{ Biological Pathways } & Genes \\
\hline The complement pathway and immune response & CFH, C2/CFB, CFI, C3 and C9 \\
Lipid Transport & APOE,LIPC,CETP,BAIAP2L2 \\
Cell survival/DNA repair/Apoptosis/ Stress response & ARMS2, RAD51B, TNFRSF10A \\
Angiogenesis & VEGFA, TGFBR1, ADAMTS9 \\
ECM remodeling & COL8A1, COL10A1, TIMP3, \\
\hline
\end{tabular}


(apoB). OxLDL bears lipid peroxidation-derived structural moieties called oxidationspecific epitopes (OSEs) which enable recognition of oxLDL by cellular and soluble pattern recognition receptors (PRRs). The PRRs mediate clearance of oxLDL as part of a protective response by the innate immune system to maintain cellular homeostasis [95].

AMD lesion formation has been hypothesized to be similar to the mechanism of formation of atherosclerosis lesions. Both diseases hold common underlying defect where there is deposition of lipids. Both the eye and the arteries are unable to remove the cholesterol deposits effectively. The "response to retention" hypothesis that is widely supported for atherosclerosis states that lipoprotein particles enter the intima of the arteries and bind to specific proteoglycans [96]. These retained lipoproteins are oxidized to oxLDL which is then internalized by macrophages via scavenger receptors mainly CD36 and SR class A (SR-A). This leads to the formation of foam cells that induce the cascade of molecular events that eventually lead to the formation of atherosclerosis plaques. Since various groups have already demonstrated the presence of cholesterol, lipids, and apo-lipoproteins in Bruch's membrane, retention of these lipoproteins in the Bruch's membrane and their oxidative modification is postulated to initiate the cascade of events that brings pathophysiological complications in the eye [97].

\section{Scavenger Receptors of OxLDL}

Scavenger receptors are a group of receptors that are structurally diverse and can be membrane bound or soluble. These receptors are known to identify macromolecules, the modified low-density lipoprotein (either by acetylation and oxidation), and various pathogens, damage-associated molecular patterns (DAMPs) [98] and pathogen-associated molecular patterns (PAMPs)[99]. The role of scavenger receptors in the uptake of oxidized lipids and contribution to the formation of plaques in atherosclerosis lesions is well accepted [100]. There are various scavenger receptors, e.g., CD36, SRB1, and SRBII, present in the eye that are responsible for oxLDL uptake [101]. In the rest of the chapter, I would discuss the two scavenger receptors CD36 and CD5L/AIM that are the main focus of our study.

CD36 is $80 \mathrm{kD}$, a transmembrane protein which is extensively glycosylated, found in a variety of epithelial and endothelial cells including platelets, immune cells, adipocyte, monocyte, retinal and mammary epithelial cells [102]. Structurally, CD36 has both the $\mathrm{N}$ and $\mathrm{C}$-termini located in the cytoplasm and a larger extracellular loop with two transmembrane domains [103]. CD36 binds to many ligands long chain fatty acid, oxidized phospholipids, and has been implicated in diseases such as malaria, thrombosis, and atherosclerosis [104-106]. The extracellular domain at amino terminal is known to contain a binding pocket for oxidized lipids and fatty acids, phospholipids, erythrocytes infected with Plasmodium falciparum. The fatty acids bind at the lysine 164 in the fatty acid binding site, which overlaps with oxLDL binding site near the 155-183 sequence [107], initiating its uptake [108]. Various studies have shown that this binding can be blocked by the use of small molecule sulfo-N-succinimidyl oleate (SSO) which binds irreversibly to the lysine 164 in the CD36 binding pocket blocking the transport of 
oxidized lipids and fatty acids $[109,110]$. CD36 is extensively studied in atherosclerosis where the macrophages expressing CD36 binds and uptakes oxLDL, initiating the formation of atherosclerosis lesions [111]. CD36 is a major receptor for oxidized lipids in the eye, especially oxidized phospholipids. In the eye, CD36 is known to recognize shed photoreceptor outer segments [112, 113], oxidatively modified lipoproteins [114]. It has been shown that oxLDL is readily internalized by ARPE-19 cells via CD36 [115]. Moreover, a study in mice has shown that the uptake of oxLDL by RPE is CD36 dependent [114] which suggests that CD36 plays an important role in clearing the oxidized lipids from Bruch's membrane.

The other scavenger receptor of interest is CD5L/AIM which is also known as apoptosis inhibitor of macrophage. It is a $45 \mathrm{kD}$ secreted scavenger receptor which is identified as an apoptosis inhibitor that supports macrophage survival against apoptosisinducing stimuli [116]. CD5L/AIM circulates in the blood and is abundant around 5 $\mu \mathrm{g} / \mathrm{ml}$ in humans and around $2 \mu \mathrm{g} / \mathrm{ml}$ in mice [117]. In tissue macrophages, the transcription of CD5L/AIM is mediated by nuclear receptors LXR and RXR which protects macrophages from apoptosis [118]. In blood, the AIM proteins associate with IgM pentamers that protects the renal secretion of protein [119].

CD5L is known to play a key role in atherosclerosis. CD5L/AIM is also known to be involved in the foam cells formation in macrophages [120]. Foam cells are fat laden macrophages that serve as hallmark of lesion formation in early stage of atherosclerosis. The uncontrolled uptake of modified LDL via scavenger receptors, impairment of cholesterol release mechanism and upregulation of esterification are some of the mechanism that triggers the formation of macrophage foam cells. In atherosclerosis lesions, CD5L/AIM promotes M2 macrophage polarization. It has also been shown that CD5L/AIM is one of the critical factors that protects the macrophages from oxLDL mediated apoptosis [116]. Furthermore, recent studies on macrophages demonstrated the involvement of CD5L/AIM in uptake of oxLDL via membrane bound CD36 scavenger receptor. However, the presence of $\mathrm{CD} 5 \mathrm{~L} / \mathrm{AIM}$ in the retina and its contribution to oxLDL uptake is not known. Recently our group found that the sera of AMD patients have elevated CD5L/AIM circulating levels [121]. With these background information on the macrophages, and recent exciting findings from our lab provided us following research questions, a) to understand the significance of elevated CD5L/AIM autoantibodies in AMD patients, b) to elucidate the role of CD5L/AIM in uptake of oxLDL by RPE.

To answer above mentioned research questions, we set following central hypothesis, oxidized low density lipoprotein (oxLDL) is internalized by the RPE through the action of scavenger receptors CD36 and CD5L/AIM, and induces a cytoprotective molecular response in the RPE. To test this hypothesis we developed two specific aim for this study i) the transmembrane scavenger receptor CD36 binds oxLDL on the surface of RPE cells and mediates oxLDL uptake by the cells; ii) the RPE express and secrete the soluble scavenger receptor CD5L/AIM. We further postulate that CD5L/AIM present in the extracellular space binds oxLDL and facilitates its uptake (clearance) by the RPE, iii) the oxLDL uptake induces molecular response involving alterations of diverse pathways 
and networks. To fully capture this response, we will use global-scale, discovery-based approaches to characterize oxLDL-induced alterations in the RPE transcriptome and proteome.

\section{Materials and Methods}

The immortalized human retinal pigment epithelium cells ARPE-19 were purchased from ATCC (Manassas, USA) and maintained in DMEM-F12 medium (ATCC, Manassas, USA) containing $2 \mathrm{mM}$ L-glutamine supplemented with $10 \%$ fetal bovine serum (ATCC, Manassas, USA) $100 \mu \mathrm{g} / \mathrm{ml}$ antibiotic (Primocin, Invitrogen) in a humidified atmosphere with $5 \% \mathrm{CO} 2$ at $37{ }^{\circ} \mathrm{C}$. Primary human RPE cells, isolated postmortem as described $[122,123]$ were also obtained. Cells were grown to confluency in cell culture medium according to the same experimental protocol as ARPE-19 cells Exclusion criteria for human donor eyes included known retinal dystrophies, diabetic retinopathy, uveitis, trauma, prior retinal laser treatment, or other proliferative or degenerative retinal diseases. Primary cultures within the first ten passages were used. Antibody for CD5L (CD5L Antibody (F-1): sc-514283), GAPDH (GAPDH Antibody (0411): sc-47724) was purchased from Santa Cruz (Dallas, Texas). IR-Dye labeled secondary antibodies (1 : 15000 dilution; LiCor, Lincoln, Nebraska, USA) were used to detect primary antibodies.

\section{OxLDL Uptake by ARPE-19 Cells}

ARPE-19 cells were grown in the coverslip. Cells were put on SFM for 24 hours before treating the cells with different concentration of DiI-oxLDL. 0.1, 0.5, 1, 2.5, 5, $10 \mathrm{ug} / \mathrm{ml}$ of oxLDL. After $5 \mathrm{~h}$, the excess DiI-oxLDL was washed with PBS, fixed and coverslips were mounted on the glass slide. The images were taken with confocal microscopy. Image J software was used to quantify the red fluorescence in the cells.

\section{Western Blot Analysis}

ARPE-19 cells were lysed, placed in reducing buffer containing $\beta$ mercaptoethanol and heated at $95^{\circ} \mathrm{C}$ for $5 \mathrm{~min}$. Samples were then run in by SDSpolyacrylamide gel electrophoresis on BioRad (Hercules, California, USA). 15\% gradient gels, and then transferred to nitrocellulose membrane using transblotter. The membranes were blocked in LiCor blocking buffer for $1 \mathrm{~h}$ at room temperature and probed with primary antibody in $0.1 \%$ Tween LiCor blocking buffer overnight at $4^{\circ} \mathrm{C}$.

The membranes were washed three times for 10 min each in $0.01 \%$ Tween

phosphate buffer solution then probed with IR-Dye labeled secondary antibodies in $0.1 \%$ Tween, $0.01 \%$ SDS LiCor blocking buffer for $1 \mathrm{~h}$ at room temperature. Washes were repeated after secondary labeling, washing three times for $10 \mathrm{~min}$ in Tween phosphate 
buffer solution and then placed in water. The blots were scanned using the LiCor laserbased image detection method.

\section{End-Point PCR for CD5L Determination}

ARPE-19 cells were grown to confluency according to above mentioned conditions. Total RNA was isolated using the Qiagen RNA extraction kit with DNAse treatment according to the manufacturer's instructions (Santa Clara, CA). The total RNA was quantified by Nano Drop-ND 1000. The PCR amplification reaction (40 cycles) was performed using the following conditions: initial denaturation at $95{ }^{\circ} \mathrm{C}$ for $5 \mathrm{~min}, 95{ }^{\circ} \mathrm{C}$ for $30 \mathrm{~s}, 65^{\circ} \mathrm{C}$ for $30 \mathrm{~s}$ and $72{ }^{\circ} \mathrm{C}$ for $1 \mathrm{~min}$. The following primer pairs were used: $5^{\prime}-$ TGG GAC ATT AAG GAC GTG GC -3' (forward) and 5'- CGG TCT CTG AAG GAG GGA GA -3' (reverse) for CD5L/AIM, and 5'- ACC ACA GTC CAT GCC ATC AC -3' (forward) and 5'- TCC ACC ACC CTG TTG CTG TA -3' (reverse) for GAPDH. The PCR product was analyzed by agarose gel electrophoresis. After gel electrophoresis, the CD5L/AIM DNA band was cut from the gel and analyzed by DNA sequencing to confirm the expected CD5L/AIM transcript

\section{CD5L/AIM Binding Assay}

$5 \mathrm{ug} / \mathrm{ml}$ of rhCD5L/AIM was coated on a 96 well plate overnight at $4{ }^{\circ} \mathrm{C}$. The plate was then washed with fat free BSA for three times to remove the unbound rhCD5L/AIM. Next, DiI-oxLDL was added in triplicate in different concentrations for 4 hours. The plate was then washed for two hours with fat free BSA. The DiI fluorescence was measured with the Cytation5 fluorescent reader with excitation wavelength 514 and emission 550. The binding constant was calculated using a graph pad prism.

\section{Cellular Uptake Assays of OxLDL by Confocal Microscopy and Flow Cytometry}

ARPE-19 cells were grown in a 24 well plate until confluency. Cells were put on SFM for $24 \mathrm{hrs}$ before treating them with $10 \mathrm{ug} / \mathrm{ml}$ of DiI-oxLDL. After 5 hours, the excess DiI-oxLDL was washed with PBS, the cells were trypsinized and collected on PBS, and the uptake was analyzed with flow cytometer. For confocal microscopyARPE19 cells were grown in a 24 well plate until confluency. Cells were put on SFM for $24 \mathrm{~h}$ before treating them with $10 \mathrm{ug} / \mathrm{ml}$ of oxLDL. After $5 \mathrm{~h}$, the excess DiI-oxLDL was washed with PBS, the cells were fixed, and the uptake was analyzed with a confocal microscope.

\section{Blocking of CD36 Receptor for OxLDL Uptake Studies}

ARPE-19 cells were grown in a coverslip. Cells were put on SFM for $24 \mathrm{~h}$ before pre-treating the cells with $100 \mu \mathrm{M}$ of SSO for one hour. After the SSO treatment, the 
cells were treated with $10 \mathrm{ug} / \mathrm{ml}$ of DiI-oxLDL for $5 \mathrm{~h}$. Next, the excess DiI-oxLDL was washed with PBS, fixed and coverslips were mounted on the glass slide. The images were taken with confocal microscopy. Image J software was used to quantify the red fluorescence in the cells.

\section{qPCR Analysis for OxLDL Mediated Gene Expression}

ARPE-19 cells were grown in a 6 well plate until confluence. Cells were put on SFM for $24 \mathrm{~h}$ before the treatment with $100 \mathrm{ug} / \mathrm{ml}$ of oxLDL/LDL for $4 / 24 \mathrm{~h}$. The cells were then harvested for RNA extraction. qPCR analysis on genes of interest was performed using SYBR green assay using following primers: for LXRalpha, CCC CAT GAC CGA CTG ATG TT (forward), CGG AGG CTC ACC AGT TTC ATT (reverse); for CD36, GAG AAC TGT TAT GGG GCT AT (forward), TTC AAC TGG AGA GGC AAA GG(reverse); for G3PDH, ACC ACA GTC CAC GCC ATC AC (forward), TCC ACC ACC CTG TTG CTG TA (reverse); for CD5L GGC ATC GTG TGA GAA CCC AGA G(forward), CTC ACA CGA TGC CCC AGC ATC (reverse).

\section{Induction of CD5L/AIM with LXR-Agonist}

ARPE-19 cells were grown in a 6-well plate until confluence. Cells were put on SFM for $24 \mathrm{~h}$ before the treatment with $\mathrm{ug} / \mathrm{ml}$ of T090 for $24 \mathrm{~h}$. The cells were then harvested for RNA extraction. qPCR analysis on ABCA1 \{GCA CTG AGG AAG ATG CTG AAA (forward), AGT TCC TGG AAG GTC TTG TTC AC (reverse)\} and CD5L/AIM was performed using the SYBR green assay.

\section{Cellular Uptake Assay of OxLDL by Flow Cytometry Using rhCD5L/AIM}

ARPE-19 cells were grown in a 24 well plate until confluency. The cells were put on SFM for $24 \mathrm{~h}$ before treating the cells with DiI-oxLDL and rhCD5L/AIM. Cells were then treated with $0.1 \mathrm{ug} / \mathrm{ml}$ of DiI-oxLDL and $10 \mathrm{ug} / \mathrm{ml}$ of rhCD5L/AIM. After 5 hours, the excess DiI-oxLDL and rhCD5L/AIM was washed with PBS, the cells were trypsinized and collected on PBS, and the uptake was analyzed with flow cytometer.

\section{Statistical Analysis}

Quantitative data are presented as mean \pm standard error of the mean (SEM). All measurements were performed on triplicate samples. All experiments were conducted independently at least three times with three or more technical replicates. 


\section{Results and Discussion}

\section{ARPE-19 Cells Uptake OxLDL via CD36}

To test the hypothesis that ARPE-19 cells uptake oxLDL, ARPE-19 cells were treated with DiI-oxLDL for $5 \mathrm{~h}$. The excess oxLDL after the treatment was removed by washing. The cells were then fixed with PFA and then confocal images were taken (Figure 3-2A, B) by confocal microscopy. For flow cytometry, the cells were washed, trypsinized and the uptake was measured by flow cytometer (Figure 3-2C). For this experiment, DiI labeled oxLDL was used, and ARPE-19 cells were treated with $10 \mathrm{ug} / \mathrm{ml}$ of DiI-oxLDL. Our results demonstrate that cultured RPE cells internalize oxLDL thus confirming similar findings by others $[115,124]$.

To determine the role of the scavenger receptor CD36 in the uptake of oxLDL by ARPE-19 cells, prior to exposure to oxLDLwe treated the ARPE-19 cells with SSO which is a small molecule known to block CD36. The treatment of SSO for $1 \mathrm{~h}$ before the oxLDL treatment significantly reduced the uptake of oxLDL in ARPE-19 cells (Figure 3-2B). The flow cytometry analysis of both the treated and untreated cells also showed similar result (Figure 3-2D). The results indicate that CD36 is the major receptors for oxLDL uptake by the ARPE-19 cells.

\section{ARPE-19 Cells Express CD5L/AIM}

The presence of CD36 in ARPE-19 cells was well-documented, however, the presence of CD5L/AIM was unknown until this study. We established the presence of CD5L/AIM in ARPE-19 cells by three different assays. It is noteworthy to mention that with all the experiments involving the CD5L/AIM, the cells were serum deprived at least for $24 \mathrm{~h}$ before any treatments to eliminate the cross contamination of CD5L/AIM from bovine serum. The cellular localization of CD5L/AIM in ARPE-19 cells was confirmed by immunofluorescence assay. CD5L/AIM was present in the ARPE-19 cells both in cytoplasm and nucleus (Figure 3-3A, B). End-point PCR analysis was performed to validate the presence of CD5L/AIM transcript in ARPE-19 (Figure 3-3C). Moreover, CD5L/AIM protein level expression in both the ARPE-19 and primary RPE was determined by western blot analysis. Both the cell lines showed the presence of CD5L/AIM and the retinal pigment epithelial cell marker RPE65 (Figure 3-3D). Besides, the use of blocking peptide for the sequence of CD5L/AIM antibody diminished the CD5L/AIM detection in western blot. 

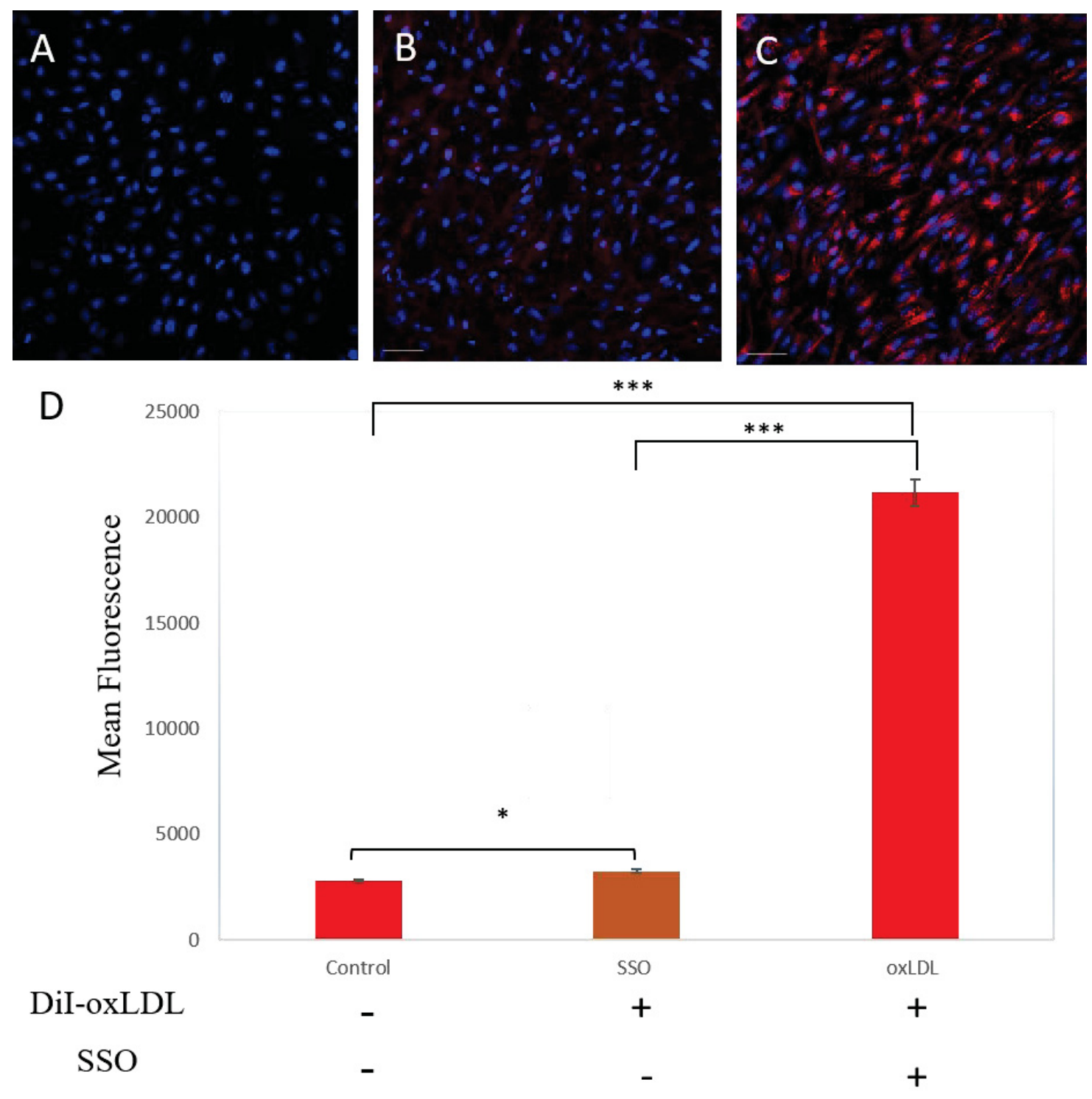

Figure 3-2. oxLDL uptake by ARPE-19 cells.

(A) Control (no treatment), (B) SSO treatment with DiI-oxLDL, (C) DiI-oxLDL only. Confocal Image: The ARPE-19 cells when treated with a small molecule SSO known to irreversibly block the CD36 receptors for one hour before adding the DiI labeled oxLDL for $5 \mathrm{~h}$. The excess DiI-oxLDL was washed with PBS the cells were then fixed with 4\% PFA and imaged with confocal microscope.(D) The blocking of CD36 caused significant decrease in oxLDL uptake Flow cytometry: ARPE-19 cells were treated with DiIoxLDL for $5 \mathrm{~h}$.The excess DiI-oxLDL was washed with PBS the cells were trypsinized and collected in PBS. Fluorescence was measured by flow cytometry. Blocking of oxLDL uptake by SSO: The ARPE-19 cells when treated with a small molecule SSO known to irreversibly block the CD36 receptors for one hour before adding the DiI labeled oxLDL.. Mean fluorescence intensity ratio in cells scored in 10,000 cells were calculated by flow cytometer with three technical replicates from 3 independent experiments. $* \mathrm{P} \leq 0.05 ; * * * \mathrm{P} \leq 0.001$ 

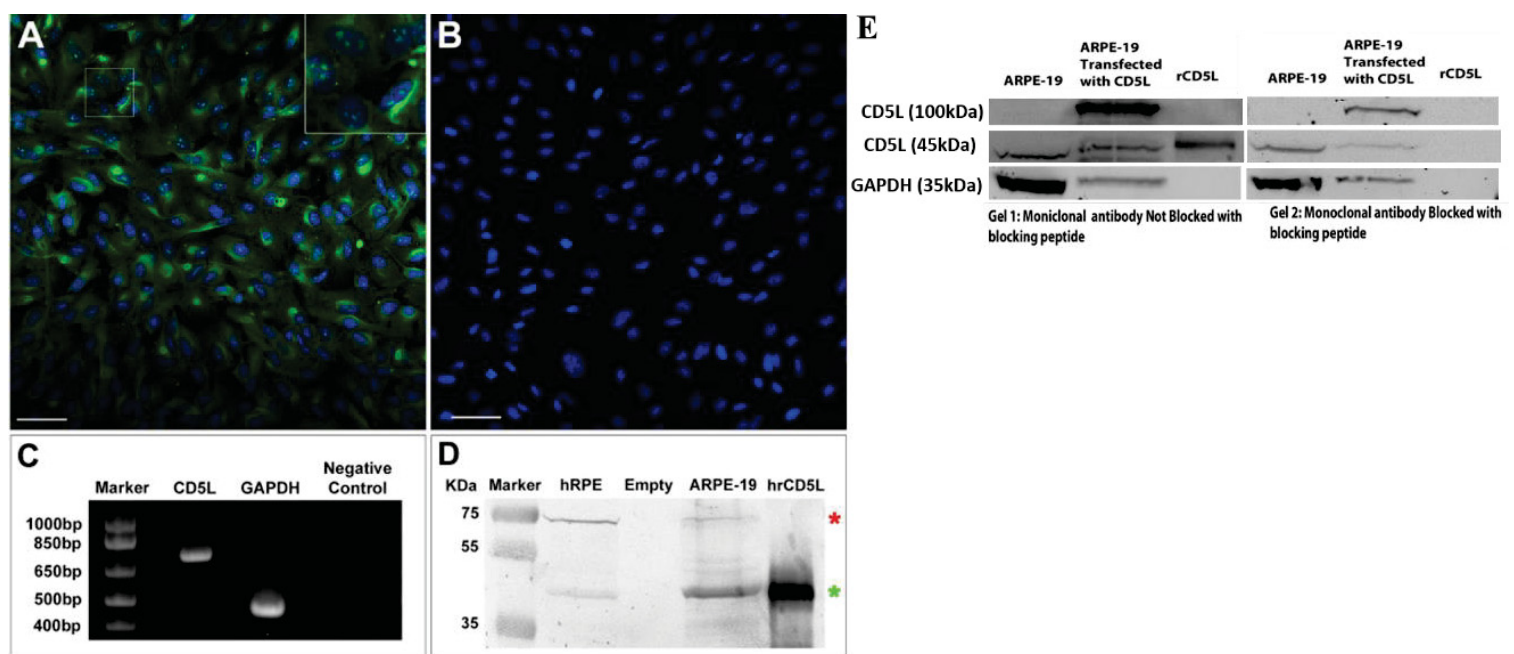

Figure 3-3. Expression of CD5L/AIM/AIM in the retinal pigment epithelium. (A) Confocal microscopy on immortalized human ARPE-19 cells. CD5L/AIM immunofluorescence is shown in green (blue, DAPI nuclear stain). Note the diffuse granular cytoplasmic reactivity seen in the ARPE-19 cells and the more discrete, clumplike nuclear localization of the reactivity (see also magnified view in the inset in the top right hand corner, corresponding to the cropped area in A). (B) Negative control for ARPE-19 cells. Bar in A and B $=50 \mu \mathrm{m}$. (C) Results of end-point PCR demonstrate expression of the expected CD5L/AIM/AIM transcript in ARPE-19 cells, which was further confirmed by sequencing of the band (GAPDH, positive control; empty lane, negative control). (D) Results of Western blots performed on cell culture lysates of a primary human RPE cell line (hRPE) and an immortalized ARPE-19 cell line (green asterisk: CD5L/AIM/AIM band), confirming expression of CD5L/AIM/AIM also in both cell lines (red asterisk: RPE65 control band; last lane: human recombinant CD5L/AIM/AIM, positive control). (E) Confirmation of CD5L/AIM in ARPE-19 cells by using blocking peptide for the antibody against CD5L/AIM.

Note: Panels A,B,C,D reprinted with permission from Elsevier Publications. Iannaccone, A., et al., Retinal pigment epithelium and microglia express the CD5 antigen-like protein, a novel autoantigen in age-related macular degeneration. Experimental Eye Research, 2017. 155 (121) 


\section{Does CD5L/AIM Bind to OxLDL?}

Interaction of CD36 with oxLDL and its uptake in ARPE-19 was studied in various studies $[114,125]$. Since we wanted to explore the involvement of CD5L/AIM interaction with oxLDL and its uptake, we performed an ELISA assay where we coated the plate with rh CD5L/AIM and evaluated the binding of DiI-oxLDL with the rhCD5L/AIM. The binding affinity was calculated to be $13.6 \mathrm{kD}$ by graph pad prism using 'one site-total and non specific binding' equation (Figure 3-4A). We also transfected the ARPE-19 cells with CD5L/AIM and then treated the transfected cells with DiI-oxLDL. The confocal images show the co-localization of DiI-oxLDL particles (yellow dots) due to DiI-oxLDL (red) with CD5L/AIM (green) (Figure 3-4B).

\section{CD5L/AIM and CD36 Co-localize in the Presence of OxLDL}

When ARPE-19 cells were treated with $100 \mathrm{ug} / \mathrm{ml}$ of oxLDL for $4 \mathrm{~h}$, colocalization was observed between CD5L/AIM and CD36. This effect was not seen in the cells that were not treated with oxLDL. ARPE-19 cells were treated with $100 \mathrm{ug} / \mathrm{ml}$ of oxLDL for $4 \mathrm{~h}$ and then the cells were washed and fixed with PFA. The cells were then incubated overnight with CD36 and CD5L/AIM antibody and then treated with suitable secondary antibody. The images were taken with confocal microscopy. The colocalization of CD5L/AIM and CD36 was seen as yellowish orange dots in oxLDL treated cells (Figure 3-5).

\section{Effects of Anti-CD5L/AIM Antibody on OxLDL Uptake}

To evaluate the effects of anti-CD5L/AIM antibody on the uptake of oxLDL by ARPE-19 cells, the cells were pre-treated with anti-CD5L/AIM antibody and IgG2A isotype control for $1 \mathrm{~h}$ at $37{ }^{\circ} \mathrm{C}$. Then the cells were re-incubated for $5 \mathrm{~h}$ with DiI-oxLDL and anti-CD5L/AIM antibody and IgG2A for $5 \mathrm{~h}$. After $5 \mathrm{~h}$, the cells were washed and then the uptake was measured by flow cytometry. The oxLDL uptake due to antiCD5L/AIM treatments prior to and during the oxLDL treatment by ARPE-19 cells did not show significant change (Figure 3-6).

\section{CD5L/AIM, CD36 Expression Changes Due to OxLDL Treatment}

Towards this aim, we treated the ARPE-19 cells with $100 \mathrm{ug} / \mathrm{ml}$ for different time points, $4 \mathrm{~h}$ and $24 \mathrm{~h}$. There was no significant difference between the expression of both the CD5L/AIM and CD36 at 4h. and 24h due to oxLDL treatment when normalized against housekeeping gene GAPDH (Figure 3-7). 
A

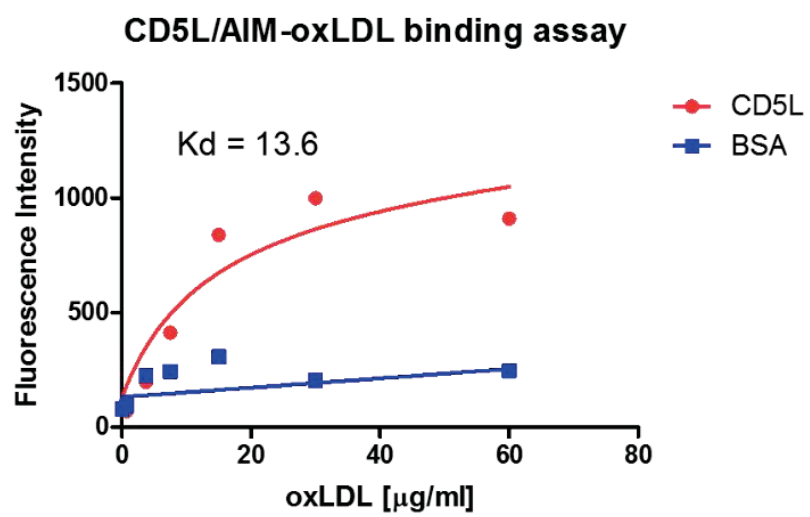

B

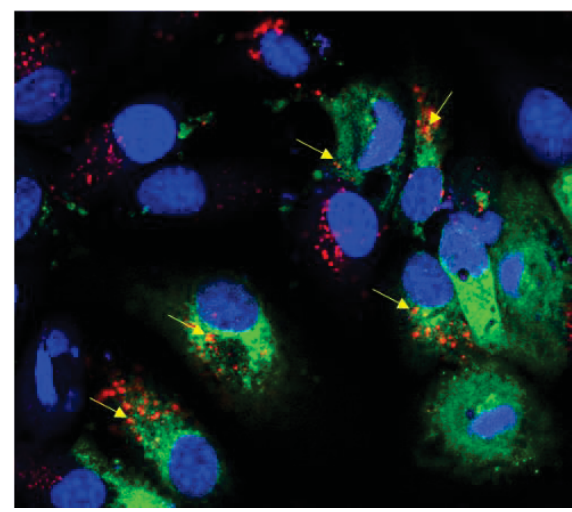

Figure 3-4. CD5L/AIM-OxLDL binding assay.

(A) CD5L/AIM binding Assay (B) ARPE-19 cells were transfected with CD5L/AIM and then treated with DiI-oxLDL for 4 hours. The cells were washed, fixed and then confocal images were taken. The z-stack images show the co-localization of CD5L/AIM and oxLDL as indicated by yellow arrows. 

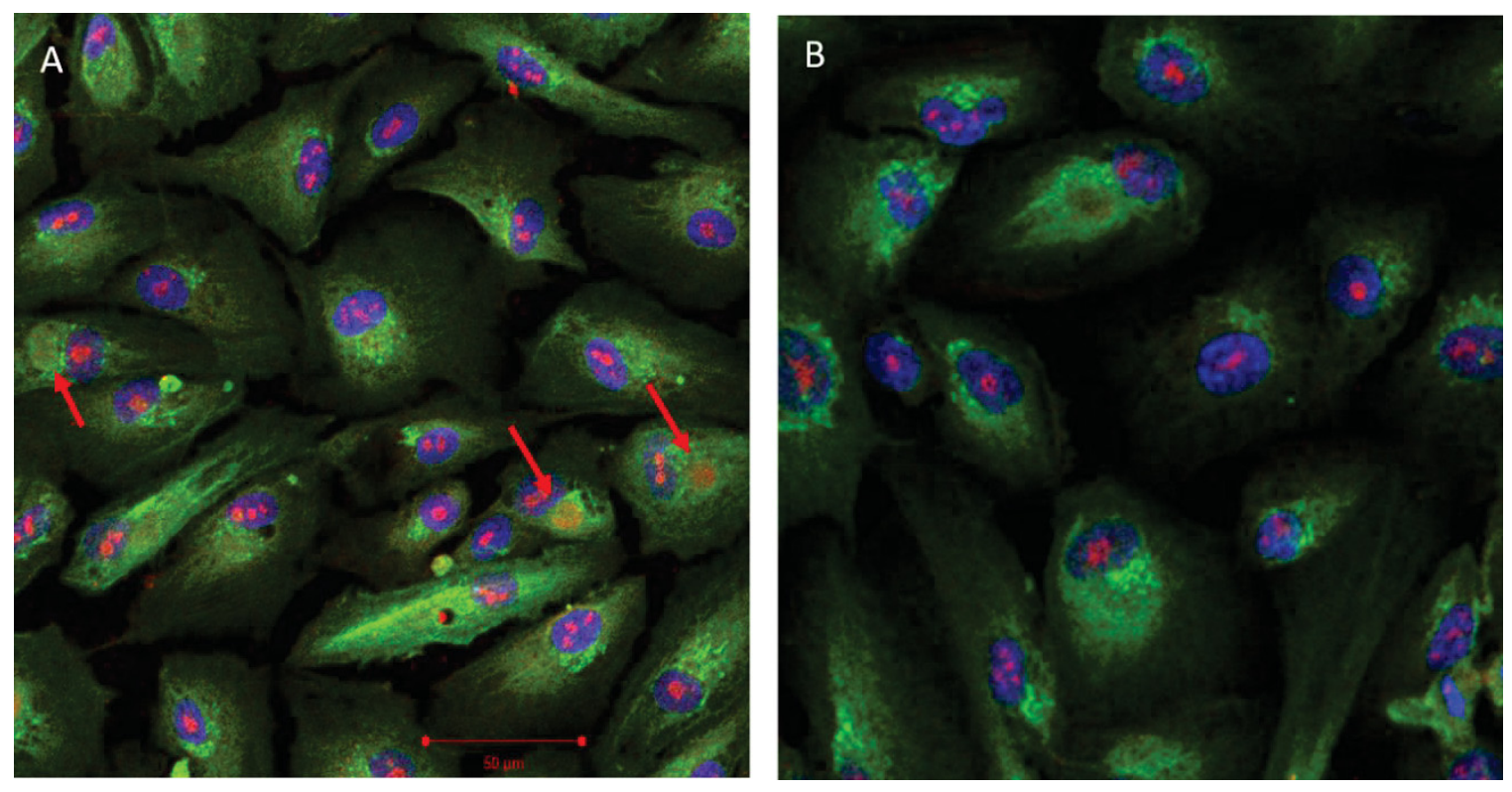

Figure 3-5. Co-localization of CD36 and CD5L/AIM.

(A) shows the oxLDL treated cells. (B) shows the cells that were not treated with oxLDL. The ARPE-19 cells were grown on coverslip until confluence. The cells were treated with $100 \mathrm{ug} / \mathrm{ml}$ of oxLDL for 5 hours after serum starving overnight. The cells were then fixed and stained for CD36 (green) and CD5L/AIM(red) and DAPI (blue). The overlap is indicated by red arrows The images were taken by confocal microscopy. At least three biological replicate experiments were performed each with three technical replicates. 


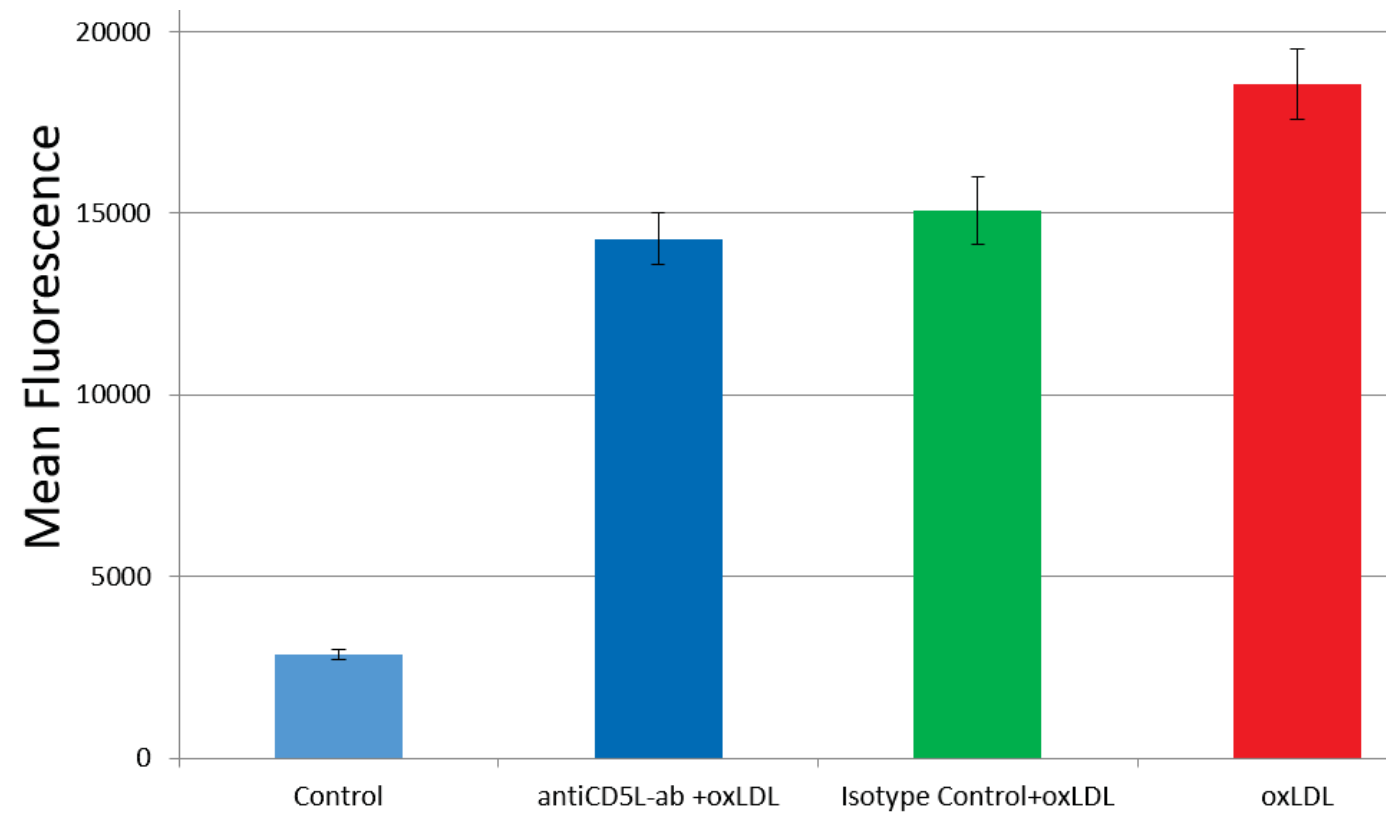

Figure 3-6. Effects of anti-CD5L/AIM antibody on the OxLDL uptake.

ARPE-19 cells were grown to confluency and serum starved for 24 hours before treating them with anti-CD5L/AIM ab and IgG (isotype control). The ARPE-19 cells were then co-treated with DiI-oxLDL for 5 hours. There was a decrease in uptake due to antiCD5L/AIM antibody treatment, but it was not significant than the decrease due to isotype control. At least three biological replicate experiments were performed each with three technical replicates. 


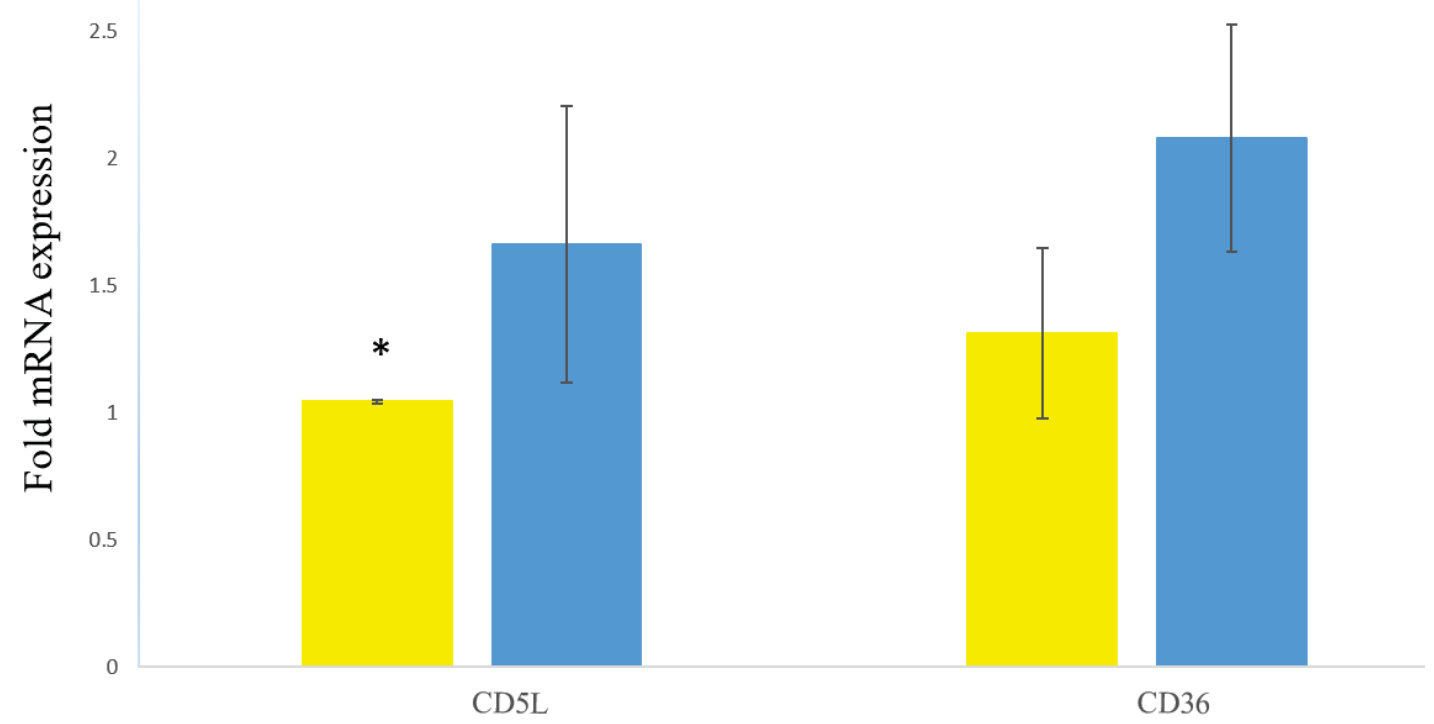

Figure 3-7. Effects of OxLDL on scavenger receptors.

The ARPE-19 cells were treated with $100 \mathrm{ug} / \mathrm{ml}$ of oxLDL for 4 and $24 \mathrm{~h}$. The changes in the transcript of CD36, CD5L/AIM, were evaluated by SYBR-GREEN qPCR analysis. At least three biological replicate experiments were performed each with six technical replicates. ${ }^{*} \mathrm{p}<0.05 ; * *<0.01 ; * * * \mathrm{p}<0.001$. 


\section{Effect of LXR Agonist on Expression of CD5L/AIM}

Since no significant blocking of oxLDL uptake was seen by the use of antiCD5L/AIM antibody, we wanted to investigate the effect of LXR agonist on the expression of CD5L/AIM. To demonstrate this effect, the ARPE-19 cells were grown to confluency and treated with T090 (LXR-agonist) for 24 hours and the expression of ABCA1 and CD5L/AIM was examined via sybr-green qPCR. The LXR agonist T090 is known to induce the expression of cholesterol efflux regulatory protein ABCA1. The use of LXR-agonist did not induce the expression of CD5L in ARPE-19 cells when normalized with the housekeeping gene GAPDH. However, significant induction was observed for ABCA1 gene (Figure 3-8).

\section{Recombinant CD5L/AIM (rh-CD5L/AIM) Effects the OxLDL Uptake by ARPE-19 Cells}

Since no significant blocking of oxLDL uptake was seen by the use of antiCD5L/AIM antibody, we wanted to investigate the effect of recombinant CD5L/AIM (rh CD5L/AIM) in oxLD1 uptake by the cell lines. To this end, the cells were treated with 10 $\mu \mathrm{g} / \mathrm{ml}$ of rh-CD5L/AIM, and BSA as control along with $0.1 \mu \mathrm{g} / \mathrm{mL}$ DiI-oxLDL for $5 \mathrm{~h}$ at $37^{\circ} \mathrm{C}$. Next, the cells were washed and collected for flow cytometry analysis. Our result showed that the addition of rh-CD5L/AIM along with DiI-oxLDL significantly decreased the amount of DiI-oxLDL within the ARPE-19 cells; (p-value 8.5499E-06) . However, control protein i.e. fat free BSA did not show significant decrease in mean fluorescence of oxLDL as shown in Figure 3-9.

Age-related macular degeneration is a complex multifactorial disease and formation of drusen is considered as the hallmark of the disease. Drusen is present between the retinal pigment epithelium and the Bruch's membrane that consists of oxidized lipids and proteins. Moreover, these oxidized lipids accumulate preferentially in the macula of human eyes over age [126]. Since, RPE cells are the major player of lipid metabolism in the retina [127], the accumulation of lipids below RPE points towards the improper handling of lipids by these cells as we age. Also, AMD is similar to atherosclerosis where the retained lipoproteins are oxidized that induce a cascade of molecular events that eventually leads to the formation of atherosclerosis plaques. Hence in the similar manner, the accumulation of drusen containing oxidized lipids contributes a major factor in the development of AMD.

It is well-accepted that the accumulated oxidized lipids play a crucial role in the development of AMD. In addition, the retina is very vulnerable to oxidative stress. Furthermore, these oxidized lipids are identified by scavenger receptors present in the RPE cells which aid in clearance of oxidized lipids by these cells. With this important information generated by various studies, we investigated the effect of oxLDL on ARPE19 cells and elucidate the role of scavenger receptors CD36 and CD5L/AIM in retinal homeostasis. Treatment of ARPE-19 cells with 100ug/ml of oxLDL for four hours did 


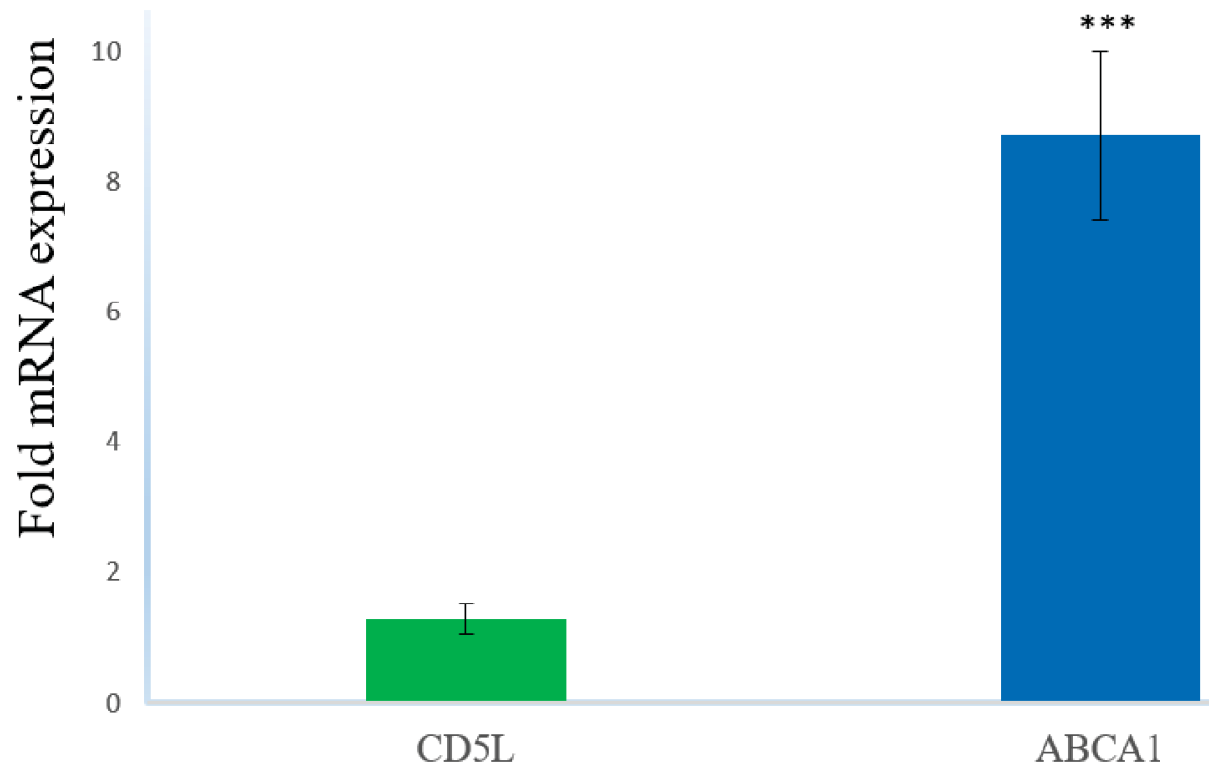

Figure 3-8. Effect of T090 on ARPE-19 cells.

ARPE-19 cells were treated with T090. The expression of CD5L/AIM and ABCA1 was measured by qPCR.There was significant induction of ABCA1 gene due to LXR-agonist (T090) however no significant increase in CD5L expression was seen in our experiments. 


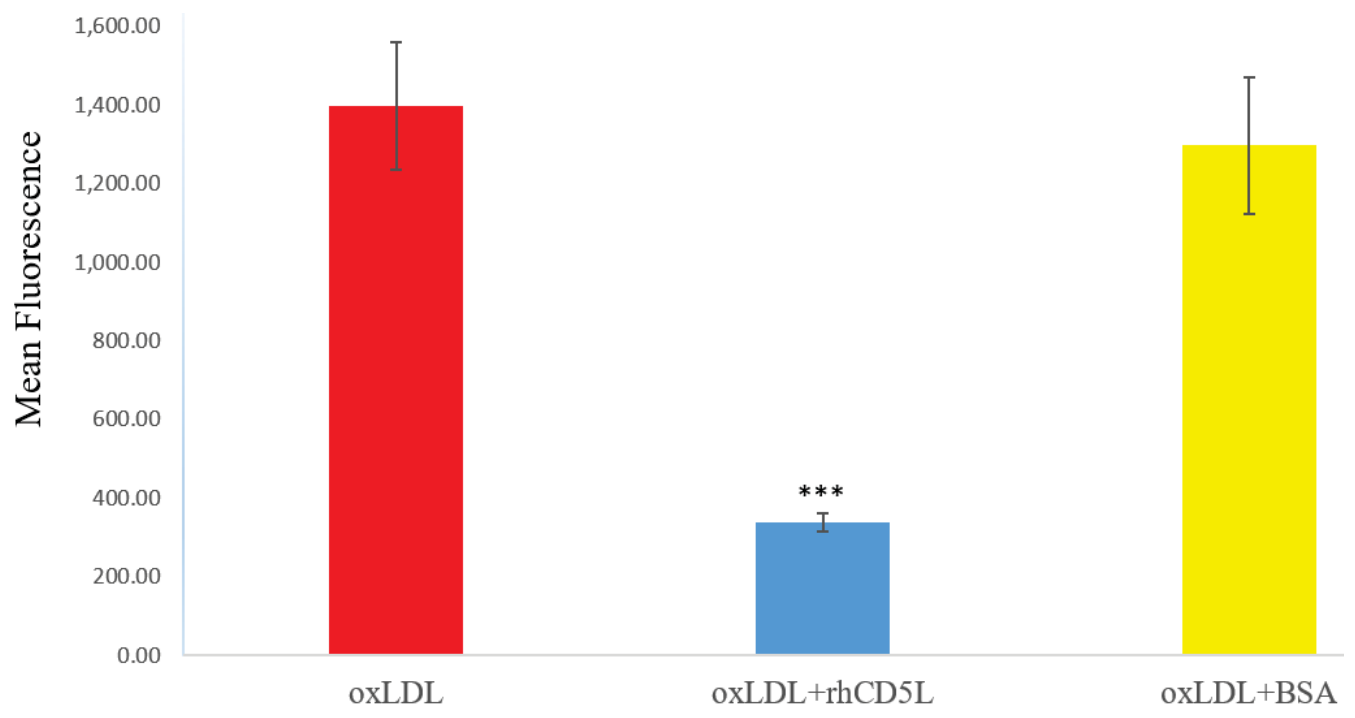

Figure 3-9. Effect of rh-CD5L/AIM on OxLDL uptake.

ARPE-19 cells were grown to confluency and serum starved for 24 hours before treating them with rh-CD5L/AIM and BSA (control protein) and DiI-oxLDL for 5 hours. There was a significant decrease in mean fluorescence for DiI-oxLDL due to rh-CD5L/AIM treatment. Figure shows mean fluorescence intensity ratios in 10,000 cells with three technical replicates, from three independent experiments $* \mathrm{P} \leq 0.05 ; * * * \mathrm{P} \leq 0.001$. 
not induce cell death. In contrast, when the cells were treated for $24 \mathrm{~h}, 20 \%$ of cell death was observed. Since we wanted to observe the role of scavenger receptors and its effect on oxLDL treated ARPE-19 cells, we choose $4 \mathrm{~h}$ time point where there was no cell death.

To elucidate the role of CD36 and CD5L/AIM in oxLDL uptake by ARPE-19 cells, we first demonstrated by confocal microscopy and flow cytometry that the oxLDL is indeed up taken by ARPE-19 cells. Similarly, we demonstrated that ARPE-19 cells uptake oxLDL, which was primarily dependent on CD36. CD36 is a primary scavenger receptor that is expressed in numerous cell types including RPE and other cells of the immune system such as macrophages, microglia, etc. In a recent study on ARPE-19 cells, the use of an anti CD36 antibody (that blocked the CD36 receptor) in the presence of oxLDL has shown concentration dependent decrease in uptake of oxLDL by the cells [125]. In our study, we used a small molecule sulfo-N-succinimidyl oleate (SSO), a potent CD36 binding inhibitor [128]. The pre-treatment of ARPE-19 cells by SSO significantly decreased the uptake of DiI-labeled oxLDL in the cells, thus indicating that CD36 is a major receptor responsible for internalization of oxLDL by RPE.

CD5L/AIM was another scavenger receptor of our interest since our recent study on the autoantibodies expressed in AMD patient sera had shown an increase in CD5L/AIM autoantigen in the diseased population [121]. We also determined the presence of CD5L/AIM in ARPE-19 cells by various independent experiments which provided us a baseline to build up our experiments. Before investigating the role of CD5L/AIM in the uptake of oxLDL, it was important to determine the binding of CD5L/AIM and oxLDL in-vitro. The affinity of oxLDL with rh-CD5L/AIM was reported in a recent study by ELISA [120]. In this study, we demonstrated that CD5L/AIM binds oxLDL in-vitro.. The cells that were transfected with CD5L/AIM (green) and treated with DiI-oxLDL (red) showed co-localization of CD5L/AIM and DiIoxLDL. Moreover, the CD5L/AIM and CD36 were seen to co-localize in the presence of oxLDL. The CD5L/AIM and CD36 axis in the uptake of oxLDL by macrophages has been reported by other groups [120].

CD5L/AIM is known to play a major role in atherosclerosis, a disease which is considered mechanistically close to AMD [129]. Also, it is known to be involved in macrophage survival and foam cell formation [120, 130]. In macrophages, the activation of LXR/RXR nuclear receptor by LXR agonist induces the expression of CD5L/AIM which protected macrophages from bacteria-induced apoptosis $[131,132]$.However, our results did not show the change in CD5L/AIM and CD36 in the transcript or protein level due to $4 \mathrm{~h}$ of oxLDL treatment. The use of anti-CD5L/AIM did not affect the oxLDL uptake by ARPE-19 cells when compared with the isotype control.

Recently published work on acute kidney injury in mice showed that circulating CD5L/AIM helped in the removal of intraluminal debris along with KIM-1 (kidney injury molecule-1) [133]. Since our data on blocking the CD5L/AIM by anti CD5L/AIM antibody present in ARPE-19 cells did not show significant effect on oxLDL clearance 
by the cells, measured as the amount of oxLDL accumulated within the cells, we were interested to see the effect of adding external CD5L/AIM on the oxLDL clearance by the ARPE-19 cells. For this, we used recombinant CD5L/AIM (rh-CD5L/AIM) and incubated the cells with DiI-oxLDL in the presence of rh-CD5L/AIM. Our results showed a significant reduction of intracellular oxLDL accumulation in ARPE-19 cells in the presence of extracellular rh-CD5L/AIM.

\section{Conclusion}

Our results demonstrated a new dynamics on CD5L/AIM on oxLDL uptake that was not seen in macrophages. The reduction in intracellular accumulation of oxLDL due to the presence of extracellular recombinant CD5L/AIM is an interesting phenomenon as it has been recently shown the involvement of CD5L/AIM in autophagy [133]. It would be interesting to know how the CD5L/AIM impacts the oxLDL clearance by the cells. One explanation for our findings is that, CD5L/AIM blocks the uptake of oxLDL and alternative explanation is that CD5L/AIM aids in early intracellular degradation and clearance of oxLDL by ARPE-19 cells. For this, the use of an antibody that could bind to the rh-CD5L/AIM or IgM which is known to bind to the CD5L/AIM could help elucidate the mechanism. Second, a time-course study of oxLDL clearance in the presence of CD5L/AIM encompassing early time points would illuminate whether CD5L/AIM prevents oxLDL uptake or whether CD5L/AIM facilitates intracellular degradation of oxLDL. If CD5L/AIM promotes intracellular clearance of oxLDL, we would expect that at early time points of oxLDL exposure, presence of CD5L/AIM would not cause a major difference in oxLDL accumulation but that over time, there would be a reduction in the accumulation of intracellular oxLDL, as we observed at the single time point examination (after $5 \mathrm{~h}$ exposure) presented here. Finally, the use of autophagy inhibitor along with rh-

CD5L/AIM would validate the role of rh-CD5L/AIM in oxLDL degradation by ARPE-19 cells and help to understand the involvement of CD5L/AIM in autophagy related processes. 


\section{CHAPTER 4. EARLY TRANSCRIPTOME ALTERATIONS INDUCED BY OXLDL IN RETINAL PIGMENT EPITHELIUM CELLS}

\section{Introduction}

Age related macular degeneration (AMD) is the leading cause of legal blindness in the Western world [134]. The pathogenesis of AMD is not fully understood, with age being the major risk factor, some genetic determinants and environmental factors are known to play an important role in the progression of the disease [135-138]. Oxidative stress and inflammation are considered as main events leading to the pathophysiology of the disease [139]. The retinal pigment epithelium (RPE), the principal tissue affected in early stage AMD, is considered a major site of oxidative stress in the retina due to its location: between the photoreceptor outer segments, exposed to photo oxidative light energy, and the choroid with high oxygen content. In this study, we focused on oxidized low-density lipoprotein (oxLDL), which is a major component of drusen and a known inducer of cellular oxidative stress with pro-inflammatory properties [140]. OxLDL generates from low-density lipoprotein (LDL) in a pro-oxidant tissue environment in which lipids and proteins become oxidatively modified [94] It comprises a heterogeneous group of macromolecular complexes consisting of various classes of oxidized lipids associated to native or oxidized apolipoprotein B (apoB). As stated in previous chapter oxidation-specific epitopes (OSEs) enable recognition of oxLDL by cellular and soluble pattern recognition receptors (PRRs) that mediate clearance of oxLDL as part of a protective response by the innate immune system to maintain cellular homeostasis [95]. The response of ARPE-19 cells to oxidative stress induced with various agents has previously been reported. Weigel et al. analyzed the transcriptome alteration in ARPE-19 cells after a $4 \mathrm{~h}$ exposure to various pro-oxidizing agents including $\mathrm{H}_{2} \mathrm{O}_{2}$, 4hydroxynonenal and tert-butylhydroperoxide [141]. Cano et al. performed a transcriptomics analysis of ARPE-19 cells exposed to cigarette smoke extract (CSE) [142]. Similarly to the study presented here, Koller et al. used transcriptomics analysis of RAW 264.7 macrophages after a $4 \mathrm{~h}$ exposure to oxidized phospholipids, which are common constituents of oxLDL [143]. Yamada et al. studied the effects of oxLDL induced oxidative stress in ARPE-19 cells and focused on the cellular response after $48 \mathrm{~h}$ treatment [124]. To obtain a comprehensive outlook of the early transcriptome alterations induced by oxLDL exposure, we performed microarrays gene expression analyses in ARPE-19 cells following a 2 and $4 \mathrm{~h}$ treatment with non-lethal doses of oxLDL. We report that various gene pathways including genes involved in anti-oxidative defense mechanisms, circadian rhythm regulation, and lipid metabolism are induced in a timedependent and selective fashion by oxLDL treatment. Gene expression changes as an early response to oxLDL treatment in RPE cells could help to better understand the early molecular events in the pathogenesis of AMD and decipher the role of oxidative stress to provide new opportunities for better treatment and prevention. 


\section{Materials and Methods}

\section{Cell Culture and RNA Preparation}

The immortalized human retinal pigment epithelium cells ARPE-19 were purchased from ATCC (Manassas, USA) and maintained in DMEM-F12 medium (ATCC, Manassas, USA) containing $2 \mathrm{mM}$ L-glutamine supplemented with $10 \%$ fetal bovine serum (ATCC, Manassas, USA) $100 \mu \mathrm{g} / \mathrm{ml}$ antibiotic (Primocin, Invitrogen) in a humidified atmosphere with $5 \% \mathrm{CO}_{2}$ at $37{ }^{\circ} \mathrm{C}$. For the cytotoxicity assay cells were grown in 96-well plates in serum-containing medium. Before LDL or oxLDL treatment, the cells were grown in serum-free medium for $24 \mathrm{~h}$ followed by addition of oxLDL or LDL $(0-800 \mu \mathrm{g} / \mathrm{ml})$. After $24 \mathrm{~h}$ treatment, $50 \mu \mathrm{l}$ of the medium was transferred to a fresh 96-well flat clear bottom plate for measurement of lactate dehydrogenase (LDH) release with CytoTox 96® according to manufacturer's recommendations. The plates' absorbance at $490 \mathrm{~nm}$ was measured with Bio Tek Synergy 2 fluorescence reader. The EC50 value was calculated with Prism v. 7.0 (GraphPad). All experiments were performed in multiple biological replicates $(n=4)$. OxLDL (TBARS: 29-44 nmoles $\mathrm{MDA} / \mathrm{mg}$ ) and LDL were obtained from Alfa Aesar (Tewksbury, USA). Cells (150,000) were seeded on 6 well plates and grown until confluent. Serum-starved (24 h) ARPE-19 cells were treated with $100 \mu \mathrm{g} / \mathrm{ml}$ of oxLDL and $100 \mu \mathrm{g} / \mathrm{ml}$ of LDL for $2 \mathrm{~h}$ and $4 \mathrm{~h}$, respectively. All experiments were performed in multiple biological replicates $(n=6)$. Total RNA was extracted with the Qiagen RNeasy mini kit after DNAse treatment, and RNA quality and quantity were validated with the Agilent Bio Analyzer microfluidics chip RNA Nano 6000. Samples were stored at $-80{ }^{\circ} \mathrm{C}$ until further use.

\section{Microarray Analysis}

RNA samples were analyzed with an Affymetrix Human Clariom S array using Affymetrix WT Plus Amplification kit according to manufacturer's protocols. Arrays were washed and stained on Affymetrix Fluidics Station 450 and scanned on Affymetrix Scanner GCS3000. Data were normalized using the Affymetrix Expression Console. The DEGs were determined based on fold change (FC) and Benjamini-Hochberg adjusted false discovery rate (FDR). Cutoffs values of $|\mathrm{FC}|>1.5$ and $\mathrm{FDR} \leq 5 \%$ were selected.

\section{Bioinformatics Analysis}

Transcripts that were significantly up or down regulated were analyzed with DAVID [144] and Ingenuity Pathway Analysis (IPA) software ((Ingenuity Systems Inc., Redwood City, CA) and STRING [59]. DAVID generates a group of gene clusters, from a supplied gene list, using an algorithm such that the genes in a cluster have similar biological meaning or function. Due to few expression changes observed with LDL treatment, we focused on analyzing the biological processes that were altered by the oxLDL treatment by choosing "GOTERM_BP_ALL" as a data source. The functional 
annotation clusters use the kappa statistics (measure the degree of the common genes between two annotations), which give clusters of annotated terms that share common genes by using medium classification stringency. Annotation clusters were pooled from both the upregulated and downregulated list and only enriched terms with FDR $<20 \%$ were interpreted with reflected biological processes.

\section{qPCR Analysis}

cDNA was prepared from the isolated mRNA $(1 \mu \mathrm{g})$ by reverse transcription using random hexamers and Maxima $\mathrm{H}$ Minus reverse transcriptase according to the manufacturer's recommendations (Thermo Fisher Scientific ). Real time qPCR analyses were performed using gene specific primers and Power SYBR ${ }^{\mathrm{TM}}$ Green PCR Master Mix (4367659) with a StepOnePlus SYBR green system (Applied Biosystems).Primers used are: HMOX1, AGA CTG CGT TCC TGC TCA ACA T(forward), GGG GCA GAA TCT TGC ACT TTG T(reverse); GCLM, CAT TTA CAG CCT TAC TGG GAG G (forward), CGT GCG CTT GAA TGT CAG G (reverse); NQO1, AAA AGA AGC TGG AAG CCG CA (forward), AGG ATT TGA ATT CGG GCG TC(reverse); HMGCR, TCA AAG GGT ACA GAG AAA GCA C(forward), TAT GCT CCC AGC CAT GGC AG(reverse); ABCA1, GCA CTG AGG AAG ATG CTG AAA(forward), AGT TCC TGG AAG GTC TTG TTC AC(reverse); LDLR, CTG GAC CGG AGC GAG TAC AC (forward), TGG GTG CTG CAG ATC ATT CTC (reverse); SQLE, TGG GCA TTG CCA CTT TCA C(forward), GGC CTG AGA GAA TAT CCG AGA AG(reverse); BHLHE40, AAT CAT TGC CCT GCA GAG TG(forward), CGA AGA CTT CAG GTC CCG AG(reverse); PER1, CTG CTA CAG GCA CGT TCA AG(forward), CTC AGG GAC CAA GGC TAG TG(reverse). Briefly, the reaction mixture consisted of $10 \mathrm{ng}$ cDNA, 0.6 $\mu \mathrm{M}$ primers in a final volume of $50 \mu 1$ reaction mixture. Each cycle consisted of a denaturation step at $95{ }^{\circ} \mathrm{C}$ for 15 seconds, annealing and extension steps at $55{ }^{\circ} \mathrm{C}$ for 1 min for a total of 40 cycles. A melt curve was generated for each reaction to confirm the absence of non-specific amplification products. GAPDH was used for normalization. All experiments were performed with a minimum of 6 technical replicates and three biological replicates.

\section{Results and Discussion}

Accumulation of oxLDL is believed to contribute to the pathogenesis of AMD by establishing a low-grade, chronic inflammatory state in the macula. The molecular mechanisms that link the accumulation of oxLDL to the development of AMD are poorly understood. Recent studies in primary human fetal RPE (hf-RPE) suggest that oxLDLinduced cytotoxicity requires binding through the CD36 cellular scavenger receptor and the activation of the NLRP3 inflammasome [145]. In agreement with this and other studies, e.g. [146], we determined that CD36 is a major contributor to the cellular uptake of oxLDL in retinal pigment cells: a drastic decrease of oxLDL uptake was observed as measured by quantitative flow cytometry or confocal microscopy imaging analysis following pretreatment of cells with the CD36-specific irreversible inhibitor sulfo-N- 
succinimidyl oleate (SSO) [147] (Figure 3-2A and B). To allow for the detection of a robust and early gene expression response without significant cytotoxicity, various exposure times and oxLDL concentrations were tested. We established that $2 \mathrm{~h}$ and $4 \mathrm{~h}$ treatment of ARPE-19 cells with $100 \mu \mathrm{g} / \mathrm{ml}$ of oxLDL had no detectable cytotoxicity as measured by the LDH cytotoxicity assay (Figure 4-1). Cell treatment with $100 \mu \mathrm{g} / \mathrm{ml}$ of oxLDL for 2 and $4 \mathrm{~h}$ triggered a reproducible, robust gene expression response as shown by Pearson's Correlation in Figure 4-2.

To determine the specificity of the oxLDL-triggered gene expression changes in the cells, they were also treated for $4 \mathrm{~h}$ with $100 \mu \mathrm{g} / \mathrm{ml}$ of LDL, the non-oxidized form of oxLDL. The microarray data showed a marked difference in gene expression profiles between the LDL and oxLDL treatment. The 4h oxLDL treatment caused the highest number of differentially expressed genes: 727 genes were differentially expressed as compared to controls, whereas the LDL treatment caused expression changes in 241 genes. Only 32 genes were commonly up- or down-regulated between the two treatment groups (FDR $<5 \%$, fold change $\geq 1.5$ ) (Figure 4-3A). The oxLDL-induced change in gene expression was time dependent, with an increase in the number of differentially expressed transcripts at $4 \mathrm{~h}$ (Figure 4-3B). Interestingly, the data showed that in response to $4 \mathrm{~h}$ oxLDL treatment the largest number of differentially expressed genes were downregulated (Figure 4-3C), even though the transcripts with the largest changes in expression were up-regulated genes (Figure 4-4A).

A heat map of the differentially expressed genes (FDR $<5 \%$ and fold change $\geq$ 2.0) provides an overall view of the effects of the different treatments: (i) oxLDL triggered a much stronger transcriptomics response than LDL; (ii) the oxLDL effects were time-dependent and much more pronounced after $4 \mathrm{~h}$ treatment (Figure 4-4B). Analysis of the $4 \mathrm{~h}$ oxLDL-induced DEGs (FDR $<5 \%$, fold change $\geq 2.0$ ) by DAVID functional annotation tool identified 53 annotation clusters for the up regulated gene transcripts and 73 for the down regulated ones, respectively. Selected enriched annotation clusters with statistically significant annotation terms (Benjamini $<0.005)$ with associated biological processes are shown in Figure 4-5.

The analysis of the up-regulated gene transcripts identified functional annotation term clusters that contain elements relevant to anti-oxidative stress response, among others, while analysis of the down-regulated transcripts identified functional annotation term clusters including ones that contain elements that are relevant to lipid biosynthesis and circadian rhythm regulation. Gene expression changes of selected elements from the identified functional annotation term clusters from DAVID analysis at $4 \mathrm{~h}$ oxLDL (FDR $<5 \%$, fold change $\geq 2.0$ ), and the corresponding changes observed at $2 \mathrm{~h}$ oxLDL are shown in Table 4-1. Gene expression results observed for the $4 \mathrm{~h}$ LDL treatment (FDR < $5 \%$, fold change $\geq 2.0$ ) are summarized in Table 4-2. Taken together, the data shown in Table 4-1 and 4-2 demonstrate the time-dependency and selectivity of the gene expression response.

To validate the microarray results, selected transcripts were quantified by qPCR. The validated transcripts belong to three different gene functional pathways - anti- 


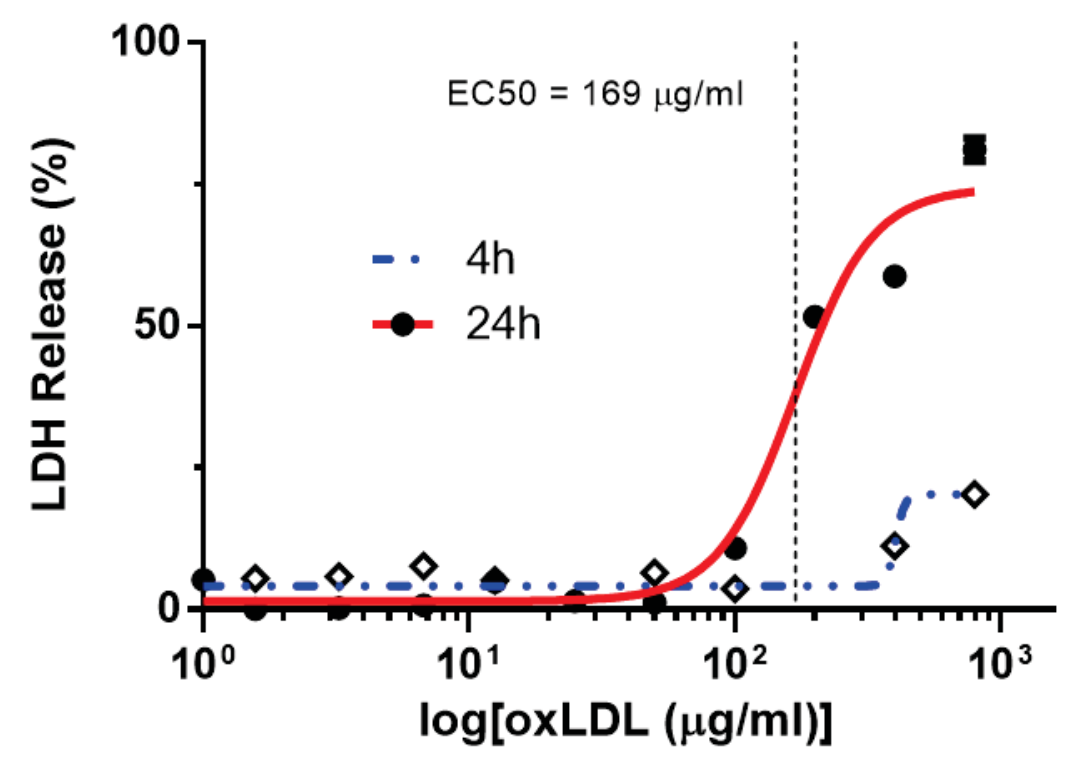

Figure 4-1. Cytoxicity of OxLDL in ARPE-19 cells.

ARPE-19 cells were treated with 10 different concentration of oxLDL for $4 \mathrm{~h}$ and $24 \mathrm{~h}$. After treatment, the medium from each well was collected, and cytotoxicity was measured by LDH Assay according to the manufacturer instruction. 

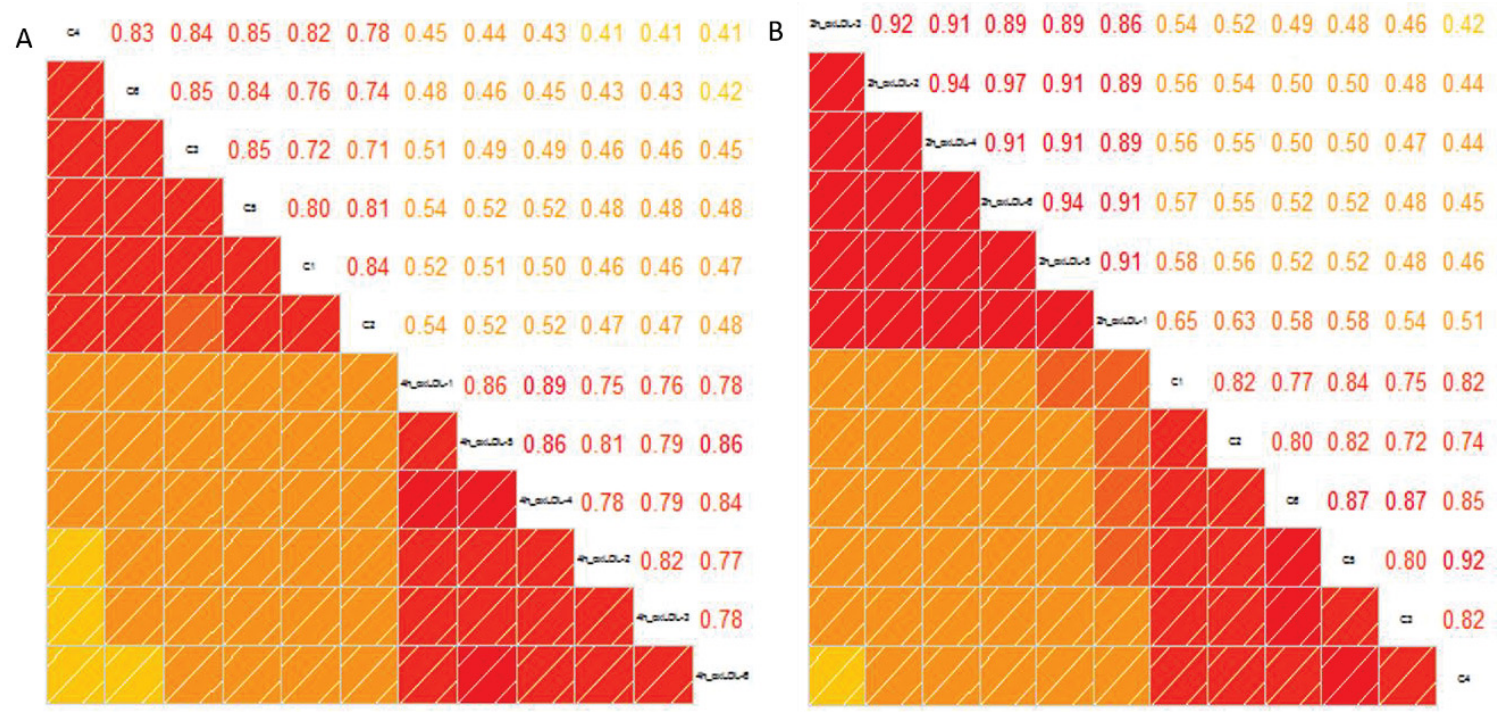

Figure 4-2. OxLDL for 2 and $4 \mathrm{~h}$ triggered a reproducible, robust gene expression response.

Pearson's correlation between (A) 4h oxLDL and control (B) 2h oxLDL and control 
A

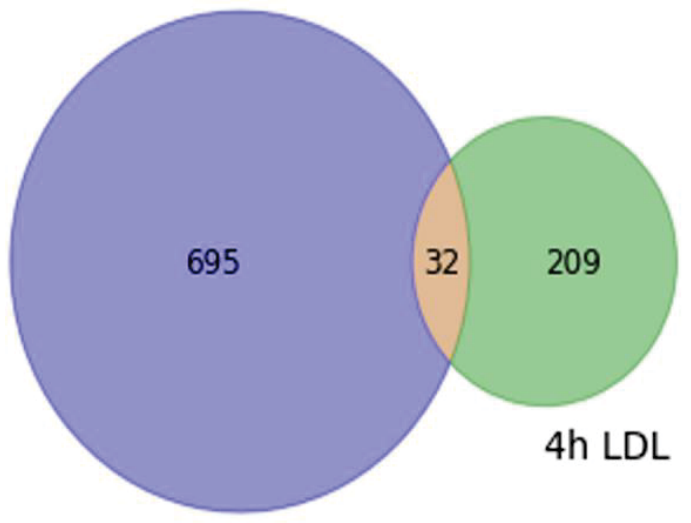

4h oxLDL
B

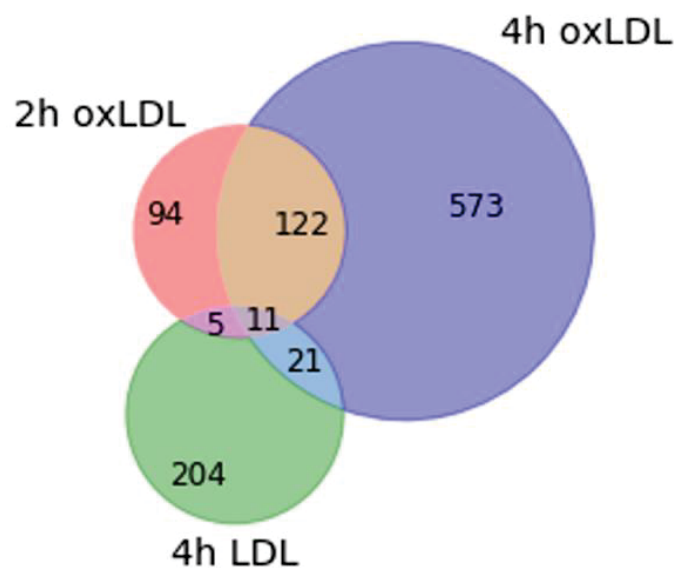

C

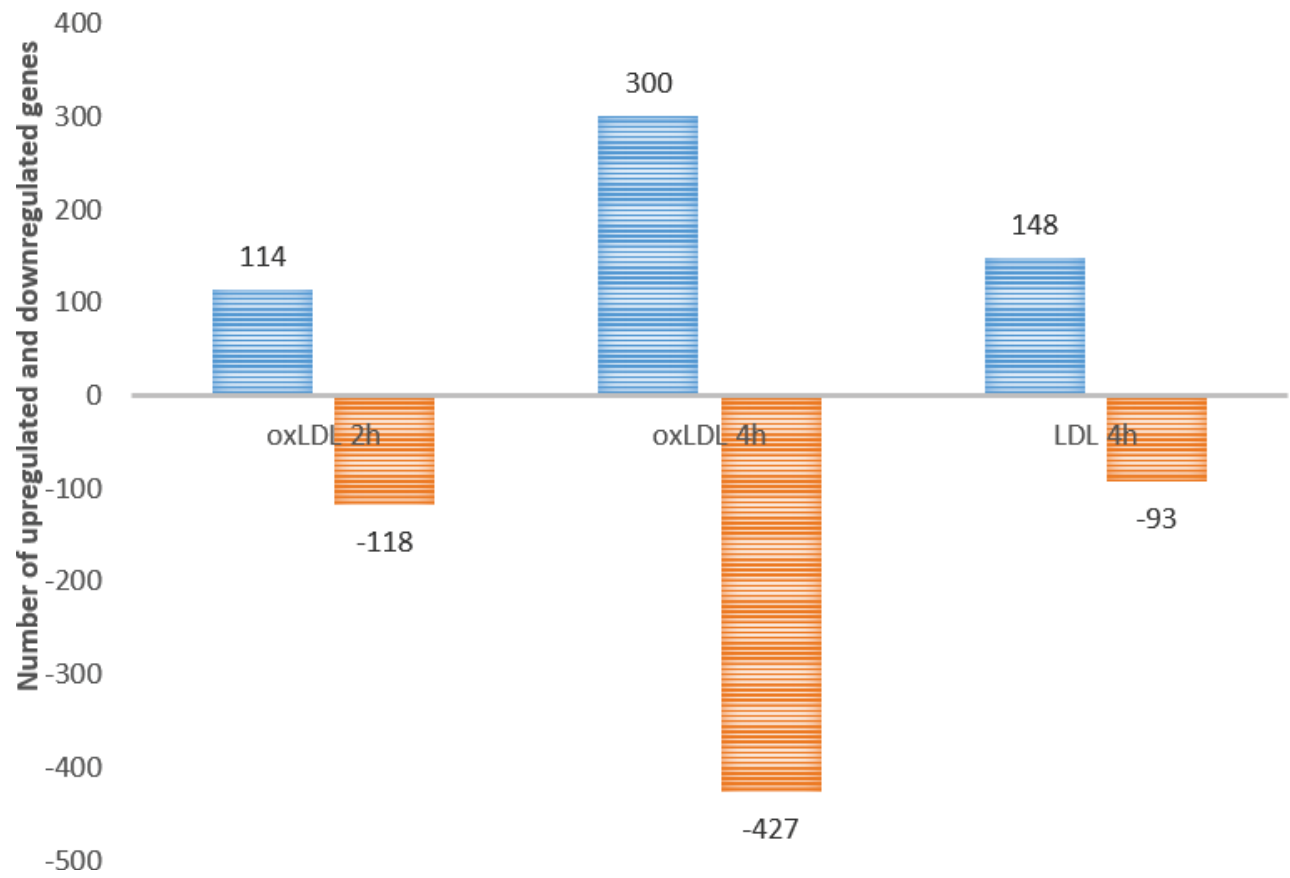

Figure 4-3. Gene expression changes caused by oxLDL and LDL treatment. (A): Venn diagrams illustrating the common and unique genes expressed by LDL and oxLDL treatment at $4 \mathrm{~h}$ and (B) $2 \mathrm{~h}$ and $4 \mathrm{~h}$ (fold change $>1.5$, FDR $<5 \%$ ). (C). Bar graph showing the number of up- and down-regulated genes due to each treatment at $2 \mathrm{~h}$ and $4 \mathrm{~h}$. 
A

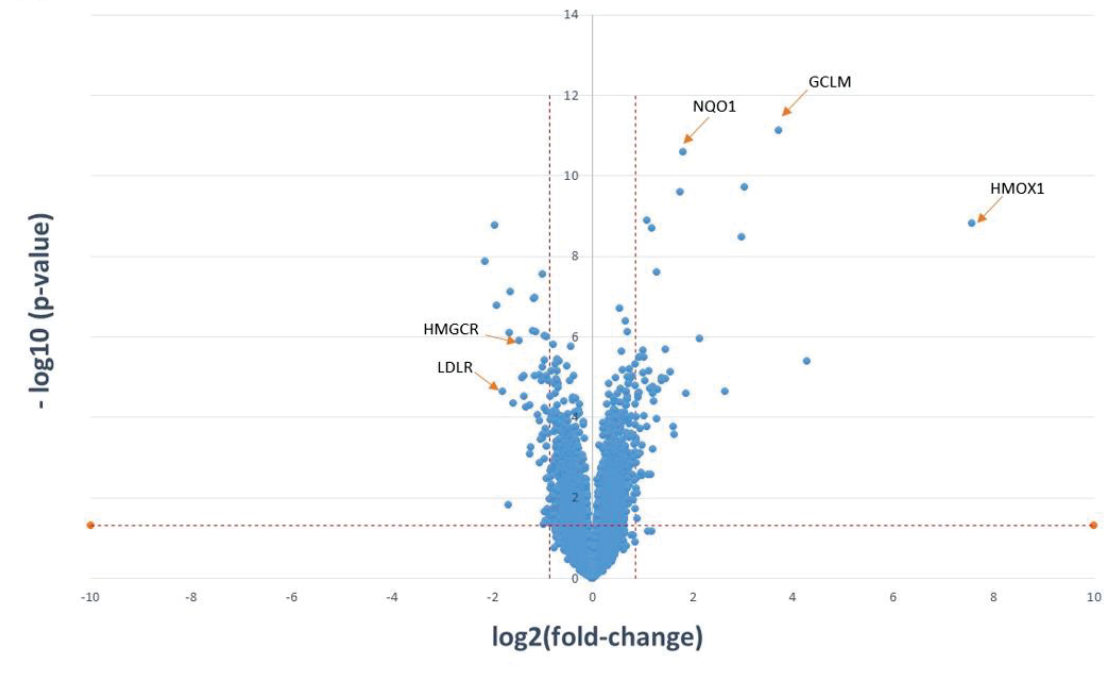

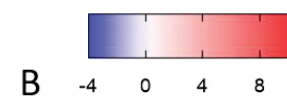

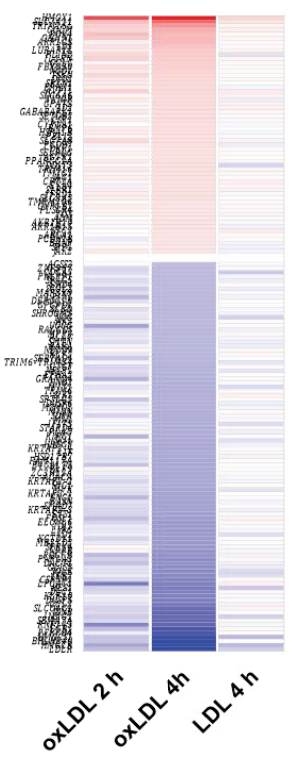

Figure 4-4. Volcano plot and heat map for mRNA expression.

(A) Scatter-plot of the mean expression fold-changes versus significance. Left, downregulated; right, up-regulated genes. The red dashed lines represent the threshold limits for statistical significance $(p=0.05)$. (B) Heat map for a set of genes with fold changes $\geq$ or $\leq 2(\mathrm{FDR}<5 \%)$ at $2 \mathrm{~h}$ and $4 \mathrm{~h}$ oxLDL treatment and $4 \mathrm{~h}$ LDL treatment. 

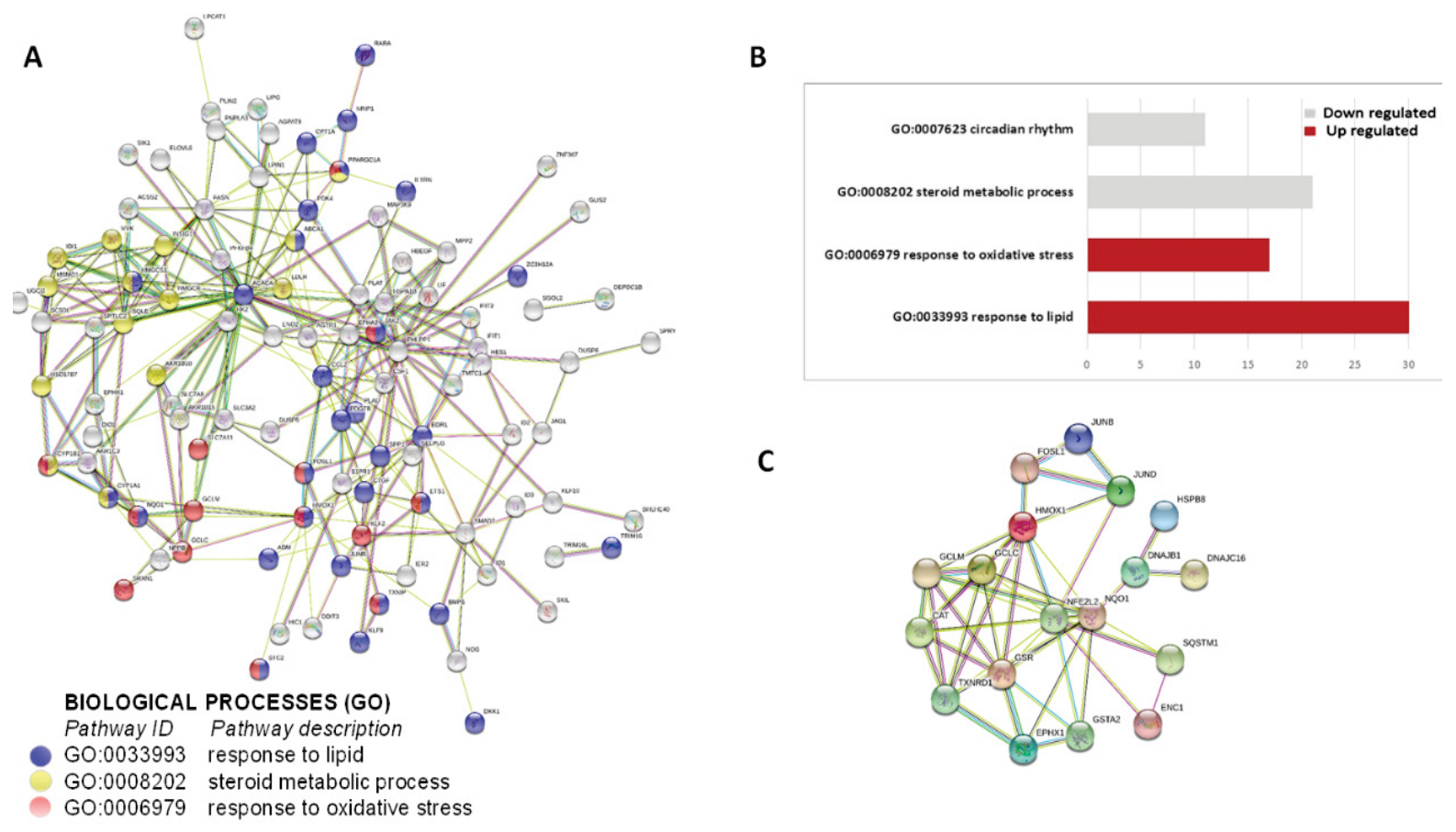

Figure 4-5. Molecular networks and pathways.

(A) STRING gene interaction network. Selected significantly enriched biological processes are highlighted with colored circles. (B) Selected functional clustering results by DAVID. Gray bars: biological processes down-regulated by oxLDL; red bars: processes up-regulated by oxLDL. (C) STRING gene interaction network encompassing members of Nrf2-controlled oxidative stress response. Bioinformatics analyses were carried out for a set of differentially expressed genes with more than 1.8 fold change (FDR $<5 \%$ ) for 4 h oxLDL treatment.. 
Table 4-1. Gene expression changes due to oxLDL at $2 \mathrm{~h}$ and $4 \mathrm{~h}$.

\begin{tabular}{lrc}
\hline Pathways/Genes & 2 h OxLDL/Con & 4 h OxLDL/Con \\
\hline Response to oxidative stress & 129.6 & \\
HMOX1 & 6.1 & 624.7 \\
SLC7A11 & 3.1 & 52.0 \\
GCLM & 1.2 & 9.6 \\
GCLC & 1.7 & 3.5 \\
NQO1 & 1.7 & 3.4 \\
SRXN1 & 1.8 & 2.8 \\
TXNIP & 1.6 & 2.7 \\
HSPA1B & 1.6 & 2.3 \\
CYP1B1 & -1.0 & 2.1 \\
CYP1A1 & & 2.4 \\
Lipid metabolism & 1.1 & \\
ABCA1 & -1.0 & 2.0 \\
ACSS2 & -1.4 & -2.0 \\
SC5D & -1.2 & -2.0 \\
MSMO1 & -1.9 & -2.3 \\
SPTLC2 & -1.4 & -2.3 \\
HSD17B7 & -1.3 & -2.5 \\
ACACA & -1.3 & -2.5 \\
MVK & -1.4 & -2.6 \\
FASN & -1.3 & -2.7 \\
IDI1 & -1.4 & -2.8 \\
SQLE & -1.7 & -3.2 \\
INSIG1 & -1.8 & -4.7 \\
HMGCS1 & -2.6 & -10.2 \\
HMGCR & -2.0 & -12.6 \\
LDLR & & -12.7 \\
Circadian rhythm & 2.2 & \\
ID3 & 1.4 & 5.2 \\
ID2 & 1.6 & 2.5 \\
PPARGC1A & -1.2 & -2.2 \\
PHLPP1 & -1.4 & -2.2 \\
NRIP1 & -0.7 & -2.5 \\
KLF9 & -1.6 & -2.7 \\
ETS1 & -1.2 & -2.8 \\
ID1 & -1.1 & -3.7 \\
KLF10 & -1.9 & -7.9 \\
EGR1 & 1.0 & -1.8 \\
PER1 & -1.8 & \\
BHLHE40 & & \\
HSL & \\
\hline
\end{tabular}


Table 4-2. Gene expression changes due to LDL at $4 \mathrm{~h}$.

\begin{tabular}{lc}
\hline \multicolumn{1}{c}{ Pathways / Genes } & $\mathbf{4}$ h LDL/Con \\
\hline Response to oxidative stress & \\
HMOX1 & 3.6 \\
Lipid metabolism & \\
FAXDC2 & 2.1 \\
HMGCS1 & -2.0 \\
HMGCR & -2.1 \\
Other & \\
PORCN & 2.3 \\
EHD3 & 2.0 \\
SIGMAR1 & 2.0 \\
CDC42 & -2.1 \\
NUDT6 & -2.2 \\
PPM1B & -2.6 \\
HIST1H2BM & -3.6 \\
\hline
\end{tabular}


oxidative stress response, lipid metabolism and circadian rhythm processes. The qPCR results were consistent with the microarray data Table 4-3.

\section{OxLDL and Anti-Oxidative Stress and Inflammatory Response}

Ingenuity pathway analysis (IPA) of this group of up regulated genes identified the nuclear factor erythroid 2-related factor 2 (NRF2) transcription factor as the main regulatory element of the antioxidant response. Age-related macular degeneration (AMD) manifests with the formation of drusen, originating from cellular and inflammatory debris that accumulates between the retinal pigment epithelium (RPE) and the Bruch's membrane. Impairment of the innate immune response, high fat/cholesterol diet and oxidative stress play a role in the onset and development of AMD. The retina is very vulnerable to oxidative stress: it has a high metabolic rate, high oxygen tension, high content of polyunsaturated fatty acids (PUFAs), the presence of retinal pigments and has exposure to light. All these factors contribute to the presence of a highly oxidative cellular and tissue milieu in which DNA, lipids, and proteins become oxidized. The molecular and cellular mechanisms that lead from oxidative stress to cell dysfunction, cell death, and tissue damage are poorly understood. To date, the strongest epidemiological evidence indicates two main risk factors for AMD: (i) genetic polymorphism in genes involved in complement regulation and (ii) environmental factors, i.e., cigarette smoking. Oxidative stress, specifically protein oxidation and its deleterious consequences on cell viability, is sufficient to mechanistically account for both observations. Weismann et al. demonstrated this clearly by showing a mechanistic link between $\mathrm{CFH}$ polymorphism, the highest genetic risk for developing AMD, and oxidative stress-induced protein modification [148]. To counter the damaging effects of protein oxidation, the RPE cells have developed numerous anti-oxidant protective mechanisms. One central component of the protective network is nuclear factor erythroid-2 related factor 2 (NRF2), which in the presence of intracellular reactive oxygen species (ROS), induces the expression of various detoxifying enzymes including enzymes of the glutathione redox system, catalases (CAT), superoxide dismutases (SOD), and the aldehyde dehydrogenases (ALDH). The NRF2-mediated transcription regulation is a well characterized anti-oxidative response mechanism (Batliwala, Xavier et al. 2017), which involves, the release of the inactive Nrf2 protein from its interaction with Kelch-like ECH-associated protein 1 (Keap1), translocation to the nucleus and dimerization with Maf proteins, binding to the antioxidant response element (ARE) located in the promoter region of target genes and initiation of transcription.

One of the target genes known to be up-regulated by the activation of Nrf2 during the acute phase of an anti-oxidative response is HMOX1. Kronke et al., have shown that the increase of protein levels of heme oxygenase 1 (HO-1) in human umbilical vein endothelial cells following treatment with oxidized 1-palmitoyl-2-arachidonoyl-snglycero-3-phosphorylcholine (OxPAPC), a component of oxLDL, in addition to NRF2 transcription activation, involves the activation of the cAMP-responsive element-binding protein (CREB) through a signaling cascade that includes the mitogen-activated protein 
Table 4-3. Validation of gene expression changes by qPCR.

\begin{tabular}{cccc}
\hline Gene & 2 h OxLDL/Con & 4 h OxLDL/Con & 4 h LDL/Con \\
\hline HMOX1 & $34.3^{* * *}$ & $175.7^{* * *}$ & $11.9^{* * *}$ \\
GCLM & $4.4^{* * *}$ & $12.6^{* * *}$ & $1.8^{* *}$ \\
NQO1 & $2.0^{* * *}$ & $6.8^{* * *}$ & 1.1 \\
HMGCR & $-2.3^{* *}$ & $-7.9^{* * *}$ & $-5.1 * * *$ \\
ABCA1 & $1.3^{* * *}$ & $2.1 * * *$ & $1.9^{* * *}$ \\
LDLR & $-1.2^{*}$ & $-4.7^{* * *}$ & $-5.4^{* * *}$ \\
SQLE & $-1.2^{* * *}$ & $-2.3^{* * *}$ & $-2.1 * * *$ \\
BHLHE40 & $-2.1 *$ & $-15.9^{* * *}$ & $1.0^{*}$ \\
PER1 & $-1.1 * * *$ & $-3.1 * * *$ & 1.2 \\
\hline
\end{tabular}

Note: ${ }^{*} \mathrm{p}<0.05 ; * *<0.01 ; * * * \mathrm{p}<0.001$ ( $\mathrm{n}=6$ biological replicates $)$ 
kinase p38 and ERK [149]. These findings, suggest that cellular anti-oxidative stress responses to oxidized phospholipids require the activation of multiple signaling cascade pathways that lead to a strong up-regulation of $\mathrm{HO}-1$.

In agreement with above mentioned studies, Ingenuity Pathway Analysis (IPA) of our transcriptomics data clustered several of the most up-regulated genes around the NRF2 transcription factor (Figure 4-5C). Within this cluster of up-regulated transcripts, $H M O X 1$ had the highest up-regulation at 2 and $4 \mathrm{~h}$ oxLDL treatment $(>129$ and 600 -fold increase, respectively) in entire data set. HO-1 catalyzes the breakdown of heme into biliverdin, iron and carbon monoxide [150]. It is up-regulated in chronic and acute inflammation $[151,152]$ and it has anti-oxidative stress and anti-inflammatory properties $[153,154]$. Interestingly, polymorphism of the $H M O X 1$ gene, which results in an aspartic acid to histidine substitution at position 7 in the protein, has been shown to be associated with increased susceptibility to dry AMD [155]. In addition to HMOX1, treatment of ARPE-19 cells with oxLDL also induced a strong NRF2-dependent up-regulation of other gene transcripts, whose translation products have anti-oxidative enzymatic activity including: GCLM (Glutamate-Cysteine Ligase Modifier Subunit), NQO1 (NAD(P)H dehydrogenase [quinone] 1), GSR (Glutathione-Disulfide Reductase), GCLC (GlutamateCysteine Ligase Catalytic Subunit), CAT (Catalase) and FTL (Ferritin Light Chain).

Studies have shown the essential role of immunity or inflammatory factors involved in the development and progression of AMD $[1,156,157]$. oxLDL is known to induce inflammatory responses in various tissue types [158-160]. Moreover, one of the most cytotoxic components of oxLDL, 7-ketocholesterol, is known to activate inflammatory responses in the ARPE-19 cells [161-163]. Ingenuity pathway analysis (IPA) of our transcriptomics data identified a set of genes involved in inflammatory responses that were up-regulated following the $2 \mathrm{~h}$ treatment with oxLDL including CEBPB (CCAAT/Enhancer Binding Protein Beta), IL1RN (Interleukin 1 Receptor Antagonist), JAK2 (Janus kinase 2), CXCL8 (Interleukin 8), IL6R (Interleukin 6 receptor) and IL1RL1 (Interleukin 1 Receptor Like 1). CEBPB is a well characterized transcription factor that regulates the expression of genes involved in immune responses [164]. Similar to our study, CEBPB has been shown to be up-regulated in RPE cells following the treatment with agents known to cause oxidative damage - homocysteine [165] and $\mathrm{N}-(4-h y d r o x y p h e n y l)$ retinamide (4HPR) a retinoic acid derivative that causes oxidative damage through the generation of reactive oxygen species (ROS) [166].

\section{OxLDL and Circadian Rhythms}

The photoreceptors shed their outer (POS) segment tips daily through cellular processes dependent on light conditions [167] and the RPE play a critical role in removing the aged POS tips through phagocytosis, a process synchronized with the POS shedding and controlled by circadian rhythm mechanisms $[168,169]$. In fact, there is a strong link between ocular physiology and circadian rhythm with the circadian rhythmicity of the RPE playing a critical role for the support of the photoreceptors and retinal function [170] in all vertebrate animals, including humans [171]. Phagocytosis in 
RPE is known to increase ROS production, which can alter the renewal process such as delayed termination of shedding or defect in RPE digestion and can cause accumulation of lipofuscin [172]. The accumulation of lipofuscin is one of the major risk factors associated with macular degeneration $[173,174]$. However, the mechanistic links between phagocytosis of POS, accumulation of ROS and lipofuscin, dysfunction of the circadian rhythm and AMD onset and development are poorly understood [175]. Various studies have shown that disruption of circadian rhythm promotes inflammation in mice as well as in humans [176-178], and disruption of circadian rhythmicity in the retina results in increased retinal inflammation in a diabetic mouse model [179]. Moreover, mouse treated with dexamethasone and triamcinolone acetonide, powerful anti-inflammatory agents, caused up-regulation of genes involved in circadian rhythm regulation one week after treatment [180]. Since oxLDL is a well-known pro-inflammatory agent, its effects on circadian rhythm genes may be consequent to an early inflammatory response.

A novel gene pathway affected by oxLDL-induced oxidative stress was uncovered by our study and relates to the regulation of gene transcripts known to participate in circadian rhythm mechanisms Table 4-1. All circadian rhythm-associated transcripts were down regulated, and the down regulation was observed only in the $4 \mathrm{~h}$ treatment group suggesting a time delay between treatment and response. The most down regulated gene was the class E basic helix-loop-helix protein 40 (BHLHE40), a transcriptional repressor involved in the regulation of the circadian rhythm. Other down regulated genes were kruppel-like factor 9 and 10 (KLF9 and KLF10). KLF9 is a circadian transcription factor that regulates cell proliferation. KLF10 is a transcriptional repressor that binds to the GC box sequence in the promoter sequence of the ARNTL (BMAL1) clock component and represses its transcriptional activity. Also, as a result of the oxLDL $4 \mathrm{~h}$ treatment, we observed a down regulation of the period circadian protein homolog 1 transcript (PER1), a core component of the circadian clock. The mammalian cellular circadian molecular system consists of core clock genes Period (PER) 1 and PER 2, cryptochrome (CRY) 1 and CRY 2, CLOCK, and Arntl or BMAL1 ( Aryl hydrocarbon receptor nuclear translocator like) genes [181]. These genes generate circadian rhythm through coupled transcription/translocation feedback loops [180]. The CLOCK and BMAL1 form the transactivating, i.e., the positive components of the feedback loop and PER and CRY the transinhibitory, i.e., the negative components [182].

These results suggest a potential role of oxLDL in the inhibition of the RPE phagocytic and metabolic clearing processes through interference with circadian rhythm related molecular processes. How the uptake of oxLDL might interfere with circadian rhythm processes remains to be further investigated. Nevertheless, based on our data and published literature, we propose a molecular mechanism that could explain the observed down regulation of circadian rhythm transcripts by oxLDL, which involves the activation of the aryl hydrocarbon receptor (AhR) by oxidized lipid or protein components of oxLDL. Activation of AhR can induce two separate transcription pathways [183]: 1. Activated AhR binds to ARNT and induces xenobiotic metabolism through binding to dioxin response elements (DREs) and transcription of genes with detoxifying functions such as cytochrome P450, family 1, member 1A (Cyp 1A1). Our data shows up regulation of Cyp 1A1. 2. Activated AhR binds to Bmall and prevents its binding to Clock thus inhibiting the formation of the Bmal1-Clock heterodimer essential for the transcription of 
circadian genes, which are needed for phagocytosis. In support of our working hypothesis, our data show a down regulation by oxLDL of major circadian genes including Per 1, BHLHE40, and KLF10 (Figure 4-6).

\section{OxLDL and Autophagocytosis}

The $4 \mathrm{~h}$ oxLDL treatment induced the up-regulation of the autophagy inducer sequestosome1 (p62/SQSTM1). The p62 is one of the most important genes involved in regulating the packing and transporting of ubiquitinated, misfolded and aggregated proteins for clearance via autophagy in mammalian cells [184]. Under oxidative stress conditions, induction of the p62 gene is regulated by NRF2 and contributes to activation of NRF2-controlled target genes through a positive feedback loop that enhances the cellular anti-oxidative stress response that includes the degradation and removal of the NRF2 inhibitor KEAP1 protein via autophagocytic mechanisms [185]. In RPE cells, it has been shown that p62 plays a central role in improving cellular viability when the proteasomal pathways are disrupted due to oxidized protein aggregates [186]. Studies in other cell types have shown that p62/SQSTM1 positively regulates NOD2-induced overexpression of genes related to inflammation such as tumor necrosis factor alpha (TNF- $\alpha$ ) and interleukin-1 $\beta$ (IL-1 $\beta$ ) [187]. Our transcriptomics studies confirm that oxLDL induces the p62-KEAP1-NRF2 stress response axis in ARPE-19 cells [185].

\section{OxLDL and Lipid Metabolism}

The accumulation of lipids and lipid derivatives in the sub retinal space and the association of genes involving lipid metabolism such as APOE (apolipoprotein E), CETP (cholesteryl ester transfer protein), LIPC (hepatic lipase gene) and LPL (lipoprotein lipase) with AMD, provides support for the postulated role lipids play in the pathogenesis of AMD [188-191]. In our study, we found 62 genes involved in lipid metabolic pathways that were differentially expressed in the $4 \mathrm{~h}$ oxLDL treatment group.

The highly down-regulated group of transcripts included genes involved in fatty acid synthesis such as FASN (fatty acid synthase), BDH1 (3-hydroxybutyrate dehydrogenase 1), ACACA (acetyl-CoA carboxylase alpha), HSD17B7 (hydroxysteroid 17-beta dehydrogenase 7), IDI1(isopentenyl-diphosphate delta isomerase 1), the transcriptional activator required for lipid homeostasis SREBF1 (sterol regulatory element binding transcription factor 1), as well as the LDL receptor, low density lipoprotein receptor (LDLR), which had a 12 fold decrease in expression as compared to the control cells. In contrast, the lipid efflux gene ABCA1 involved in cholesterol efflux was up-regulated.

Excess intracellular cholesterol is known to suppresses the formation of new lowdensity lipoprotein receptor $(L D L R)$ and cholesterol synthesis [192]. In agreement with these findings, our data show that the treatment with oxLDL, and to a much lower level 


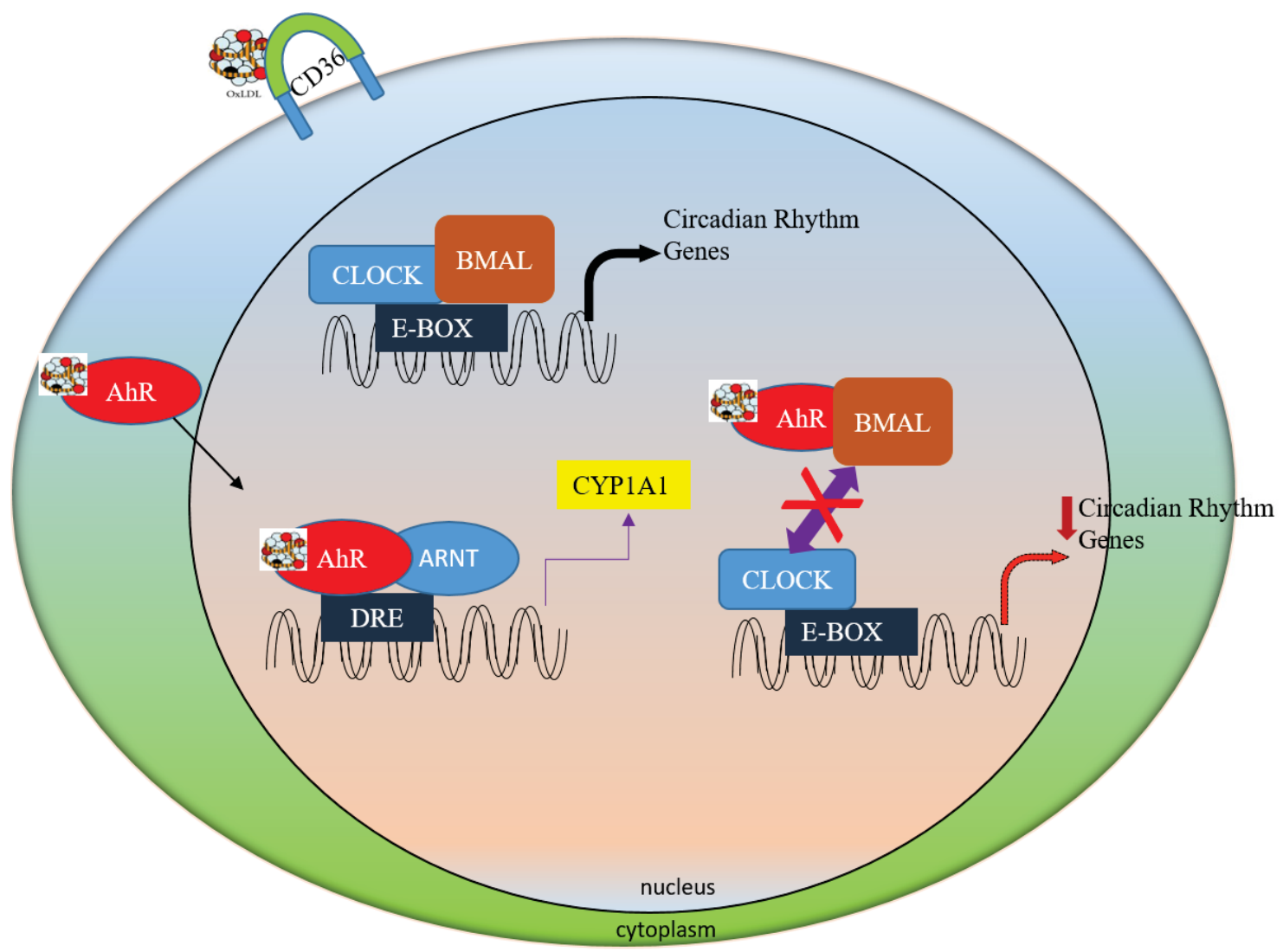

Figure 4-6. OxLDL induced molecular change in regulation of circadian rhythm. 
with LDL, had a major impact on the expression of genes whose protein products regulate lipid and steroid metabolism. Interestingly, following a $4 \mathrm{~h}$ treatment with oxLDL, the most down regulated transcript in the entire data set was $L D L R$. LDLR is a cell membrane-bound protein receptor responsible for the uptake of LDL, but not oxLDL. The down regulation of the LDLR receptor occurs in response to the intracellular release of the oxLDL lipid cargo, which include cholesterol and triglycerides. The increase of the intracellular levels of cholesterol and triglycerides triggers signaling mechanisms that result in the down regulation of gene transcripts whose protein products are enzymes involved in the uptake of extracellular LDL and the de-novo synthesis of cholesterol. In addition to strong down regulation of $L D L R$, our data shows a significant down regulation of 3-hydroxy-3-methylglutaryl-CoA (HMGCR), 3-hydroxy-3-methylglutarylCoA synthase 1 (HMGCS1) and other gene transcripts part of the cholesterol synthetic pathway. The $H M G C R$ protein product is the rate-limiting enzyme in the synthesis of cholesterol and nonsterol isoprenoids, while the HMGCS1 transcript codes for another enzyme part of the cholesterol biosynthetic pathway. Expression of $L D L R, H M G C R$, and $H M G C S 1$ is regulated by the sterol regulatory element-binding protein 1 (SREBF1) transcription factor, also significantly down regulated in the $4 \mathrm{~h}$ oxLDL treatment group. In contrast to the strong down regulation of gene transcripts whose protein products regulate cholesterol and lipids uptake and de novo synthesis, our data show significant up regulation of gene transcripts whose products are involved in cholesterol and lipids degradation and cellular efflux. In this functional group of gene transcripts up regulated by the $4 \mathrm{~h}$ oxLDL treatment was the ATP binding cassette subfamily A member 1 $(A B C A 1)$.

\section{Conclusion}

The significant differences observed in the gene transcription response to oxLDL versus LDL treatment confirm the ability of the ARPE-19 cells to recognize and react to oxidation specific epitopes (OSE) present on LDL (i.e., oxLDL). Furthermore, due to the OSE heterogeneous nature, different cellular defense mechanisms are activated, some that have the Nrf2 as the major transcription factor, other AhR, PPARG, etc. while the non-OSE containing LDL component of oxLDL induces the expression of genes that mediate the down regulation of gene pathways involved in lipid metabolism and lipid synthesis. These results depict a complex cellular and molecular mechanism, which involve various pathways only partially overlapping. The oxLDL treatment caused differential expression of genes involved in oxidative stress, lipid metabolism, inflammation, autophagy, and circadian rhythm; all of these pathways are known to be involved in the pathogenesis of AMD. While the earlier studies on the oxLDL induced transcriptome alteration on ARPE-19 were conducted at longer time points, our study shows the early events that occur in the ARPE-19 cells as they are exposed to oxLDL. Understanding the early molecular pathways that are regulated due to the exposure of oxLDL in the ARPE-19 cells line can provide us insight on early mechanisms going in the retinal pigment epithelium cells due to the presence of oxidized lipids in the retina. These findings then could give us targets to intervene the pathogenesis for AMD 
progression in human for development of better treatment and prevention against this multifactorial disease. 


\section{CHAPTER 5. PROTEOMIC ANALYSIS OF OXLDL TREATED ARPE-19 CELLS: A STUDY OF LC-MS/MS BASED BIOANALYTICAL PLATFORMS}

\section{Introduction}

Proteomics global-scale examination of the structure and function of proteins [14]. The study of proteins is crucial to understanding the pathological condition of various diseases as these are the key players in the metabolic pathways within the cells [193]. The importance of understanding the molecular mechanism of biological processes in human diseases has been strengthened by the establishment of biology and disease driven human proteome project (B/D-HPP) [194]. The B/D-HPP focuses on in-depth characterization and quantification of proteins by utilizing the state of art proteomics tools. This has enormously helped biological sciences and clinical community to better understand the molecular mechanism underlying various biological processes relevant to human diseases. With this regard, the human eye proteome project was started in 2013 with the goal to gather better insight on the disease mechanism in various parts of the eyes [195].

The main aims of the differential proteomic approaches are to obtain a better mechanistic understanding of biological processes in various contexts, to identify new drug targets, and to improve the diagnosis of disease via biomarker discovery [196]. The proteomic field can be divided into two main analytical flows, top-down and bottom-up, based upon the protein or peptide characterization. The top-down proteomics is the analysis of proteins by mass spectrometer without proteolytic digestion. Although the top-down proteomics has its advantages and limitations when compared to the bottom-up approach, however, the later has been increasingly adopted in proteomics. In our study, we have also applied the bottom-up approach to characterize the differential proteome. Hence, I discuss the bottom-up approach below in detail.

In the bottom-up approach, the proteins from any biological system are extracted and digested with proteolytic enzymes such as trypsin, which will yield a mixture of peptides. The peptides are then separated by chromatography and analyzed by mass spectrometer. The sample acquisition by mass spectrometer can be done by three different approaches. The first approach is the data-dependent acquisition (DDA) based approach in which first full MS scan is recorded for all the ions that co-elute at a given point in the gradient elution. Then the mass spectrometer chooses a specific number of the most abundant precursor ions after which the MS/MS scans of the fragments from the selected precursor ions are recorded. The instrument hence switches between the full scan acquisition and the fragment ion acquisition. The DDA method is one of the best established strategies in the field of bottom-up proteomics [197].

The second approach is data-independent acquisition (DIA), in which both the entire range of ions that co-elute at a given point in the gradient elution and the fragment ions (for all parent ions) are acquired. The peptide fragmentation information is then retrieved by two methods: i) by generating the pseudo fragment ion spectra that are 
constructed from the DIA data obtained, ii) by target signal extraction on the basis of previously acquired single peptide fragmentation spectra. One of the major advantages of DIA over DDA is that in DIA the entire range of possible precursor ions and their fragment ions can be analyzed unlike in DDA where only selected precursor ions are fragmented [198].

The third approach in bottom-up proteomics is the targeted proteomics. Unlike DIA and DDA, in this case, the protein of interest is already known. In targeted proteomics, only a small set of predetermined and previously identified peptides based on their $\mathrm{m} / \mathrm{z}$ and elution time are selected. These preselected specific peptides of interest are then applied to the MRM (Multiple Reaction Monitoring) or PRM (Parallel Reaction Monitoring) experiments. In MRM, a subset of the preselected ion from each MS1 scan is selected to be fragmented and measured in the MS2 scan. In PRM, however, all the ions of preselected precursor ions are measured [199].

The discovery-based proteomics utilizing mass spectrometry can be either quantitative or qualitative. The quantitative proteomics utilizes protein identification methods similar to the qualitative proteomics (as discussed in chapter 2) except that it includes an additional quantification step. The quantitative proteomics provides physiological differences between two biological samples. The quantitative analysis can be done in two ways: i) stable isotope labeling and ii) label free quantification. The majority of quantitative proteomic analysis is done using the stable isotope labeling. Various isotope labeling techniques include isotope-coded affinity tags (ICAT), isobaric tags for relative and absolute quantification (iTRAQ), isobaric labeling (tandem mass tags (TMT), SILAC (stable isotope labeling by amino acid in cell culture) and ${ }^{18} \mathrm{O}[200]$. It labels the protein either during the cell growth or during sample preparation. The labeling changes the molecular mass but does not change the chromatographic and mass spectrometric behavior. The difference in peak area between the labeled and unlabeled peptides then is used to determine the quantitative difference.

Isotope labeling is a complex process that is time consuming and expensive [201]. In recent years, label free approaches are constantly being developed. Among the numerous label free methods, quantitation based on peptide spectral counting [202] and peptide ion peak area [203] are extensively applied. In the spectral counting label-free quantification approach, peptides are quantified based upon $\mathrm{m} / \mathrm{z}$, peak area, and retention time for each ion in all experiments. In label free quantification based upon peptide ion peak area, peak intensities of MS signals are integrated across retention time at a defined mass window known as the area under the curve (AUC) or extracted ion chromatography (XIC) [204].

In this project, I applied a state-of-the-art mass spectrometer from Waters "The Synapt G2 Si". We planned to use the SDS-PAGE based platform for analyzing the differentially expressed proteome for this study as described in chapter 2 . Since, in this study we wanted to analyze the differentially expressed (i.e quantitative analysis) proteins in oxLDL treated cells the sample throughput was greater than the earlier study in which we only performed qualitative analysis. However, our lab acquired a new high resolution 
mass spectrometer within the span of four years which is better than the low resolution mass spectrometer (LTQ) that we used earlier. Waters Q-TOF is a hybrid analyzer which gives outstanding peak definition and accurate mass measurement of peptides as it provides highest dynamic mass resolution. Together with UPLC and MS ${ }^{\mathrm{E}}$, the Synapt G2 $\mathrm{Si}$ acquires data which contains a complete time resolved record of all detectable precursor and product ions. Nano UPLC enables the nano scale separation of complex tryptic digest with maximum resolution, speed, and sensitivity. UPLC utilizes sub $2 \mu \mathrm{m}$ chromatographic particle which can operate in 6000-15000 psi. The instrument uses the extra dimension of ion mobility separation that maximizes separation, selectivity, and confidence. Triwave high efficiency ion mobility separates ions on the basis of size, shape, and charge before transferring to Q-TOF for high resolution mass analysis. In QTOF mass analyzers the quadrupole is operated as ion guide in MS mode and mass selection device in MS/MS mode. The ions are then accelerated by an electric field and $\mathrm{m} / \mathrm{z}$ is determined by TOF [205]. Moreover, the additional dimension of ion mobility separates ion with the same mass providing greater analytical peak capacity. High chromatographic resolution is essential for achieving high coverage and mass spectral data quality (Figure 5-1). Data independent $\mathrm{MS}^{\mathrm{E}}$ is a revolutionary technique for data acquisition. It acquires all possible analytes simultaneously between high and low collision energy during a single chromatographic run [201].

As mentioned in Chapter 3, Age related Macular Degeneration (AMD) is one of the leading causes of vision loss affecting 30 million individual worldwide that includes 10 million elderly Americans [1]. With no FDA approved drugs available for the treatment of dry AMD and no adequate treatment options available for wet AMD, there is a dire need to better understanding the disease mechanism to intervene the disease progression. This has directed the scientific community towards the study of the disease progression of dry AMD at the molecular level. Various studies have also shown that oxidation of the lipids and lipoproteins contributes to the RPE death, suggesting the role of oxidative stress in the pathogenesis of AMD [126, 206, 207]. To this end, we used the oxidized lipoprotein (oxLDL) in the human ARPE-19 cells and accessed the differential proteome alteration due to its treatment to better interrogate the proteins alterations and interactions for understanding the molecular mechanism of AMD.

Differential proteomic analysis of ARPE-19 cells treated with other oxidative stress causing agents such as cigarette smoke extract (CSE), advanced glycation end products (AGEs) and photocoagulation has been studied earlier. A study involving the treatment of ARPE-19 cells with AGEs utilized 2D gel electrophoresis for protein separation, gel image analysis, and LC-MS/MS to identify the differentially expressed proteins [208]. Another study utilized label free quantification with LC-MS/MS to identify differentially expressed proteins due to CSE treatment [209]. Recently, Agardn et al. studied differential proteomic analysis on ARPE-19 cells due to photocoagulation [cite]. They utilized IEF for separation, iTRAQ for labelling and LC-MS/MS for identifying and quantifying changes in protein expression due to treatment [210]. 


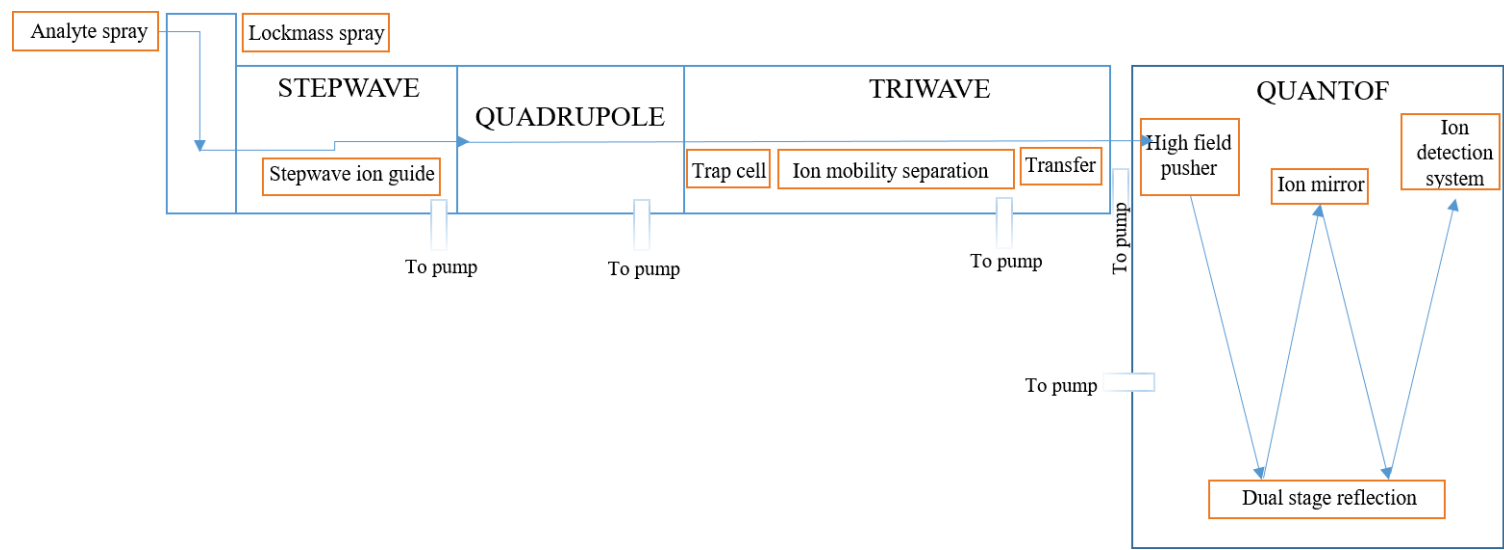

Figure 5-1. Schematic representation of SYNAPT G2-Si mass spectrometry system. 
We described early transcriptome alteration due to oxLDL treatment in the previous chapter. However, no proteomic study has been done in the context of oxLDL, which is one of the components of drusen. It would be interesting to interrogate the proteome alteration and compare it to the transcriptome alterations due to oxLDL treatment. Therefore, in this study, we interrogated the proteome alteration in ARPE-19 cells due to oxLDL treatment. We used a bioanalytical platform that uses a highresolution mass spectrometer in a data independent setting to analyze the peptides obtained from treated and untreated cells. The platform incorporated a nano UPLC for the separation of peptides in combination with LC-MS/MS.

\section{Materials and Methods}

\section{Cell Culture}

The immortalized human retinal pigment epithelium cells ARPE-19 were purchased from ATCC (Manassas, USA) and maintained in DMEM-F12 medium (ATCC, Manassas, USA) containing $2 \mathrm{mM}$ L-glutamine supplemented with $10 \%$ fetal bovine serum (ATCC, Manassas, USA) and $100 \mu \mathrm{g} / \mathrm{ml}$ (Invitrogen, Carlsbad, USA) in a humidified atmosphere with $5 \% \mathrm{CO} 2$ at $37^{\circ} \mathrm{C}$.

\section{OxLDL Treatment and Sample Collection}

Cells $(150,000)$ were seeded in 6 well plates and grown until confluent. Serumstarved $(24 \mathrm{~h})$ ARPE-19 cells were treated with $100 \mu \mathrm{g} / \mathrm{ml}$ of oxLDL and $100 \mu \mathrm{g} / \mathrm{ml}$ of LDL for $4 \mathrm{~h}$. All experiments were performed in multiple biological replicates $(n=5)$. After $4 \mathrm{~h}$ of treatment, the cells were washed with PBS, collected and stored in liquid nitrogen until further processing.

\section{Sample Preparation for Mass Spectrometry}

The samples were proteolyzed with trypsin and Lys-C (iST 8x kit, Preomics, Germany) using a sample preparation protocol provided by the manufacturer [211]. Briefly, the cell samples were lysed using lysis buffer, which denatures, reduces and alkylates proteins, for $10 \mathrm{~min}$ at $95{ }^{\circ} \mathrm{C}$. Then the samples were digested using digest buffer that had trypsin and Lyc-C mixture at $37^{\circ} \mathrm{C}$ for $3 \mathrm{~h}$. The digestion was stopped with stop buffer and then the samples were washed with wash solutions to remove hydrophobic and hydrophilic contaminants. The peptides were then finally eluted from cartridge with elution solution. Finally, the peptides were re-suspended on the LC-Load solution after completely drying with the speed vac. 


\section{LC-MS/MS Analysis}

Samples were analyzed by a Waters Synapt G2Si mass spectrometer system with a nanospray ion source interfaced to a Waters Acquity M-class UPLC system (C18 reversed-phase capillary column). Each sample was spiked with internal standards to determine the amount of protein on the column. All peptide separations were performed on a nanoUPLC liquid chromatography system (Waters Acquity M-class UPLC system). Mobile phase A was $\mathrm{H}_{2} \mathrm{O}$ containing $0.1 \%$ formic acid; mobile phase $\mathrm{B}$ was ACN containing $0.1 \%$ formic acid. Peptide samples were injected in a volume of $2 \mu 1$ using partial loop injection, onto a Symmetry C18, $5 \mu \mathrm{m}, 180 \mu \mathrm{m}$ x $20 \mathrm{~mm}$ trap column (Waters). After pre-concentration, the peptides were eluted from the trap column to the analytical column, HSS T3 C18, $1.8 \mu \mathrm{m}, 75 \mu \mathrm{m}$ x $250 \mathrm{~mm}$ (Waters), and separated with a gradient of $2-40 \%$ mobile phase $\mathrm{B}$ over $180 \mathrm{~min}$ at a flow rate of $0.3 \mu \mathrm{l} / \mathrm{min}$. The column temperature was maintained at $45^{\circ} \mathrm{C}$. The eluted peptides were analyzed on-line on a Synapt G2-Si (Waters Corporation) high-resolution ion mobility mass spectrometer (IMS). The lock mass peptide [Glu1]-fibrinopeptide B was delivered by infusion through the instrument fluidics system. It was used to improve the mass accuracy of the analysis. The quadrupole RF was set for efficient transmission of ions with $\mathrm{m} / \mathrm{z}$ of 300-2000. Data was acquired in a data-independent acquisition (DIA) mode with ion mobility separation and optimized transfer collision energy, ultra-definition $\mathrm{MS}^{\mathrm{E}}\left(\mathrm{UDMS}^{\mathrm{E}}\right)$ [212]. The TOF (Time-of-Flight) analyzer was operated in resolution mode (resolution $\geq 20,000$ FWHM, with the average mass error of $5 \mathrm{ppm}$ or less), a scan time of $0.6 \mathrm{~ms}, \mathrm{MS}^{\mathrm{E}}$ range $50-2000$ Da, and IMS separation.

\section{Data Analysis}

The data was imported with the file format (.raw) from Synapt G2Si Mass Spectrometer and analyzed by Progenesis QI software (http://www.nonlinear.com/progenesis/qi-for-proteomics/download/). Oxidation of methionine was included as variable modification and, carbamidomethyl modification of cysteine was specified as fixed modification. Trypsin was specified as a protease with maximum of one missed cleavage allowed. The ion matching requirement selection was 1 or more for fragment/peptides, 3 for fragment/proteins and 1 for peptides/protein. The data was also searched against decoy database, and the false discovery rate was set to $4 \%$ at the peptide level. Peptides were selected based on the significance measures, e.g., ANOVA p-value and fold change. Identified proteins were displayed according to the protein grouping method, which is based on a less stringent application of the parsimony principle. The differentially expressed proteins were determined based on fold change (FC) with cutoffs values of $|\mathrm{FC}|>1.5$ and $\mathrm{p}$ value $<0.05$.

\section{Bioinformatics Analysis}

The differentially expressed protein list was analyzed online using STRING and DAVID software [213]. After the proteins are identified by the protein search databases 
and tools, bioinformatics tool PANTHER was used to characterized identified proteins according to cellular, molecular and biological categories [56].

\section{Results and Discussion}

In this study, we performed qualitative and quantitative analyses of ARPE-19 cells. For the quantitative proteomic analysis, the cells were treated with oxLDL. OxLDL, known to exert oxidative stress, is present in drusen, the hallmark of AMD. Oxidative stress is considered as one of the major contributing factors for the pathogenesis of AMD. Therefore, the proteomic changes that occur in the ARPE-19 cells due to oxLDL are of great importance in understanding the molecular mechanism of oxLDL induced oxidative stress. To this end, we performed an in-depth differential proteomic analysis for oxLDL induced proteome alteration in human ARPE-19 cell lines. The ARPE-19 cells were grown to confluence and treated with $100 \mu \mathrm{g} / \mathrm{ml}$ of oxLDL and LDL for $4 \mathrm{~h}$. The cells were then collected, and performed single-step lysis, denaturation, alkylation and reduction with Preomics kit as manufacturer recommendations, to obtain a complex mixture of trypsin and Lys-C digested peptides. The peptide mixtures were separated with reverse phase nanoUPLC system from Waters Corporation. The separated peptides were then analyzed online on a Synapt G2-Si (Waters Corporation) highresolution ion mobility mass spectrometer (IMS). The data from the mass spectrometer was analyzed by Progenesis software to get the list of total proteins identified by this analytical platform and the list of differentially expressed proteins due to LDL and oxLDL treatments. The summary of the experimental approach to characterize the oxLDL induced proteome alteration in ARPE-19 cells is illustrated in Figure 5-2.

As we mentioned in Chapter 2, global scale examination of proteome in any biological system is extremely challenging, and no single analytical technique is capable of identifying and characterizing proteome complexity. Hence, we presented, in Chapter 2 , the evaluation of three different bioanalytical platforms in terms of extent and depth of proteome coverage. Among the platforms evaluated, we found SDS-PAGE based platform performed the best as it identified around 900 proteins with wide abundance level. In addition, the workflows utilized the old, low resolution LTQ instrument. Also the pre-fractionation step in the SDS-PAGE based platform where we analyzed 15 fractions per sample becomes time and resource consuming if we need to study differential protein expressions. As the study of differential proteomics needs analysis of multiple replicates which drastically increases the throughput.

In this study, we utilized a high resolution mass spectrometry combined with nano-UPLC in a data independent setting. This platform provided a better proteome coverage which identified around 2500 proteins, i.e., around 3-fold more proteins than the SDS-PAGE based analytical platform. Even though this new analytical platform does not include any pre-fractionation step, the use of nano-UPLC, state-of-the-art mass spectrometer with ion mobility, and data independent acquisition provided us better separation and better results in terms of proteome coverage. The use of nano-UPLC 


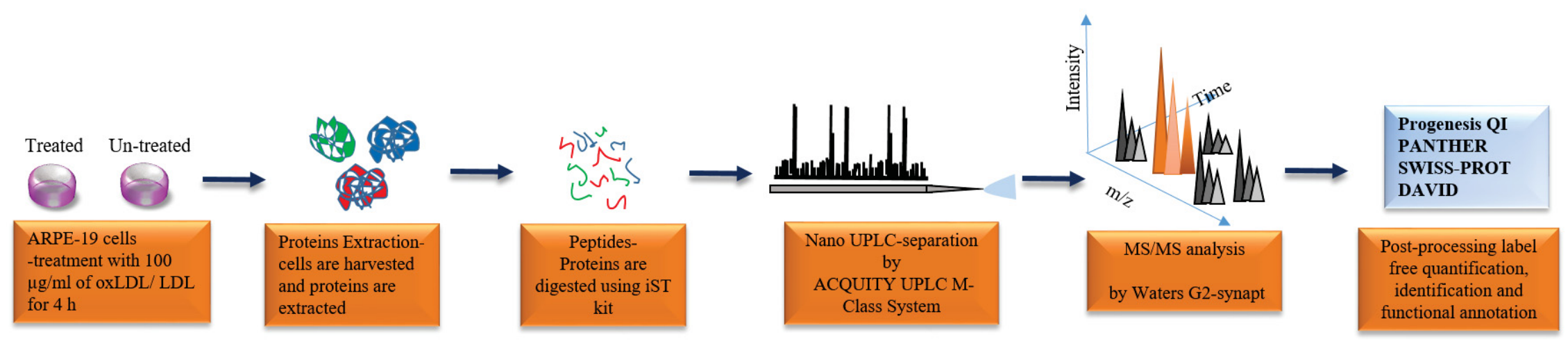

Figure 5-2. Schematic representation of bioanalytical workflow for proteomic analysis of oxLDL/LDL treated ARPE-19 cells. 
provides nano scale separation with high resolution whereas the extra dimension of ion mobility separation maximizes separation and selectivity. Moreover, the data independent $\mathrm{MS}^{\mathrm{E}}$ acquisition provides the ability to analyze all possible analytes and label free quantification of differentially expressed proteins. These combinations make the new platform robust and reproducible for unbiased discovery-based as well as for the differential proteomics.

\section{Protein Identification and Expression Profile}

RPE is a monolayer of polarized cells which is an integral part of blood retinal barrier. They play an important role in maintaining retinal hemostasis and in survival of photoreceptors. Proteomic studies of RPE would provide a great insight in better understanding its function in diseased and healthy conditions. In our study, 17189 unique peptides were identified which corresponded to 2485 proteins with 2 or more than 2 unique peptides. The identified proteins were then classified per their cellular components, molecular functions, and biological processes by PANTHER [62]. PANTHER classification of the expressed proteins according to biological processes, classified the proteins into 13 groups as shown in Figure 5-3A. The largest group of proteins was cellular processes which included 1131 proteins and contained proteins involved in cell communication (244), cell cycle (116), cellular component movement (52), cytokinesis (23) and growth/proliferation (5). Metabolic processes included 954 proteins. Most of these proteins fell under the subclass of primary metabolic processes (843) and biosynthetic process (95). Cellular component organization and biogenesis was the third largest group with 430 proteins. Localization with 335 proteins included proteins involved in transport. A subclass response to stress, the major subclass under response to stimulus, included 74 proteins. Immune system processes included 33 proteins.

According to the molecular processes, PANTHER classified the expressed proteins into 9 groups as shown in Figure 5-3B. The catalytic activity included the largest group of proteins (705), which consist of following sub-groups: transferase activity (154), oxidoreductase activity (118), hydrolase activity (317), and enzyme regulator activity (59). These groups of molecular processes are important for RPEfunctions. The second largest group was binding (669). Most of the proteins in this group fell under carbohydrate binding (2), chromatin binding (18), nucleic acid binding (232), nucleotide binding (37), antigen binding (1), lipid binding (14), protein binding (382), calcium-dependent phospholipid binding (3), and calcium ion binding (38).

One of the most important functions of RPE is to provide nutrients to the photoreceptors, and thus the proteins corresponding to this function were expected at the output. The bioanalytical platform used in this study, in fact, was able to identify 17 lipids and phospholipids binding proteins. Also, the other groups such as structural molecule activity (173), transporter activity (106), signal transducer activity (47), receptor activity (41), translation regulator activity (31), antioxidant activity (16), and 


\section{A. Biological Processes}

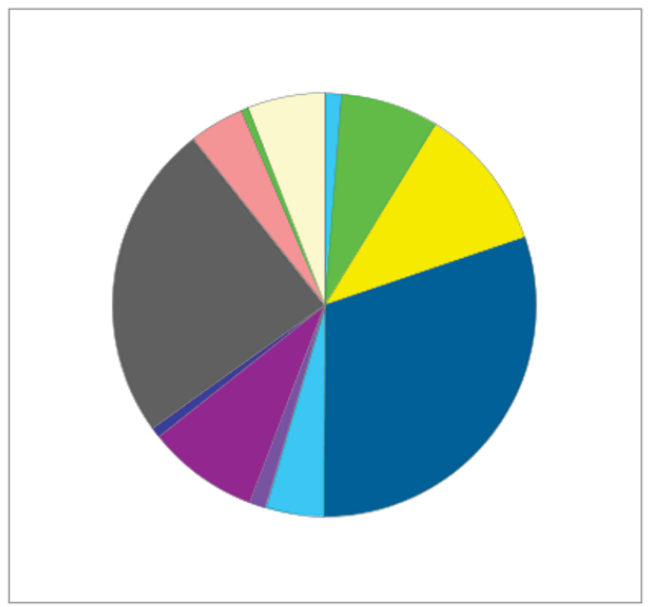

biological adhesion (GO:0022610)- 53

esis (GO:0071840)- 430

cellular component organization or bia
cellular process (GO:0009987)-1131

developmental process (GO:0032502)- 143

growth (GO:0040007)- 2

immune system process (GO:0002376)- 33

localization (GO:0051179)-335

locomotion (GO:0040011)-20

metabolic process (GO:0008152)- 950

multicellular organismal process (GO:0032501)-

reproduction (GO:0000003)- 17

response to stimulus (GO:0050896)- 204

\section{B. Molecular Function}

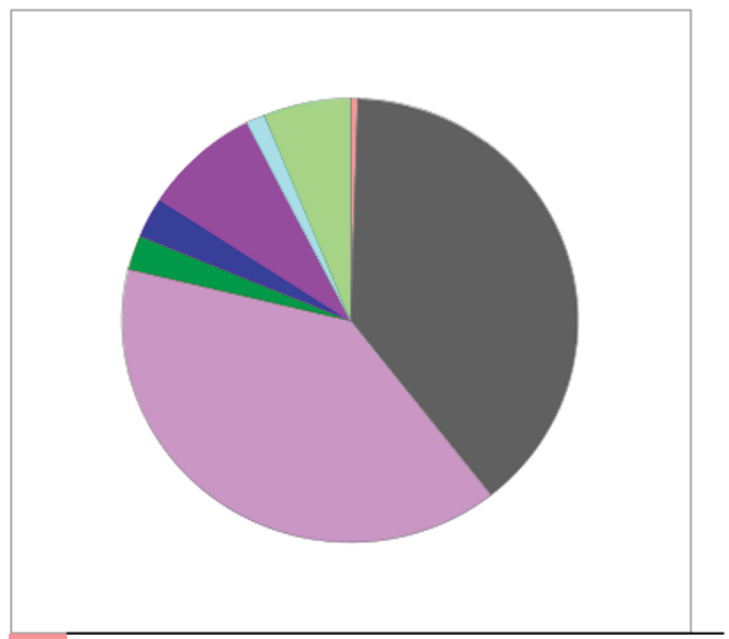

antioxidant activity (GO:0016209)- 16

binding (GO:0005488)- 669

catalytic activity (GO:0003824)- 705

channel regulator activity (GO:0016247)- 1

receptor activity (GO:0004872)-41

signal transducer activity (GO:0004871)-47

structural molecule activity (GO:0005198)-

173

translation regulator activity (GO:0045182)- 31

transporter activity (GO:0005215)-106

\section{Cellular Component}

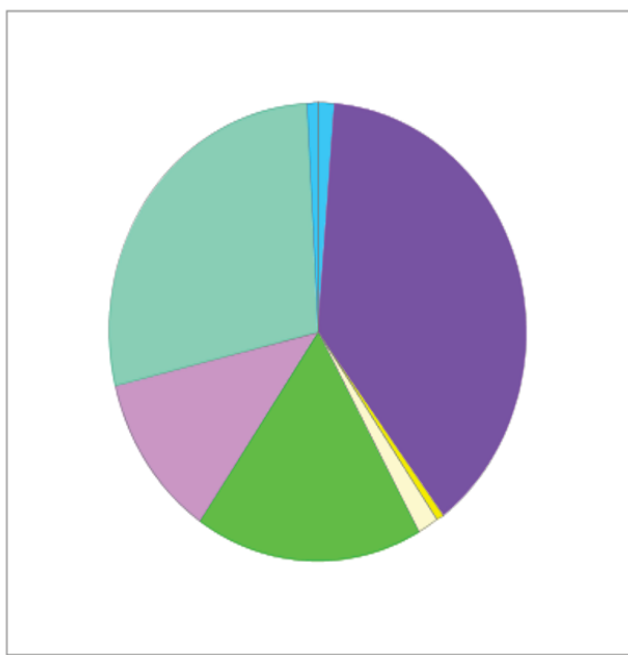

cell junction (GO:0030054)-19

cell part (GO:0044464)-37

extracellular matrix (GO:0031012)-294

extracellular region (GO:0005576)- 15

macromolecular complex (GO:0032991)- 490

membrane (GO:0016020)- 294

organelle (GO:0043226)-730

synapse (GO:0045202)-19

Figure 5-3. Gene ontology categorization of the identified proteins according to (A) biological process, (B). molecular functions and $(\mathrm{C})$. cellular components. 
channel regulator activity (1) are important in RPE functions. In addition to housekeeping genes, a number of proteins known to be associated with RPE functions are identified in this study. RPE cells are the most phagocytic tissue present in humans as they phagocytize the shed outer segment tips from photoreceptors daily. Proteins involved in macromocular degradations such as cathepsin B, Z, D, and L1 were also observed in the result. Furthermore, RPE is rich in reactive oxygen species due to active phagocytosis and photooxidative environment. As expected, the proteins with antioxidant activity are identified and are listed in Table 5-1.

PANTHER classified the identified proteins according to cellular component into 8 groups which are shown in Figure 5-3C. The groups were cell part (1018), organelle (730), macromolecular complex (490), membrane (294), cell junction (37), extracellular matrix (15), and extracellular region (39). RPE monolayer is an integral part of blood retinal barrier, and the integrity of the RPE is critical for retinal function [214]. Proteins corresponding to this are also identified. Specifically, 37 junctional proteins are identified which are important for RPE functions. One of the proteins identified in this study is prominin 1 which is a cholesterol binding protein, and mutated prominin 1 is known to be present in patient with macular degeneration $[215,216]$.

\section{Differential Proteomic Profiling Due to OxLDL Treatment}

The distribution of unique proteins identified in each treatment and the overlap between all the three individual treatments are shown in Figure 5-4. It is noteworthy that even with a large number of proteins identified with the state-of-art high-resolution mass spectrometer, there was a significant overlap among the identified proteins due to the treatments. Specifically, there were 2527 proteins commonly identified in all the treatments, and 83, 196 and 192 proteins uniquely identified in control, LDL and oxLDL treatments respectively.

The oxLDL treatment significantly $(\mathrm{p}=0.05)$ changed the expression of 80 proteins, and among them, 41 proteins were increased/decreased by more than 1.5 -fold due to the treatment. The list of genes with their known function is listed in Table 5-2.

Among the 41 genes, 19 were up-regulated, and 22 were down-regulated. Interestingly, the oxLDL treatment induced the 3.5-fold increase in the expression of HMOX1 which is one of the important components of anti-oxidation system in ARPE-19 cells. The samples were assayed for the upregulation of HMOX1 transcript before performing the proteomic studies on the oxLDL, and LDL treated samples by qPCR. The oxLDL treated samples showed around 40-fold increase and the LDL treated samples showed around 3 fold increase in HMOX1 expression. These results were in line with the transcriptomic data presented in the previous chapter. The other upregulated proteins mostly belong to cell survival, solute carriers. Programmed cell death protein 10 is upregulated important for stability of KDR/VEGFR2. VEGFR2 is under investigations for the wet AMD treatment. Retinoic acid receptor responder (tazarotene induced) 2 
Table 5-1. Proteins involved in antioxidant activity.

\begin{tabular}{ccl}
\hline S.N. & Accession Number & \multicolumn{1}{c}{ Protein Description } \\
\hline 1 & P32119 & Peroxiredoxin-2 \\
2 & Q16881 & Thioredoxin reductase 1, cytoplasmic \\
3 & P78417 & Glutathione S-transferase omega-1 \\
4 & P30044 & Peroxiredoxin-5, mitochondrial \\
5 & P30041 & Peroxiredoxin-6 \\
6 & P04179 & Superoxide dismutase [Mn], mitochondrial \\
7 & P30048 & Thioredoxin-dependent peroxide reductase, mitochondrial \\
8 & P00441 & Superoxide dismutase [Cu-Zn] \\
9 & P07203 & Glutathione peroxidase 1 \\
10 & Q92626 & Peroxidasin homolog \\
11 & Q06830 & Peroxiredoxin-1 \\
12 & O14880 & Microsomal glutathione S-transferase 3 \\
13 & P04040 & Catalase \\
14 & Q8TED1 & Probable glutathione peroxidase 8 \\
15 & Q13162 & Peroxiredoxin-4 \\
16 & P09601 & Heme oxygenase 1 \\
\hline
\end{tabular}




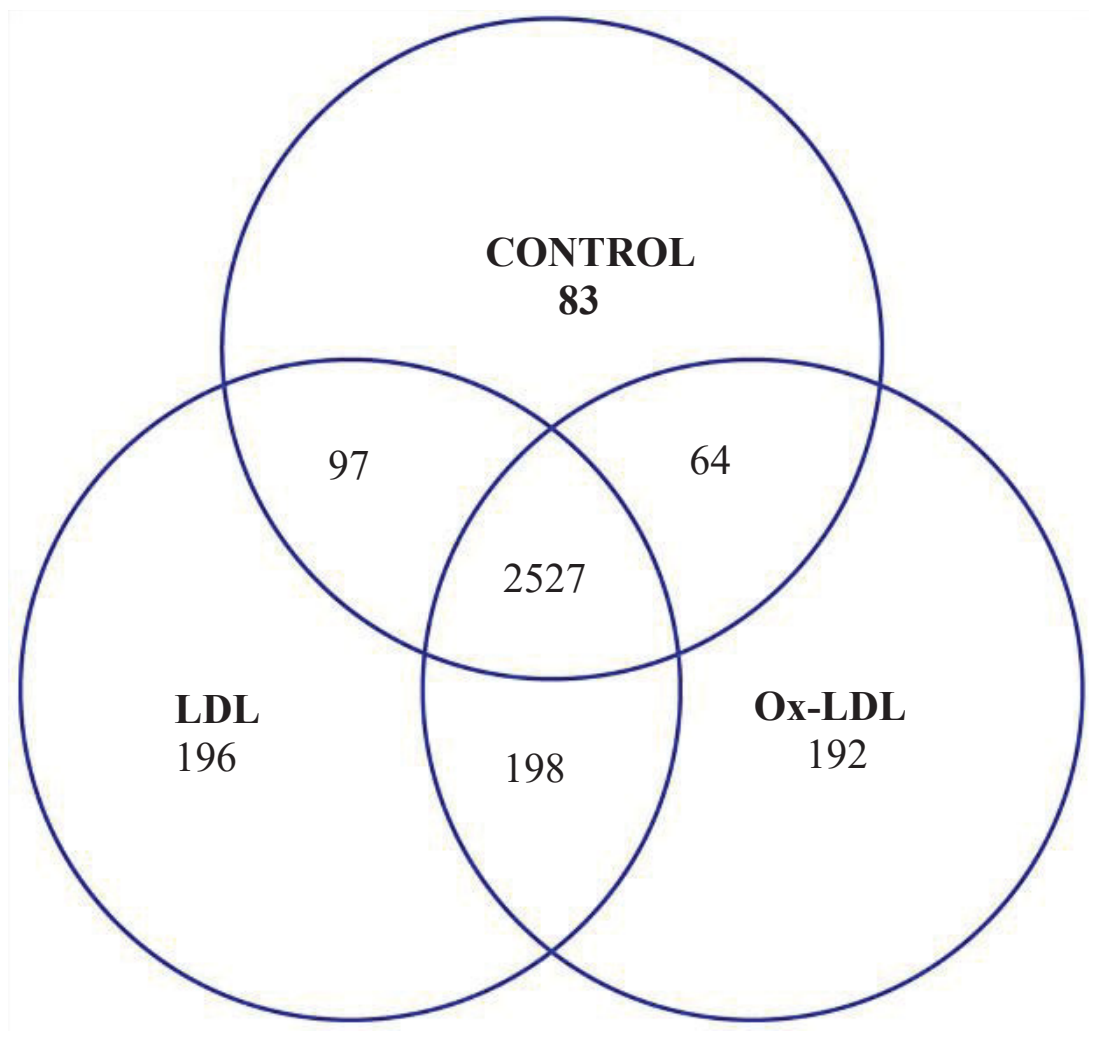

Figure 5-4. Venn diagram of proteins identified in ARPE-19 cells with control, LDL and oxLDL treatments. 
Table 5-2. Differentially expressed proteins due to oxLDL treatment in ARPE-19 cells.

\begin{tabular}{|c|c|c|c|c|c|c|c|}
\hline Accession & $\begin{array}{c}\text { Peptide } \\
\text { count }\end{array}$ & $\begin{array}{c}\text { Confid- } \\
\text { ence } \\
\text { score }\end{array}$ & Anova (p) & $\begin{array}{l}\text { Max fold } \\
\text { change }\end{array}$ & $\begin{array}{c}\text { Highest } \\
\text { mean } \\
\text { condition }\end{array}$ & Description & $\begin{array}{l}\text { Functions } \\
\end{array}$ \\
\hline Q8N1V2 & 5 & 25.1 & 0.00138 & 5.8 & oxLDL & Cilia- and flagella-associated protein 52 & May play a role in cell growth and/or survival \\
\hline P09601 & 11 & 101.4 & 7.30E05 & 3.5 & oxLDL & Heme oxygenase 1 & Exhibits cytoprotective effects \\
\hline P22735 & 2 & 9.5 & 0.04250 & 2.5 & oxLDL & $\begin{array}{l}\text { Protein-glutamine gamma- } \\
\text { glutamyltransferase K }\end{array}$ & Involved in cell proliferation \\
\hline Q6ZU15 & 4 & 24.7 & 0.00201 & 2.1 & oxLDL & Septin-14 & --- \\
\hline Q96H55 & 3 & 16.4 & 0.02799 & 2.0 & oxLDL & Unconventional myosin-XIX & It is involved in mitochondrial motility. \\
\hline Q9BUL8 & 8 & 51.0715 & 0.04763 & 1.9 & oxLDL & Programmed cell death protein 10 & $\begin{array}{l}\text { Promotes cell proliferation. Modulates } \\
\text { apoptotic pathways. Increases MAPK activity } \\
\text { and STK } 26 \text { activity. }\end{array}$ \\
\hline Q99969 & 3 & 17.7399 & 0.00498 & 1.8 & oxLDL & $\begin{array}{l}\text { Retinoic acid receptor responder protein } \\
2\end{array}$ & $\begin{array}{l}\text { Regulates adipogenesis, metabolism and } \\
\text { inflammation }\end{array}$ \\
\hline Q14094 & 4 & 18.0393 & 0.02893 & 1.7 & oxLDL & Cyclin-I & --- \\
\hline Q8NCX0 & 12 & 62.4101 & 0.00817 & 1.7 & oxLDL & $\begin{array}{l}\text { Coiled-coil domain-containing protein } \\
150\end{array}$ & --- \\
\hline Q9ULV0 & 23 & $\begin{array}{c}128.902 \\
8\end{array}$ & 0.00531 & 1.7 & oxLDL & Unconventional myosin- $\mathrm{Vb}$ & $\begin{array}{l}\text { May be involved in vesicular trafficking via the } \\
\text { CART complex that is necessary for efficient } \\
\text { transferrin receptor recycling }\end{array}$ \\
\hline Q06520 & 3 & 15.9978 & 0.03649 & 1.7 & oxLDL & Bile salt sulfotransferase & $\begin{array}{l}\text { Acts as sulfonate donor to catalyze the } \\
\text { sulfonation of steroids and bile acids in the liver } \\
\text { and adrenal glands }\end{array}$ \\
\hline P34059 & 6 & 32.1681 & 0.04384 & 1.6 & oxLDL & $\mathrm{N}$-acetylgalactosamine-6-sulfatase & --- \\
\hline O75027 & 2 & 10.3521 & 0.05771 & 1.6 & oxLDL & $\begin{array}{l}\text { ATP-binding cassette sub-family B } \\
\text { member 7_mitochondrial }\end{array}$ & $\begin{array}{l}\text { Plays a central role in the maturation of } \\
\text { cytosolic iron-sulfur }(\mathrm{Fe} / \mathrm{S}) \text { cluster-containing } \\
\text { proteins. }\end{array}$ \\
\hline P49736 & 13 & 78.4186 & 0.00474 & 1.5 & oxLDL & DNA replication licensing factor MCM2 & $\begin{array}{l}\text { Acts as component of the MCM2-7 complex } \\
\text { (MCM complex) for DNA replication initiation } \\
\text { and elongation in eukaryotic cells. }\end{array}$ \\
\hline P46783 & 12 & 79.0937 & 0.03512 & 1.5 & oxLDL & 40S ribosomal protein $\mathrm{S} 10$ & --- \\
\hline P54802 & 12 & 81.2849 & 0.03310 & 1.5 & oxLDL & Alpha-N-acetylglucosaminidase & Involved in the degradation of heparan sulfate. \\
\hline Q08209 & 8 & 41.0657 & 0.03026 & 1.5 & oxLDL & $\begin{array}{l}\text { Serine/threonine-protein phosphatase } 2 \mathrm{~B} \\
\text { catalytic subunit alpha isoform }\end{array}$ & --- \\
\hline
\end{tabular}


Table 5-2. (Continued.)

\begin{tabular}{|c|c|c|c|c|c|c|c|}
\hline Accession & $\begin{array}{c}\text { Peptide } \\
\text { count }\end{array}$ & $\begin{array}{l}\text { Confid- } \\
\text { ence } \\
\text { score }\end{array}$ & Anova (p) & $\begin{array}{c}\text { Max fold } \\
\text { change }\end{array}$ & $\begin{array}{c}\begin{array}{c}\text { Highest } \\
\text { mean } \\
\text { condition }\end{array} \\
\end{array}$ & $\begin{array}{c}\text { Description } \\
\end{array}$ & $\begin{array}{c}\text { Functions } \\
\end{array}$ \\
\hline Q9BVP2 & 18 & 98.075 & 0.04319 & 1.5 & oxLDL & $\begin{array}{l}\text { Guanine nucleotide-binding protein-like } \\
3\end{array}$ & $\begin{array}{l}\text { May be required to maintain the proliferative } \\
\text { capacity of stem cells. }\end{array}$ \\
\hline Q9Y2V2 & 1 & 6.639 & 0.05232 & 1.5 & oxLDL & Calcium-regulated heat-stable protein 1 & $\begin{array}{l}\text { Binds mRNA and regulates the stability of } \\
\text { target mRNA. Binds single-stranded DNA (in } \\
\text { vitro) }\end{array}$ \\
\hline O75844 & 6 & 35.4133 & 0.04309 & 3.1 & Controls & CAAX prenyl protease 1 homolog & $\begin{array}{l}\text { Proteolytically removes the } \mathrm{C} \text {-terminal three } \\
\text { residues of farnesylated proteins. }\end{array}$ \\
\hline Q13526 & 5 & 27.5287 & 0.041241 & 2.7 & Controls & $\begin{array}{l}\text { Peptidyl-prolyl cis-trans isomerase } \\
\text { NIMA-interacting } 1\end{array}$ & $\begin{array}{l}\text { Regulates mitosis presumably by interacting } \\
\text { with NIMA and attenuating its mitosis- } \\
\text { promoting activity. }\end{array}$ \\
\hline Q5TF21 & 4 & 26.0579 & 0.004162 & 2.7 & Controls & Protein SOGA3 & --- \\
\hline P55011 & 6 & 28.7042 & 0.014657 & 2.4 & Controls & Solute carrier family 12 member 2 & $\begin{array}{l}\text { Mediates sodium and chloride reabsorption. } \\
\text { Plays a vital role in the regulation of ionic } \\
\text { balance and cell volume. }\end{array}$ \\
\hline Q5T7M9 & 5 & 31.696 & 0.030891 & 2.2 & Controls & Protein FAM69A & --- \\
\hline Q96G01 & 26 & $\begin{array}{c}149.339 \\
4\end{array}$ & 0.045389 & 2.2 & Controls & Protein bicaudal D homolog 1 & $\begin{array}{l}\text { Regulates coat complex coatomer protein I } \\
\text { (COPI)- independent Golgi-endoplasmic } \\
\text { reticulum transport by recruiting the dynein- } \\
\text { dynactin motor complex. }\end{array}$ \\
\hline Q6IPM2 & 7 & 45.8517 & 0.022053 & 2.1 & Controls & IQ domain-containing protein $\mathrm{E}$ & --- \\
\hline P18827 & 2 & 11.8076 & 0.033626 & 2.1 & Controls & Syndecan-1 & $\begin{array}{l}\text { Regulates exosome biogenesis in concert with } \\
\text { SDCBP and PDCD6IP. }\end{array}$ \\
\hline Q9H4A6 & 6 & 34.4795 & 0.042464 & 2.0 & Controls & Golgi phosphoprotein 3 & $\begin{array}{l}\text { Phosphatidylinositol-4-phosphate-binding } \\
\text { protein that links Golgi membranes to the } \\
\text { cytoskeleton }\end{array}$ \\
\hline Q9BQE5 & 5 & 29.7731 & 0.016539 & 2.0 & Controls & Apolipoprotein L2 & $\begin{array}{l}\text { May affect the movement of lipids in the } \\
\text { cytoplasm or allow the binding of lipids to } \\
\text { organelles }\end{array}$ \\
\hline Q96KN1 & 7 & 51.3589 & 0.022464 & 1.5 & Controls & Protein FAM84B & --- \\
\hline
\end{tabular}


Table 5-2. (Continued.)

\begin{tabular}{|c|c|c|c|c|c|c|c|}
\hline Accession & $\begin{array}{c}\text { Peptide } \\
\text { count }\end{array}$ & $\begin{array}{c}\text { Confid- } \\
\text { ence } \\
\text { score } \\
\end{array}$ & Anova (p) & $\begin{array}{c}\text { Max fold } \\
\text { change }\end{array}$ & $\begin{array}{c}\text { Highest } \\
\text { mean } \\
\text { condition }\end{array}$ & $\begin{array}{c}\text { Description } \\
\end{array}$ & $\begin{array}{c}\text { Functions } \\
\end{array}$ \\
\hline Q5T200 & 17 & 91.7961 & 0.027959 & 1.9 & Controls & $\begin{array}{l}\text { Zinc finger } \mathrm{CCCH} \text { domain-containing } \\
\text { protein } 13\end{array}$ & $\begin{array}{l}\text { Acts as component of the WTAP complex that } \\
\text { is involved in RNA processing and cell cycle }\end{array}$ \\
\hline Q8NFU7 & 2 & 11.8539 & 0.034706 & 1.9 & Controls & Methylcytosine dioxygenase TET1 & Plays a key role in active DNA demethylation. \\
\hline Q8TES7 & 2 & 9.115 & 0.034421 & 1.7 & Controls & Fas-binding factor 1 & $\begin{array}{l}\text { Keratin-binding protein required for epithelial } \\
\text { cell polarization. Involved in apical junction } \\
\text { complex (AJC) assembly via its interaction } \\
\text { with PARD3. Required for ciliogenesis }\end{array}$ \\
\hline Q9H3P7 & 15 & 84.7999 & 0.033429 & 1.7 & Controls & Golgi resident protein GCP60 & $\begin{array}{l}\text { Involved in the maintenance of Golgi structure } \\
\text { by interacting with giantin, affecting protein } \\
\text { transport between the endoplasmic reticulum } \\
\text { and Golgi. }\end{array}$ \\
\hline Q9Y697 & 7 & 36.5836 & 0.029239 & 1.7 & Controls & Cysteine desulfurase_mitochondrial & $\begin{array}{l}\text { Catalyzes the removal of elemental sulfur from } \\
\text { cysteine to produce alanine. It supplies the } \\
\text { inorganic sulfur for iron- sulfur (Fe-S) clusters. }\end{array}$ \\
\hline Q8NDZ4 & 10 & 55.4458 & 0.012477 & 1.6 & Controls & Deleted in autism protein 1 & $\begin{array}{l}\text { May play a role in cardiomyocyte proliferation } \\
\text { through paracrine signaling and activation of } \\
\text { the PPI3K-AKT-CDK } 7 \text { signaling cascade }\end{array}$ \\
\hline $\begin{array}{l}\mathrm{P} 61513 / \mathrm{A} \\
6 \mathrm{NKH3}\end{array}$ & 2 & 12.164 & 0.052936 & 1.5 & Controls & $60 \mathrm{~S}$ ribosomal protein $\mathrm{L} 37 \mathrm{a}$ & --- \\
\hline O43264 & 11 & 57.0269 & 0.023874 & 1.5 & Controls & $\begin{array}{l}\text { Centromere/kinetochore protein zw10 } \\
\text { homolog }\end{array}$ & $\begin{array}{l}\text { Essential component of the mitotic checkpoint, } \\
\text { which prevents cells from prematurely exiting } \\
\text { mitosis }\end{array}$ \\
\hline Q86YR7 & 13 & 70.7216 & 0.004251 & 1.5 & Controls & $\begin{array}{l}\text { Probable guanine nucleotide exchange } \\
\text { factor MCF2L2 }\end{array}$ & --- \\
\hline Q8IWB7 & 15 & 89.8162 & 0.043805 & 1.5 & Controls & $\begin{array}{l}\text { WD repeat and FYVE domain-containing } \\
\text { protein } 1\end{array}$ & $\begin{array}{l}\text { Positively regulates TLR3- and TLR4-mediated } \\
\text { signaling pathways. Promotes TLR3/4 ligand- } \\
\text { induced activation of transcription factors IRF3 } \\
\text { and NF-kappa-B, as well as the production of } \\
\text { IFN-beta and inflammatory cytokines. }\end{array}$ \\
\hline Q96IZ0 & 7 & 41.9123 & 0.043278 & 1.5 & Controls & PRKC apoptosis WT1 regulator protein & $\begin{array}{l}\text { Pro-apoptopic protein capable of selectively } \\
\text { inducing apoptosis in cancer cells. }\end{array}$ \\
\hline
\end{tabular}

Source: https://string-db.org/cgi/input.pl? sessionId=K834LhgFlCOW\&input page show search=on 
protein is also induced by due to oxLDL treatment which plays role in inflammation through activation of the chemokine-like receptor 1 (CMKLR1). Among the down regulated genes, Apolipoprotein L is downregulated 2 fold. This protein plays an important role in cholesterol transport and also affects the movement of lipids in the cytoplasm. It also allows the binding of lipids to organelles.

\section{Transcriptomic Versus Proteomic Analysis}

The transcriptomic data from the oxLDL treatment, described in the previous chapter, was compared with the proteomic data obtained in this study. Precisely, the oxLDL induced gene expression data from microarrays that increased/decreased at least two folds after oxLDL treatments were compared with the list of proteome qualitative data. Among the 153 differentially expressed genes in transcriptome analysis, 22 were probed in the qualitative proteome data. The rest were not probed by proteomic analysis Similarly, among the 41 differentially expressed proteins in proteome analysis, all of them were probed in the transcriptome data (Table 5-3). Also, when differentially expressed genes were compared to differentially expressed proteins only two genes were common to both the list. HMOX1 is upregulated in both the transcriptome and proteome data whereas BICD1 is upregulated in transcriptome data and downregulated in proteome data. While the proteome data did not show comparable fold changes from oxLDL VS control groups as it had shown in transcriptome data, the trend of regulation was similar for most of the proteins. The very subtle changes seen in the proteome study may be due to the short incubation time that might not have given sufficient time for the changes in genes to translate into changes into proteins. This observation was similar to the observations reported by many other studies, i.e., they also compared the transcriptome data with corresponding proteome data from the same sample and under the same condition and couldn't get a high correlation between the two [217-221]. Various other reasons could explain the discrepancy between the transcriptome data with the proteome data. First, the half-life of mRNA is less compared to protein half-life [222]. Moreover, various post-translational changes also result in the decreased half-life of the proteins [25] or even affect the steady-state level of protein in a given tissue at a particular time. Also, the unpredictability of mRNA expression level during cell cycle can affect the mRNA-protein correlation [223].

There exists a study that compared the transcriptome and proteome changes in ARPE-19 cells due to photocoagulation. In this study, the authors measured the transcriptome at $2 \mathrm{~h}, 6 \mathrm{~h}$, and $24 \mathrm{~h}$, whereas proteome analysis was only done after $48 \mathrm{~h}$. With the Still, the authors found only 24 common genes, out of more than 900 genes differentially expressed genes on microarray data that showed changes in proteome level [224]. This aligns with the very little correlation of transcriptome and proteome data in our samples as our analysis of proteome and transcriptome data was done at the same time point i.e.at $4 \mathrm{~h}$.

We also analyzed our list of differentially expressed genes that were involved in the different molecular pathways described in chapter 4. Among the genes involved in 
Table 5-3. List of common proteins/genes probed in microarray and proteomic studies.

\begin{tabular}{|c|c|c|}
\hline Gene Name & UniProt ID & Description \\
\hline HMOX1 & P09601 & Heme oxygenase 1 \\
\hline CPT1A & P50416 & Carnitine O-palmitoyltransferase 1 _ liver isoform \\
\hline CTGF & P29279 & Connective tissue growth factor \\
\hline EHD4 & Q9H223 & EH domain-containing protein 4 \\
\hline ENO2 & P09104 & Gamma-enolase \\
\hline EPHA2 & P29317;P29322;P54756 & Ephrin type-A receptor \\
\hline EPHX1 & P07099 & Epoxide hydrolase 1 \\
\hline FASN & P49327 & Fatty acid synthase \\
\hline HK2 & P52789 & Hexokinase-2 \\
\hline HMGCS1 & Q01581 & Hydroxymethylglutaryl-CoA synthase_cytoplasmic \\
\hline JAK2 & O60674 & Tyrosine-protein kinase JAK2 \\
\hline LDLR & Q14696 & LDLR chaperone MESD \\
\hline LPCAT1 & Q8NF37 & Lysophosphatidylcholine acyltransferase 1 \\
\hline MPP2 & Q14168 & MAGUK p55 subfamily member 2 \\
\hline NQO1 & P15559 & $\mathrm{NAD}(\mathrm{P}) \mathrm{H}$ dehydrogenase [quinone] 1 \\
\hline PCDH18 & Q9HCL0 & Protocadherin-18 \\
\hline PHLPP1 & O60346 & PH domain leucine-rich repeat-containing protein phosphatase 1 \\
\hline PLIN2 & Q99541 & Perilipin-2 \\
\hline SLC3A2 & P08195 & $4 \mathrm{~F} 2$ cell-surface antigen heavy chain \\
\hline SPTLC2 & $\mathrm{O} 15270$ & Serine palmitoyltransferase 2 \\
\hline TXNIP & Q9H3M7 & Thioredoxin-interacting protein \\
\hline CFAP52 & Q8N1V2 & Cilia- and flagella-associated protein \\
\hline TGM1 & $\mathrm{P} 22735$ & Protein-glutamine gamma-glutamyltransferase $\mathrm{K}$ \\
\hline 14-Sep & Q6ZU15 & Septin-14 \\
\hline MYO19 & Q96H55 & Unconventional myosin-XIX \\
\hline PDCD10 & Q9BUL8 & Programmed cell death protein 10 \\
\hline RARRES2 & Q99969 & Retinoic acid receptor responder protein 2 \\
\hline CCNI & Q14094 & Cyclin-I \\
\hline $\mathrm{CCDC} 150$ & Q8NCX0 & Coiled-coil domain-containing protein 150 \\
\hline MYO5B & Q9ULV0 & Unconventional myosin- $\mathrm{Vb}$ \\
\hline SULT2A1 & Q06520 & Bile salt sulfotransferase \\
\hline GALNS & P34059 & $\mathrm{N}$-acetylgalactosamine-6-sulfatase \\
\hline $\mathrm{ABCB} 7$ & O75027 & ATP-binding cassette sub-family B member 7_mitochondrial \\
\hline MCM2 & P49736 & DNA replication licensing factor MCM2 \\
\hline RPS10 & P46783 & $40 \mathrm{~S}$ ribosomal protein $\mathrm{S} 10$ \\
\hline NAGLU & P54802 & Alpha-N-acetylglucosaminidase \\
\hline PPP3CA & Q08209 & Serine/threonine-protein phosphatase $2 \mathrm{~B}$ catalytic subunit alpha \\
\hline GNL3 & Q9BVP2 & Guanine nucleotide-binding protein-like 3 \\
\hline CARHSP1 & Q9Y2V2 & Calcium-regulated heat-stable protein 1 \\
\hline ZMPSTE24 & O75844 & CAAX prenyl protease 1 homolog \\
\hline
\end{tabular}


Table 5-3. (Continued.)

\begin{tabular}{lcl}
\hline \multicolumn{1}{c}{ Gene Name } & UniProt ID & \multicolumn{1}{c}{ Description } \\
\hline PIN1 & Q13526 & Peptidyl-prolyl cis-trans isomerase NIMA-interacting 1 \\
SOGA3 & Q5TF21 & Protein SOGA3 \\
SLC12A2 & P55011 & Solute carrier family 12 member 2 \\
FAM69A & Q5T7M9 & Protein FAM69A \\
BICD1 & Q96G01 & Protein bicaudal D homolog 1 \\
IQCE & Q6IPM2 & IQ domain-containing protein E \\
SDC1 & P18827 & Syndecan-1 \\
GOLPH3 & Q9H4A6 & Golgi phosphoprotein 3 \\
APOL2 & Q9BQE5 & Apolipoprotein L2 \\
ZC3H13 & Q5T200 & Zinc finger CCCH domain-containing protein 13 \\
TET1 & Q8NFU7 & Methylcytosine dioxygenase TET1 \\
FBF1 & Q8TES7 & Fas-binding factor 1 \\
ACBD3 & Q9H3P7 & Golgi resident protein GCP60 \\
NFS1 & Q9Y697 & Cysteine desulfurase_ mitochondrial \\
C3orf58 & Q8NDZ4 & Deleted in autism protein 1 \\
RPL37A & P61513;A6NKH3 & 60S ribosomal protein L37a \\
ZW10 & O43264 & Centromere/kinetochore protein zw10 homolog \\
MCF2L2 & Q86YR7 & Probable guanine nucleotide exchange factor MCF2L2 \\
FAM84B & Q96KN1 & Protein FAM84B \\
WDFY1 & Q8IWB7 & WD repeat and FYVE domain-containing protein 1 \\
PAWR & Q96IZ0 & PRKC apoptosis WT1 regulator protein \\
\hline
\end{tabular}


anti-oxidative mechanism, lipid metabolism, autophagy and circadian rhythm eight genes were probed in qualitative proteome list: HMOX1, FASN, HMGCS1, LDLR, NQO1, PHLPP1, SPTLC2, and TXNIP. The proteins involved in oxidative stress are HMOX1, TXNIP and NQO1, similarly proteins involved in lipid metabolism were LDLR, HMGCS1, FASN and SPTLC2. Only gene involved in circadian rhythm was identified in qualitative proteome list i.e. PHLPP1.

\section{Conclusion}

Mass spectrometry based proteomics has been a work horse for discovery-based research in the field of proteomics. The development of new hybrid instruments and various sample preparation and separation techniques along with data acquisition methods has fundamentally changed the way scientists interrogate biological systems. The MS based proteomics has outstanding ability to investigate thousands of proteins along with post translational modification qualitatively and quantitatively.

In our study, we utilized a bioanalytical platform that included nano-UPLC along with state-of-art mass spectrometer to investigate the proteome of ARPE-19 cells. We also interrogated oxLDL mediated differential proteome alteration in ARPE-19 cells., the first study of its kind in the literature. The use of nano UPLC increased separation efficiency, improved resolution, decreased the analysis time and lowered the operating cost when compared to the nano HPLC used in our previous study (Chapter 2). Higher chromatographic separation and mass resolution provided high coverage and mass spectral data quality. High quality of mass spectral data is necessary for quantitative analysis. The current bioanalytical platform outperformed the bioanalytical platform discussed in chapter 2 in terms of proteome coverage. The new platform was able to identify 3-fold more proteins compared to the best described in chapter 2. Moreover, the ARPE-19 protein panel encompassed proteins with various functional roles including macromolecular degradation and anti-oxidant activity and junctional proteins. The bioanalytical platform used in this study utilized data independent acquisition (DIA) method which allowed the fragmentation and identification of all the peptides that eluted at a given point in gradient elution. Hence, it provided unbiased identification and label free quantification of protein in the oxLDL treated and untreated samples.

Interestingly, there was very little correlation between the $4 \mathrm{~h}$ transcriptome and proteome data as seen by various other studies. This study provides the foundation of a bioanalytical platform for identifying the RPE proteome. The list of differentially expressed proteins due to oxLDL treatment identified in this study gives insight to the change in proteins that are applicable in terms of implication for AMD. 


\section{CHAPTER 6. CONCLUSION AND FUTURE DIRECTIONS}

Mass spectrometry based proteomics is an important component of biological research today as it allows identification, quantification, and characterization of proteins to better understand the complex biological pathways and protein-protein interactions undergoing within the cells. Both data-dependent and data-independent mass spectrometry based approaches are continuously being optimized and improved which enable scientists in obtaining finest results in terms of the desired outcome.

Towards the data-dependent approach in mass spectrometry, we presented a bioanalytical platform, a bottom-up approach that was able to provide better coverage and identification of proteins in a biological system. Three different bio-analytical platforms that utilized IEF, SDS-PAGE, and RP-RP as pre-fractionation methods before LC-MS/MS analysis were evaluated. Among the three platforms that were used in our study, the SDS-PAGE-LC-MS/MS was superior to the IEF and RP-RP separation in terms of in-depth protein coverage. We also leveraged the data-independent approach for data acquisition on a high-resolution mass spectrometry, a more recent method in bottom up proteomics, to identify and quantify protein profile in ARPE-19 cells. On this regard, we showed that the use of high-resolution mass spectrometry even without the fractionation provides the higher number of protein identification and label-free quantification. The use of nano-UPLC, high-resolution instrument and data independent acquisition method alone increased the protein identification from 980 protein obtained from pre-fractionation of samples to $c a$. 3000 proteins without fractionation. In addition to proteome profiling, the bioanalytical platform was able to identify proteins with various functional roles.

Both the proteomics and transcriptomic based application approaches were applied to understand the oxidized lipoproteins- induced changes in the ARPE-19 cells to better understand the molecular mechanism that may be contributing to development of AMD, which is the leading cause of blindness in the western world. The transcriptome analysis of early events showed the involvement of Nrf- 2 mediated pathways and the induction of anti-oxidative pathways to protect the cells from the oxidative damage. In addition to the induction of genes involved in the protection of cells against the oxidative stress the study also showed the downregulation of genes involved in lipoprotein uptake and cholesterol biosynthesis. One of the novel pathways discovered in our studies is the circadian rhythm pathways. The genes involved in circadian rhythm were down-regulated due to oxLDL treatment for four hours. In an analogous study to the transcriptome analysis, we performed proteome analysis of the oxLDL induced changes in ARPE-19 cells which also showed the upregulation of anti-oxidant HMOX1 similar to the transcriptome results. However, the amount and extent of change that we saw in the transcriptome did not translate to the proteome as indicated by many other studies [147149]. For future continuation of this study, bioanalytical and biological considerations can be made. To obtain better correlation between transcriptome and proteome data, it would be interesting to see the effect of using of extra-long gradients with/without prefractionation prior to LC-MS/MS analysis. For biological consideration longer oxLDL 
exposures can be done to get mechanistic understanding of the differentially expressed proteins.

Accumulated oxidized lipids play a crucial role in the development of AMD which are identified by scavenger receptors present in the RPE cells. The scavenger receptors aid in clearance of oxidized lipids by these cells. In our study, we studied the role of scavenger receptors CD36 and CD5L/AIM in oxLDL uptake by ARPE-19 cells. The scavenger receptor CD5L/AIM was recently identified as an increased autoantigen in the diseased population by our group. We demonstrated that ARPE-19 cells internalize oxLDL which was primarily dependent on CD36 because the use of SSO which binds with CD36 significantly decreased the uptake of oxLDL in the cells. We also demonstrated that oxLDL does bind to CD5L/AIM by ELISA and also in-vivo by live imaging with CD5L/AIM transfected cells. Moreover, the CD5L/AIM and CD36 were seen to co-localize in the presence of oxLDL. However, our results did not show the change in CD5L/AIM and CD36 in the transcript or in the protein level after 4 hours of oxLDL treatment. The use of anti- CD5L/AIM antibody did show a reduction of the amount of intracellular oxLDL in the ARPE-19 cells which was, however, not significant when compared with the isotype control. Therefore, to see the effect of adding external CD5L/AIM on the oxLDL clearance by the cells, we incubated the cells with DiI-oxLDL in the presence of rhCD5L/AIM. This study, which involved measurement of intracellular oxLDL at a single time point (after 5h of oxLDL exposure) showed a significant reduction of the accumulation of intracellular oxLDL in ARPE-19 cells.

The reduction in intracellular accumulation of oxLDL due to extracellular recombinant CD5L/AIM is an interesting phenomenon as it has been recently shown the involvement of CD5L in autophagy. It would be interesting to know how the CD5L/AIM works in reducing accumulation of oxLDL in the cells. Does CD5L/AIM block the uptake of oxLDL by itself or does it aid in early clearance of oxLDL by ARPE-19 cells? Answers to these questions would elucidate the innovative role of CD5L/AIM in oxLDL clearance in the eye. For this, the use of the antibody that could bind to the rhCD5L or IgM which is known to bind to the CD5L/AIM could elucidate the mechanism. Moreover, a study of intracellular oxLDL accumulation over multiple exposure times would shed more light into the dynamics of oxLDL degradation by the cells and help understand the role of CD5L/AIM in this process. Finally, to understand the involvement of CD5L/AIM in autophagy-related processes the use of autophagy inhibitor along with rh CD5L/AIM would validate the role of rh CD5L/AIM in intracellular oxLDL clearance by ARPE-19 cells. 


\section{LIST OF REFERENCES}

1. Whitcup, S.M., et al., The role of the immune response in age-related macular degeneration. Int J Inflam, 2013. 2013: p. 348092.

2. O., S., The Retinal Pigment Epithelium. Webvision: The Organization of the Retina and Visual System [Internet], ed. F.E. Kolb H, Nelson R. 2011 Jan 26, Salt Lake City (UT): University of Utah Health Sciences Center.

3. Curcio, C.A., et al., The oil spill in ageing Bruch membrane. Br J Ophthalmol, 2011. 95(12): p. 1638-45.

4. Delori, F.C., D.G. Goger, and C.K. Dorey, Age-related accumulation and spatial distribution of lipofuscin in RPE of normal subjects. Invest Ophthalmol Vis Sci, 2001. 42(8): p. 1855-66.

5. Boulton, M. and P. Dayhaw-Barker, The role of the retinal pigment epithelium: topographical variation and ageing changes. Eye (Lond), 2001. 15(Pt 3): p. 3849.

6. Itabe, H., T. Obama, and R. Kato, The Dynamics of Oxidized LDL during Atherogenesis. J Lipids, 2011. 2011: p. 418313.

7. Wilkins, M.R., et al., Progress with proteome projects: Why all proteins expressed by a genome should be identified and how to do it. Biotechnology and Genetic Engineering Reviews, Vol 13, 1996. 13: p. 19-50.

8. Kim, M.S., et al., A draft map of the human proteome. Nature, 2014. 509(7502): p. $575-+$.

9. Domon, B. and R. Aebersold, Mass spectrometry and protein analysis. Science, 2006. 312(5771): p. 212-7.

10. Aebersold, R. and M. Mann, Mass-spectrometric exploration of proteome structure and function. Nature, 2016. 537(7620): p. 347-355.

11. Baginsky, S., et al., Gene Expression Analysis, Proteomics, and Network Discovery. Plant Physiology, 2010. 152(2): p. 402-410.

12. Godovac-Zimmermann, J. and L.R. Brown, Perspectives for mass spectrometry and functional proteomics. Mass Spectrometry Reviews, 2001. 20(1): p. 1-57.

13. Lamond, A.I., Molecular biology of the cell, 4th edition. Nature, 2002. 417(6887): p. 383-383.

14. Chandramouli, K. and P.Y. Qian, Proteomics: challenges, techniques and possibilities to overcome biological sample complexity. Hum Genomics Proteomics, 2009. 2009.

15. Cravatt, B.F., G.M. Simon, and J.R. Yates, 3rd, The biological impact of massspectrometry-based proteomics. Nature, 2007. 450(7172): p. 991-1000.

16. Toby, T.K., L. Fornelli, and N.L. Kelleher, Progress in Top-Down Proteomics and the Analysis of Proteoforms. Annual Review of Analytical Chemistry, Vol 9, 2016. 9: p. 499-519.

17. Carr, S.A., et al., Targeted Peptide Measurements in Biology and Medicine: Best Practices for Mass Spectrometry- based Assay Development Using a Fit-forPurpose Approach. Molecular \& Cellular Proteomics, 2014. 13(3): p. 907-917.

18. Crutchfield, C.A., et al., Advances in mass spectrometry-based clinical biomarker discovery. Clinical Proteomics, 2016. 13. 
19. Giorgianni, F., et al., Proteome analysis of subsarcolemmal cardiomyocyte

mitochondria: a comparison of different analytical platforms. Int J Mol Sci, 2014. 15(6): p. 9285-301.

20. Yates, J.R. and N.L. Kelleher, Top Down Proteomics. Analytical Chemistry, 2013. 85(13): p. 6151-6151.

21. Zhang, Y.Y., et al., Protein Analysis by Shotgun/Bottom-up Proteomics. Chemical Reviews, 2013. 113(4): p. 2343-2394.

22. Wisniewski, J.R., et al., Universal sample preparation method for proteome analysis. Nature Methods, 2009. 6(5): p. 359-U60.

23. Smejkal, G.B., et al., Sample preparation for two-dimensional gel electrophoresis using pressure cycling technology. Analytical Biochemistry, 2007. 363(2): p. 309311.

24. Kleparnik, K. and P. Bocek, Electrophoresis today and tomorrow: helping biologists' dreams come true. Bioessays, 2010. 32(3): p. 218-226.

25. Rabilloud, T. and C. Lelong, Two-dimensional gel electrophoresis in proteomics: a tutorial. J Proteomics, 2011. 74(10): p. 1829-41.

26. Finoulst, I., et al., Sample Preparation Techniques for the Untargeted LC-MSBased Discovery of Peptides in Complex Biological Matrices. Journal of Biomedicine and Biotechnology, 2011.

27. Diez, R., et al., 2-D Fluorescence Difference Gel Electrophoresis (DIGE) in Neuroproteomics, in Neuroproteomics, O. Alzate, Editor. 2010: Boca Raton (FL).

28. Dzieciatkowska, M., R. Hill, and K.C. Hansen, GeLC-MS/MS analysis of complex protein mixtures. Methods Mol Biol, 2014. 1156: p. 53-66.

29. Paulo, J.A., et al., Mass Spectrometry-Based (GeLC-MS/MS) Comparative Proteomic Analysis of Endoscopically (ePFT) Collected Pancreatic and Gastroduodenal Fluids. Clinical and Translational Gastroenterology, 2012. 3.

30. Piersma, S.R., et al., Whole gel processing procedure for GeLC-MS/MS based proteomics. Proteome Science, 2013. 11.

31. Broeckx, V., et al., Comparison of multiple protein extraction buffers for GeLCMS/MS proteomic analysis of liver and colon formalin-fixed, paraffin-embedded tissues. Molecular Biosystems, 2016. 12(2): p. 553-565.

32. Giorgianni, F., D.M. Desiderio, and S. Beranova-Giorgianni, Proteome analysis using isoelectric focusing in immobilized $\mathrm{pH}$ gradient gels followed by mass spectrometry. Electrophoresis, 2003. 24(1-2): p. 253-259.

33. Beranova-Giorgianni, S., D.M. Desiderio, and F. Giorgianni, Phosphoproteome analysis by in-gel isoelectric focusing and tandem mass spectrometry. Methods Mol Biol, 2009. 519: p. 383-96.

34. Chen, L., F. Giorgianni, and S. Beranova-Giorgianni, Characterization of the Phosphoproteome in LNCaP Prostate Cancer Cells by In-Gel Isoelectric Focusing and Tandem Mass Spectrometry. Journal of Proteome Research, 2010. 9(1): p. 174-178.

35. Beranova-Giorgianni, S., et al., Phosphoproteomic analysis of the human pituitary. Pituitary, 2006. 9(2): p. 109-20.

36. Cornell, F.N., Isoelectric focusing, blotting and probing methods for detection and identification of monoclonal proteins. Clin Biochem Rev, 2009. 30(3): p. 123-30. 
37. Schirmer, E.C., J.R. Yates, 3rd, and L. Gerace, MudPIT: A powerful proteomics tool for discovery. Discov Med, 2003. 3(18): p. 38-9.

38. Law, H.C.H., et al., A versatile reversed phase-strong cation exchange-reversed phase (RP-SCX-RP) multidimensional liquid chromatography platform for qualitative and quantitative shotgun proteomics. Analyst, 2015. 140(4): p. 12371252.

39. McDonald, W.H., et al., Comparison of three directly coupled HPLC MS/MS strategies for identification of proteins from complex mixtures: single-dimension $L C-M S / M S$, 2-phase MudPIT, and 3-phase MudPIT. International Journal of Mass Spectrometry, 2002. 219(1): p. 245-251.

40. Guzzetta, A.W. and A.S. Chien, A double-vented tetraphasic continuous column approach to MuDPIT analysis on long capillary columns demonstrates superior proteomic coverage. Journal of Proteome Research, 2005. 4(6): p. 2412-2419.

41. Gilar, M., et al., Two-dimensional separation of peptides using RP-RP-HPLC system with different $p H$ in first and second separation dimensions. J Sep Sci, 2005. 28(14): p. 1694-703.

42. Gilar, M., et al., Two-dimensional separation of peptides using RP-RP-HPLC system with different $p H$ in first and second separation dimensions. Journal of Separation Science, 2005. 28(14): p. 1694-1703.

43. Nakamura, T., J. Kuromitsu, and Y. Oda, Evaluation of comprehensive multidimensional separations using reversed-phase, reversed-phase liquid chromatography/mass spectrometry for shotgun proteomics. Journal of Proteome Research, 2008. 7(3): p. 1007-1011.

44. Cai, X.M., et al., Two-dimensional liquid chromatography separation of peptides using reversed-phase/weak cation-exchange mixed-mode column in first dimension. Journal of Chromatography A, 2012. 1228: p. 242-249.

45. Wilson, S.R., et al., Nano-LC in proteomics: recent advances and approaches. Bioanalysis, 2015. 7(14): p. 1799-1815.

46. Woods, A.G., et al., Mass Spectrometry for Proteomics-Based Investigation. Advancements of Mass Spectrometry in Biomedical Research, 2014. 806: p. 1-32.

47. Han, X.M., A. Aslanian, and J.R. Yates, Mass spectrometry for proteomics. Current Opinion in Chemical Biology, 2008. 12(5): p. 483-490.

48. Canas, B., et al., Mass spectrometry technologies for proteomics. Brief Funct Genomic Proteomic, 2006. 4(4): p. 295-320.

49. Xu, C.F., et al., Identification of Phosphopeptides by MALDI Q-TOF MS in positive and negative ion modes after methyl esterification. Molecular \& Cellular Proteomics, 2005. 4(6): p. 809-818.

50. Banerjee, S. and S. Mazumdar, Electrospray Ionization Mass Spectrometry: A Technique to Access the Information beyond the Molecular Weight of the Analyte. International Journal of Analytical Chemistry, 2012.

51. Ho, C.S., et al., Electrospray ionisation mass spectrometry: principles and clinical applications. Clin Biochem Rev, 2003. 24(1): p. 3-12.

52. Vatansever, B., et al., Comparison between a linear ion trap and a triple quadruple MS in the sensitive detection of large peptides at femtomole amounts on column. Journal of Separation Science, 2010. 33(16): p. 2478-2488. 
53. Bern, M., et al., Deconvolution of Mixture Spectra from Ion-Trap DataIndependent-Acquisition Tandem Mass Spectrometry. Analytical Chemistry, 2010. 82(3): p. 833-841.

54. Gillet, L.C., A. Leitner, and R. Aebersold, Mass Spectrometry Applied to BottomUp Proteomics: Entering the High-Throughput Era for Hypothesis Testing. Annual Review of Analytical Chemistry, Vol 9, 2016. 9: p. 449-472.

55. Chapman, J.D., D.R. Goodlett, and C.D. Masselon, Multiplexed and DataIndependent Tandem Mass Spectrometry for Global Proteome Profiling. Mass Spectrometry Reviews, 2014. 33(6): p. 452-470.

56. Mi, H.Y., et al., PANTHER version 10: expanded protein families and functions, and analysis tools. Nucleic Acids Research, 2016. 44(D1): p. D336-D342.

57. Bindea, G., et al., ClueGO: a Cytoscape plug-in to decipher functionally grouped gene ontology and pathway annotation networks. Bioinformatics, 2009. 25(8): p. 1091-1093.

58. Kramer, A., et al., Causal analysis approaches in Ingenuity Pathway Analysis. Bioinformatics, 2014. 30(4): p. 523-530.

59. Szklarczyk, D., et al., STRING v10: protein-protein interaction networks, integrated over the tree of life. Nucleic Acids Research, 2015. 43(D1): p. D447D452.

60. Gandhi, M.S., et al., Causes and consequences of zinc dyshomeostasis in rats with chronic aldosteronism. J Cardiovasc Pharmacol, 2008. 52(3): p. 245-52.

61. Shannon, P., et al., Cytoscape: a software environment for integrated models of biomolecular interaction networks. Genome Res, 2003. 13(11): p. 2498-504.

62. Mi, H., et al., Large-scale gene function analysis with the PANTHER classification system. Nat Protoc, 2013. 8(8): p. 1551-66.

63. Emanuelsson, O., et al., Predicting subcellular localization of proteins based on their N-terminal amino acid sequence. Journal of Molecular Biology, 2000.

300(4): p. 1005-1016.

64. Neupert, W. and J.M. Herrmann, Translocation of proteins into mitochondria. Annual Review of Biochemistry, 2007. 76: p. 723-749.

65. Ozcan, U., Mitofusins: Mighty Regulators of Metabolism. Cell, 2013. 155(1): p. 17-18.

66. Huang, J.Y., et al., Mitochondrial sirtuins. Biochim Biophys Acta, 2010. 1804(8): p. 1645-51.

67. Raman, D., et al., Characterization of Chemokine Receptor Cxcr2 Interacting Proteins Using a Proteomics Approach to Define the Cxcr2 "Chemosynapse". Chemokines, Pt A, 2009. 460: p. 315-+.

68. Thakur, D., et al., Microproteomic analysis of 10,000 laser captured microdissected breast tumor cells using short-range sodium dodecyl sulfatepolyacrylamide gel electrophoresis and porous layer open tubular liquid chromatography tandem mass spectrometry. Journal of Chromatography A, 2011. 1218(45): p. 8168-8174.

69. Paulo, J.A., et al., Short Gel, Long Gradient Liquid Chromatography Tandem Mass Spectrometry to Discover Urinary Biomarkers of Chronic Pancreatitis. Open Proteomics J, 2013. 6: p. 1-13. 
70. Sajic, T., Y.S. Liu, and R. Aebersold, Using data-independent, high-resolution mass spectrometry in protein biomarker research: Perspectives and clinical applications. Proteomics Clinical Applications, 2015. 9(3-4): p. 307-321.

71. Gillet, L.C., et al., Targeted Data Extraction of the MS/MS Spectra Generated by Data-independent Acquisition: A New Concept for Consistent and Accurate Proteome Analysis. Molecular \& Cellular Proteomics, 2012. 11(6).

72. Francois, G., et al., Image-based modeling of the human eye. IEEE Trans Vis Comput Graph, 2009. 15(5): p. 815-27.

73. Coroneo, M.T., N.W. Mullerstolzenburg, and A. Ho, Peripheral Light Focusing by the Anterior Eye and the Ophthalmohelioses. Ophthalmic Surgery and Lasers, 1991. 22(12): p. 705-711.

74. Cheng, H.Y., et al., Structural and functional MRI reveals multiple retinal layers. Proceedings of the National Academy of Sciences of the United States of America, 2006. 103(46): p. 17525-17530.

75. Lucas, R.J., et al., Regulation of the mammalian pineal by non-rod, non-cone, ocular photoreceptors. Science, 1999. 284(5413): p. 505-507.

76. LaVail, M.M., Outer segment disc shedding and phagocytosis in the outer retina. Trans Ophthalmol Soc U K, 1983. 103 ( Pt 4): p. 397-404.

77. Plafker, S.M., G.B. O'Mealey, and L.I. Szweda, Mechanisms for countering oxidative stress and damage in retinal pigment epithelium. Int Rev Cell Mol Biol, 2012. 298: p. 135-77.

78. Cook, H.L., P.J. Patel, and A. Tufail, Age-related macular degeneration: diagnosis and management. British Medical Bulletin, 2008. 85: p. 127-149.

79. Fritsche, L.G., et al., Age-Related Macular Degeneration: Genetics and Biology Coming Together. Annual Review of Genomics and Human Genetics, Vol 15, 2014. 15: p. 151-171.

80. Bressler, N.M., Early detection and treatment of neovascular age-related macular degeneration. J Am Board Fam Pract, 2002. 15(2): p. 142-52.

81. Klein, R., et al., Associations of Candidate Genes to Age-Related Macular Degeneration Among Racial/Ethnic Groups in the Multi-Ethnic Study of Atherosclerosis. American Journal of Ophthalmology, 2013. 156(5): p. 10101020.

82. Seddon, J.M., et al., Three New Genetic Loci (R1210C in CFH, Variants in COL8A1 and RAD51B) Are Independently Related to Progression to Advanced Macular Degeneration. Plos One, 2014. 9(1).

83. Khan, J.C., et al., Smoking and age related macular degeneration: the number of pack years of cigarette smoking is a major determinant of risk for both geographic atrophy and choroidal neovascularisation. British Journal of Ophthalmology, 2006. 90(1): p. 75-80.

84. Christen, W.G., et al., Dietary omega-3 fatty acid and fish intake and incident age-related macular degeneration in women. Arch Ophthalmol, 2011. 129(7): $\mathrm{p}$. 921-9.

85. Chen, Y.H., M. Bedell, and K. Zhang, Age-related Macular Degeneration: Genetic and Environmental Factors of Disease. Molecular Interventions, 2010. 10(5): p. 271-281. 
86. Mullins, R.F., N. Aptsiauri, and G.S. Hageman, Structure and composition of drusen associated with glomerulonephritis: implications for the role of complement activation in drusen biogenesis. Eye, 2001. 15: p. 390-395.

87. Klein, R.J., et al., Complement factor H polymorphism in age-related macular degeneration. Science, 2005. 308(5720): p. 385-389.

88. Edwards, A.O., et al., Complement factor H polymorphism and age-related macular degeneration. Science, 2005. 308(5720): p. 421-424.

89. Kassoff, A., et al., A randomized, placebo-controlled, clinical trial of high-dose supplementation with vitamins $C$ and $E$, beta carotene, and zinc for age-related macular degeneration and vision loss - AREDS Report No. 8. Archives of Ophthalmology, 2001. 119(10): p. 1417-1436.

90. Nandrot, E.F., et al., Loss of synchronized retinal phagocytosis and age-related blindness in mice lacking alphavbeta5 integrin. J Exp Med, 2004. 200(12): p. 1539-45.

91. Handa, J.T., et al., Lipids, oxidized lipids, oxidation-specific epitopes, and Agerelated Macular Degeneration. Biochimica Et Biophysica Acta-Molecular and Cell Biology of Lipids, 2017. 1862(4): p. 430-440.

92. Hageman, G.S. and R.F. Mullins, Molecular composition of drusen as related to substructural phenotype. Molecular Vision, 1999. 5(24-35).

93. Yamada, Y., et al., Oxidized low density lipoproteins induce a pathologic response by retinal pigmented epithelial cells. Journal of Neurochemistry, 2008. 105(4): p. 1187-1197.

94. Levitan, I., S. Volkov, and P.V. Subbaiah, Oxidized LDL: diversity, patterns of recognition, and pathophysiology. Antioxidants \& redox signaling, 2010. 13(1): p. 39-75.

95. Binder, C.J., N. Papac-Milicevic, and J.L. Witztum, Innate sensing of oxidationspecific epitopes in health and disease. Nature Reviews Immunology, 2016. 16(8): p. 485-497.

96. Curcio, C.A., et al., Aging, age-related macular degeneration, and the responseto-retention of apolipoprotein B-containing lipoproteins. Progress in Retinal and Eye Research, 2009. 28(6): p. 393-422.

97. Ebrahimi, K.B. and J.T. Handa, Lipids, lipoproteins, and age-related macular degeneration. J Lipids, 2011. 2011: p. 802059.

98. Komai, K. and A. Yoshimura, Role of scavenger receptors as damage-associated molecular pattern receptors in Toll-like receptor activation. Cytokine, 2017. 100: p. 125-126.

99. Ramet, M., et al., Drosophila scavenger receptor $\mathrm{Cl}$ is a pattern recognition receptor for bacteria. Immunity, 2001. 15(6): p. 1027-1038.

100. Davlyatshina, N., et al., Gene expression analysis of scavenger receptors class $B$ in pathogenesis of atherosclerosis. European Journal of Clinical Investigation, 2017. 47: p. 41-41.

101. PrabhuDas, M.R., et al., A Consensus Definitive Classification of Scavenger Receptors and Their Roles in Health and Disease. Journal of Immunology, 2017. 198(10): p. 3775-3789. 
102. Febbraio, M., D.P. Hajjar, and R.L. Silverstein, CD36: a class B scavenger receptor involved in angiogenesis, atherosclerosis, inflammation, and lipid metabolism. Journal of Clinical Investigation, 2001. 108(6): p. 785-791.

103. Pepino, M.Y., et al., Structure-Function of CD36 and Importance of Fatty Acid Signal Transduction in Fat Metabolism. Annual Review of Nutrition, Vol 34, 2014. 34: p. 281-303.

104. Asch, A.S., et al., Isolation of the thrombospondin membrane receptor. J Clin Invest, 1987. 79(4): p. 1054-61.

105. Ockenhouse, C.F., et al., Identification of a Platelet Membrane Glycoprotein as a Falciparum-Malaria Sequestration Receptor. Science, 1989. 243(4897): p. 14691471.

106. Nakata, A., et al., CD36, a novel receptor for oxidized low-density lipoproteins, is highly expressed on lipid-laden macrophages in human atherosclerotic aorta. Arteriosclerosis Thrombosis and Vascular Biology, 1999. 19(5): p. 1333-1339.

107. Navazo, M.D.P., et al., Identification on human CD36 of a domain (155-183) implicated in binding oxidized low-density lipoproteins (Ox-LDL). Arteriosclerosis Thrombosis and Vascular Biology, 1996. 16(8): p. 1033-1039.

108. Kar, N.S., et al., Mapping and characterization of the binding site for specific oxidized phospholipids and oxidized low density lipoprotein of scavenger receptor CD36. Journal of Biological Chemistry, 2008. 283(13): p. 8765-8771.

109. Coort, S.L.M., et al., Sulfo-N-succinimidyl esters of long chain fatty acids specifically inhibit fatty acid translocase (FAT/CD36)-mediated cellular fatty acid uptake. Molecular and Cellular Biochemistry, 2002. 239(1-2): p. 213-219.

110. Su, X. and N.A. Abumrad, Cellular fatty acid uptake: a pathway under construction. Trends in Endocrinology and Metabolism, 2009. 20(2): p. 72-77.

111. Park, Y.M., CD36, a scavenger receptor implicated in atherosclerosis. Experimental and Molecular Medicine, 2014. 46.

112. Ryeom, S.W., J.R. Sparrow, and R.L. Silverstein, CD36 participates in the phagocytosis of rod outer segments by retinal pigment epithelium. J Cell Sci, 1996. 109 ( Pt 2): p. 387-95.

113. Sun, M.J., et al., Light-induced oxidation of photoreceptor outer segment phospholipids generates ligands for CD36-mediated phagocytosis by retinal pigment epithelium - A potential mechanism for modulating outer segment phagocytosis under oxidant stress conditons. Journal of Biological Chemistry, 2006. 281(7): p. 4222-4230.

114. Picard, E., et al., CD36 plays an important role in the clearance of oxLDL and associated age-dependent sub-retinal deposits. Aging-Us, 2010. 2(12): p. 981989.

115. Gordiyenko, N., et al., RPE cells internalize low-density lipoprotein (LDL) and oxidized $L D L$ (oxLDL) in large quantities in vitro and in vivo. Investigative Ophthalmology \& Visual Science, 2004. 45(8): p. 2822-2829.

116. Arai, S., et al., A role for the apoptosis inhibitory factor AIM/Sp alpha/Api6 in atherosclerosis development. Cell Metabolism, 2005. 1(3): p. 201-213.

117. Yamazaki, T., et al., Circulating AIM as an Indicator of Liver Damage and Hepatocellular Carcinoma in Humans. Plos One, 2014. 9(10). 
118. Valledor, A.F., et al., Activation of liver $X$ receptors and retinoid $X$ receptors prevents bacterial-induced macrophage apoptosis. Proceedings of the National Academy of Sciences of the United States of America, 2004. 101(51): p. 1781317818.

119. Arai, S., et al., Obesity-associated autoantibody production requires AIM to retain the immunoglobulin $M$ immune complex on follicular dendritic cells. Cell Rep, 2013. 3(4): p. 1187-98.

120. Amezaga, N., et al., Human scavenger protein AIM increases foam cell formation and CD36-mediated oxLDL uptake. Journal of Leukocyte Biology, 2014. 95(3): p. 509-520.

121. Iannaccone, A., et al., Retinal pigment epithelium and microglia express the CD5 antigen-like protein, a novel autoantigen in age-related macular degeneration. Experimental Eye Research, 2017. 155: p. 64-74.

122. Chaum, E. and H. Yang, Transgenic expression of IGF-1 modifies the proliferative potential of human retinal pigment epithelial cells. Invest Ophthalmol Vis Sci, 2002. 43(12): p. 3758-64.

123. Bhattacharya, S., et al., Age-related susceptibility to apoptosis in human retinal pigment epithelial cells is triggered by disruption of p53-Mdm2 association. Invest Ophthalmol Vis Sci, 2012. 53(13): p. 8350-66.

124. Yamada, Y., et al., Oxidized low density lipoproteins induce a pathologic response by retinal pigmented epithelial cells. J Neurochem, 2008. 105(4): p. 1187-97.

125. Gnanaguru, G., et al., Oxidized Lipoprotein Uptake Through the CD36 Receptor Activates the NLRP3 Inflammasome in Human Retinal Pigment Epithelial Cells. Investigative Ophthalmology \& Visual Science, 2016. 57(11): p. 4704-4712.

126. Suzuki, M., et al., Oxidized phospholipids in the macula increase with age and in eyes with age-related macular degeneration. Molecular Vision, 2007. 13(80-85): p. 772-778.

127. Fliesler, S.J. and L. Bretillon, Thematic Review Series: Lipids and Lipid Metabolism in the Eye The ins and outs of cholesterol in the vertebrate retina. Journal of Lipid Research, 2010. 51(12): p. 3399-3413.

128. Kuda, O., et al., Sulfo-N-succinimidyl Oleate (SSO) Inhibits Fatty Acid Uptake and Signaling for Intracellular Calcium via Binding CD36 Lysine 164. Journal of Biological Chemistry, 2013. 288(22): p. 15547-15555.

129. Arai, S., et al., A role for the apoptosis inhibitory factor AIM/Spalpha/Api6 in atherosclerosis development. Cell Metab, 2005. 1(3): p. 201-13.

130. Sanjurjo, L., et al., CD5L Promotes M2 Macrophage Polarization through Autophagy-Mediated Upregulation of ID3. Frontiers in Immunology, 2018. 9.

131. Joseph, S.B., et al., LXR-dependent gene expression is important for macrophage survival and the innate immune response. Cell, 2004. 119(2): p. 299-309.

132. Valledor, A.F., et al., Activation of liver X receptors and retinoid X receptors prevents bacterial-induced macrophage apoptosis. Proc Natl Acad Sci U S A, 2004. 101(51): p. 17813-8.

133. Arai, S., et al., Apoptosis inhibitor of macrophage protein enhances intraluminal debris clearance and ameliorates acute kidney injury in mice. Nature Medicine, 2016. 22(2): p. 183-193. 
134. Friedman, D.S., et al., Prevalence of age-related macular degeneration in the United States. Arch Ophthalmol, 2004. 122(4): p. 564-72.

135. Nowak, J.Z., Age-related macular degeneration (AMD): pathogenesis and therapy. Pharmacol Rep, 2006. 58(3): p. 353-63.

136. Al-Zamil, W.M. and S.A. Yassin, Recent developments in age-related macular degeneration: a review. Clin Interv Aging, 2017. 12: p. 1313-1330.

137. Jang, K.H., et al., AIF-independent parthanatos in the pathogenesis of dry agerelated macular degeneration. Cell Death Dis, 2017. 8(1): p. e2526.

138. DeAngelis, M.M., et al., Genetics of age-related macular degeneration (AMD). Hum Mol Genet, 2017. 26(R2): p. R246.

139. Beatty, S., et al., The role of oxidative stress in the pathogenesis of age-related macular degeneration. Surv Ophthalmol, 2000. 45(2): p. 115-34.

140. Ratnayaka, J.A., L.C. Serpell, and A.J. Lotery, Dementia of the eye: the role of amyloid beta in retinal degeneration. Eye (Lond), 2015. 29(8): p. 1013-26.

141. Weigel, A.L., J.T. Handa, and L.M. Hjelmeland, Microarray analysis of $\mathrm{H}_{2} \mathrm{O} 2$-, $H N E-$, or tBH-treated ARPE-19 cells. Free Radical Biology and Medicine, 2002. 33(10): p. 1419-1432.

142. Cano, M., et al., Oxidative stress induces mitochondrial dysfunction and a protective unfolded protein response in RPE cells. Free Radical Biology and Medicine, 2014. 69: p. 1-14.

143. Koller, D., et al., Effects of Oxidized Phospholipids on Gene Expression in RAW 264.7 Macrophages: A Microarray Study. Plos One, 2014. 9(10).

144. Sherman, B.T. and R.A. Lempicki, Systematic and integrative analysis of large gene lists using DAVID bioinformatics resources. Nature protocols, 2009. 4(1): p. 44-57.

145. Gnanaguru, G., et al., Oxidized Lipoprotein Uptake Through the CD36 Receptor Activates the NLRP3 Inflammasome in Human Retinal Pigment Epithelial Cells. Invest Ophthalmol Vis Sci, 2016. 57(11): p. 4704-12.

146. Gordiyenko, N., et al., RPE cells internalize low-density lipoprotein (LDL) and oxidized $L D L$ (oxLDL) in large quantities in vitro and in vivo. Invest Ophthalmol Vis Sci, 2004. 45(8): p. 2822-9.

147. Kuda, O., et al., Sulfo-N-succinimidyl Oleate (SSO) Inhibits Fatty Acid Uptake and Signaling for Intracellular Calcium via Binding CD36 Lysine 164 SSO ALSO INHIBITS OXIDIZED LOW DENSITY LIPOPROTEIN UPTAKE BY MACROPHAGES. Journal of Biological Chemistry, 2013. 288(22): p. 1554715555.

148. Weismann, D., et al., Complement factor H binds malondialdehyde epitopes and protects from oxidative stress. Nature, 2011. 478(7367): p. 76-81.

149. Krönke, G., et al., Oxidized phospholipids induce expression of human heme oxygenase-1 involving activation of cAMP-responsive element-binding protein. Journal of Biological Chemistry, 2003. 278(51): p. 51006-51014.

150. Maines, M.D., The heme oxygenase system: a regulator of second messenger gases. Annual review of pharmacology and toxicology, 1997. 37(1): p. 517-554.

151. Wang, L.-J., et al., Expression of heme oxygenase-1 in atherosclerotic lesions. The American journal of pathology, 1998. 152(3): p. 711. 
152. Willis, D., A.R. Moore, and D.A. Willoughby, Heme oxygenase isoform expression in cellular and antibody-mediated models of acute inflammation in the rat. The Journal of pathology, 2000. 190(5): p. 627-634.

153. Bauer, M. and I. Bauer, Heme oxygenase-1: redox regulation and role in the hepatic response to oxidative stress. Antioxidants and Redox Signaling, 2002. 4(5): p. 749-758.

154. Lee, T.-S. and L.-Y. Chau, Heme oxygenase-1 mediates the anti-inflammatory effect of interleukin-10 in mice. Nature medicine, 2002. 8(3): p. 240.

155. Synowiec, E., et al., An association between polymorphism of the heme oxygenase-1 and-2 genes and age-related macular degeneration. Molecular Biology Reports, 2012. 39(3): p. 2081-2087.

156. Ambati, J., J.P. Atkinson, and B.D. Gelfand, Immunology of age-related macular degeneration. Nature Reviews Immunology, 2013. 13(6): p. 438-451.

157. Ferguson, T.A. and R.S. Apte, Angiogenesis in eye disease: immunity gained or immunity lost? Seminars in Immunopathology, 2008. 30(2): p. 111-119.

158. $\quad$ van Tits, L.J., et al., Oxidized LDL enhances pro-inflammatory responses of alternatively activated M2 macrophages: a crucial role for Kruppel-like factor 2. Atherosclerosis, 2011. 214(2): p. 345-9.

159. Kiyan, Y., et al., oxLDL induces inflammatory responses in vascular smooth muscle cells via urokinase receptor association with CD36 and TLR4. J Mol Cell Cardiol, 2014. 66: p. 72-82.

160. Chen, C. and D.B. Khismatullin, Oxidized low-density lipoprotein contributes to atherogenesis via co-activation of macrophages and mast cells. PLoS One, 2015. 10(3): p. e0123088.

161. Huang, J.D., et al., 7-Ketocholesterol-Induced Inflammation Signals Mostly through the TLR4 Receptor Both In Vitro and In Vivo. Plos One, 2014. 9(7).

162. Huang, J.D., et al., Sterculic acid antagonizes 7-ketocholesterol-mediated inflammation and inhibits choroidal neovascularization. Biochimica Et Biophysica Acta-Molecular and Cell Biology of Lipids, 2012. 1821(4): p. 637646.

163. Indaram, M., et al., 7-Ketocholesterol Increases Retinal Microglial Migration, Activation, and Angiogenicity: A Potential Pathogenic Mechanism Underlying Age-related Macular Degeneration. Scientific Reports, 2015. 5.

164. Hu, H.-M., et al., The C/EBP bZIP domain can mediate lipopolysaccharide induction of the proinflammatory cytokines interleukin-6 and monocyte chemoattractant protein-1. Journal of Biological Chemistry, 2000. 275(21): p. 16373-16381.

165. Singh, M. and S.C. Tyagi, Homocysteine mediates transcriptional changes of the inflammatory pathway signature genes in human retinal pigment epithelial cells. International Journal of Ophthalmology, 2017. 10(5): p. 696-704.

166. Kutty, R.K., et al., MicroRNA expression in human retinal pigment epithelial (ARPE-19) cells: Increased expression of microRNA-9 by N-(4-Hydroxyphenyl) retinamide. 2010.

167. Ruggiero, L. and S. Finnemann, Photoreceptor-RPE interactions: diurnal phagocytosis. The new visual neurosciences. MIT Press, Cambridge, MA Google Scholar, 2014. 
168. LaVail, M.M., Rod outer segment disk shedding in rat retina: relationship to cyclic lighting. Science, 1976. 194(4269): p. 1071-4.

169. Young, R.W. and D. Bok, Participation of the retinal pigment epithelium in the rod outer segment renewal process. J Cell Biol, 1969. 42(2): p. 392-403.

170. Mazzoni, F., H. Safa, and S.C. Finnemann, Understanding photoreceptor outer segment phagocytosis: use and utility of RPE cells in culture. Experimental eye research, 2014. 126: p. 51-60.

171. Guido, M.E., et al., Inner retinal circadian clocks and non-visual photoreceptors: novel players in the circadian system. Progress in neurobiology, 2010. 92(4): p. 484-504.

172. Karan, G., et al., Lipofuscin accumulation, abnormal electrophysiology, and photoreceptor degeneration in mutant ELOVL4 transgenic mice: a model for macular degeneration. Proceedings of the National Academy of Sciences of the United States of America, 2005. 102(11): p. 4164-4169.

173. Dorey, C.K., et al., Cell loss in the aging retina. Relationship to lipofuscin accumulation and macular degeneration. Invest Ophthalmol Vis Sci, 1989. 30(8): p. 1691-9.

174. Villegas-Perez, M.P., [Light exposure, lipofuscin and age-related macular degeneration]. Arch Soc Esp Oftalmol, 2005. 80(10): p. 565-8.

175. Fanjul-Moles, M.L. and G.O. Lopez-Riquelme, Relationship between Oxidative Stress, Circadian Rhythms, and AMD. Oxidative Medicine and Cellular Longevity, 2016.

176. Trammell, R.A., S. Verhulst, and L.A. Toth, Effects of Sleep Fragmentation on Sleep and Markers of Inflammation in Mice. Comparative Medicine, 2014. 64(1): p. 13-24.

177. Leproult, R., U. Holmback, and E. Van Canter, Circadian Misalignment Augments Markers of Insulin Resistance and Inflammation, Independently of Sleep Loss. Diabetes, 2014. 63(6): p. 1860-1869.

178. Maury, E., H.K. Hong, and J. Bass, Circadian disruption in the pathogenesis of metabolic syndrome. Diabetes \& Metabolism, 2014. 40(5): p. 338-346.

179. Wang, Q., et al., Regulation of Retinal Inflammation by Rhythmic Expression of MiR-146a in Diabetic Retina. Investigative Ophthalmology \& Visual Science, 2014. 55(6): p. 3986-3994.

180. Smit-McBride, Z., et al., Comparison of In Vivo Gene Expression Profiling of RPE/Choroid following Intravitreal Injection of Dexamethasone and Triamcinolone Acetonide. Journal of Ophthalmology, 2016.

181. Ko, C.H. and J.S. Takahashi, Molecular components of the mammalian circadian clock. Human Molecular Genetics, 2006. 15: p. R271-R277.

182. Lowrey, P.L. and J.S. Takahashi, Genetics of Circadian Rhythms in Mammalian Model Organisms. Genetics of Circadian Rhythms, 2011. 74: p. 175-230.

183. Jaeger, C. and S.A. Tischkau, Role of aryl hydrocarbon receptor in circadian clock disruption and metabolic dysfunction. Environmental health insights, 2016. 10: p. EHI. S38343.

184. Dikic, I., Proteasomal and autophagic degradation systems. Annual review of biochemistry, 2017. 86: p. 193-224. 
185. Jain, A., et al., p62/SQSTM1 is a target gene for transcription factor NRF2 and creates a positive feedback loop by inducing antioxidant response element-driven gene transcription. Journal of Biological Chemistry, 2010. 285(29): p. 2257622591.

186. Viiri, J., et al., p62/sequestosome 1 as a regulator of proteasome inhibitorinduced autophagy in human retinal pigment epithelial cells. Mol Vis, 2010. 16: p. 1399-414.

187. Park, S., et al., p62/SQSTM1 enhances NOD2-mediated signaling and cytokine production through stabilizing NOD2 oligomerization. PLoS One, 2013. 8(2): p. e57138.

188. Neale, B.M., et al., Genome-wide association study of advanced age-related macular degeneration identifies a role of the hepatic lipase gene (LIPC). Proc Natl Acad Sci U S A, 2010. 107(16): p. 7395-400.

189. Reynolds, R., B. Rosner, and J.M. Seddon, Serum lipid biomarkers and hepatic lipase gene associations with age-related macular degeneration. Ophthalmology, 2010. 117(10): p. 1989-95.

190. Fritsche, L.G., et al., Seven new loci associated with age-related macular degeneration. Nat Genet, 2013. 45(4): p. 433-9, 439e1-2.

191. Klein, R., et al., Lipids, lipid genes, and incident age-related macular degeneration: the three continent age-related macular degeneration consortium. Am J Ophthalmol, 2014. 158(3): p. 513-24 e3.

192. Aldred, E.M., C. Buck, and K. Vall, Chapter 27 - Problems with lipid metabolism, in Pharmacology, E.M. Aldred, C. Buck, and K. Vall, Editors. 2009, Churchill Livingstone: Edinburgh. p. 203-208.

193. Gonzalez, M.W. and M.G. Kann, Chapter 4: Protein interactions and disease. PLoS Comput Biol, 2012. 8(12): p. e1002819.

194. Aebersold, R., et al., The Biology/Disease-driven Human Proteome Project (B/DHPP): Enabling Protein Research for the Life Sciences Community. Journal of Proteome Research, 2013. 12(1): p. 23-27.

195. Semba, R.D., et al., The Human Eye Proteome Project: Perspectives on an emerging proteome. Proteomics, 2013. 13(16): p. 2500-2511.

196. Yilmaz, S., et al., A Pipeline for Differential Proteomics in Unsequenced Species. Journal of Proteome Research, 2016. 15(6): p. 1963-1970.

197. Kalli, A., et al., Evaluation and Optimization of Mass Spectrometric Settings during Data-dependent Acquisition Mode: Focus on LTQ-Orbitrap Mass Analyzers. Journal of Proteome Research, 2013. 12(7): p. 3071-3086.

198. Doerr, A., DIA mass spectrometry. Nature Methods, 2015. 12(1): p. 35-35.

199. Domanski, D., et al., MRM-based multiplexed quantitation of 67 putative cardiovascular disease biomarkers in human plasma. Proteomics, 2012. 12(8): p. 1222-43.

200. Wienkoop, S. and W. Weckwerth, Relative and absolute quantitative shotgun proteomics: targeting low-abundance proteins in Arabidopsis thaliana. Journal of Experimental Botany, 2006. 57(7): p. 1529-1535.

201. Silva, J.C., et al., Absolute quantification of proteins by LCMSE - A virtue of parallel MS acquisition. Molecular \& Cellular Proteomics, 2006. 5(1): p. 144156. 
202. Old, W.M., et al., Comparison of label-free methods for quantifying human proteins by shotgun proteomics. Molecular \& Cellular Proteomics, 2005. 4(10): p. 1487-1502.

203. Wang, X.N., et al., Feature extraction in the analysis of proteomic mass spectra. Proteomics, 2006. 6(7): p. 2095-2100.

204. Zhang, C. and Y. Liu, Retrieving Quantitative Information of Histone PTMs by Mass Spectrometry. Proteomics in Biology, Pt B, 2017. 586: p. 165-191.

205. Chernushevich, I.V., A.V. Loboda, and B.A. Thomson, An introduction to quadrupole-time-of-flight mass spectrometry. Journal of Mass Spectrometry, 2001. 36(8): p. 849-865.

206. Ebrahimi, K.B., et al., Decreased membrane complement regulators in the retinal pigmented epithelium contributes to age-related macular degeneration. Journal of Pathology, 2013. 229(5): p. 729-742.

207. Kamei, M., et al., Scavenger receptors for oxidized lipoprotein in age-related macular degeneration. Investigative Ophthalmology \& Visual Science, 2007. 48(4): p. 1801-1807.

208. Glenn, J.V., et al., Proteomic profiling of human retinal pigment epithelium exposed to an advanced glycation-modified substrate. Graefes Archive for Clinical and Experimental Ophthalmology, 2012. 250(3): p. 349-359.

209. Merl-Pham, J., F. Gruhn, and S.M. Hauck, Proteomic Profiling of Cigarette Smoke Induced Changes in Retinal Pigment Epithelium Cells. Retinal Degenerative Diseases: Mechanisms and Experimental Therapy, 2016. 854: p. 785-791.

210. Tababat-Khani, P., et al., Photocoagulation of human retinal pigment epithelium in vitro: unravelling the effects on ARPE-19 by transcriptomics and proteomics. Acta Ophthalmologica, 2015. 93(4): p. 348-354.

211. Kulak, N.A., et al., Minimal, encapsulated proteomic-sample processing applied to copy-number estimation in eukaryotic cells. Nature Methods, 2014. 11(3): p. 319-U300.

212. Distler, U., et al., Drift time-specific collision energies enable deep-coverage data-independent acquisition proteomics. Nature Methods, 2014. 11(2): p. 167-+.

213. Huang, D.W., B.T. Sherman, and R.A. Lempicki, Systematic and integrative analysis of large gene lists using DAVID bioinformatics resources. Nature Protocols, 2009. 4(1): p. 44-57.

214. Samuel, W., et al., Appropriately differentiated ARPE-19 cells regain phenotype and gene expression profiles similar to those of native RPE cells. Molecular Vision, 2017. 23: p. 60-89.

215. Zacchigna, S., et al., Loss of the Cholesterol-Binding Protein Prominin-1/CD133 Causes Disk Dysmorphogenesis and Photoreceptor Degeneration. Journal of Neuroscience, 2009. 29(7): p. 2297-2308.

216. Yang, Z.L., et al., Mutant prominin 1 found in patients with macular degeneration disrupts photoreceptor disk morphogenesis in mice (vol 118, pg 2908, 2008). Journal of Clinical Investigation, 2009. 119(5): p. 1396-1396.

217. Voillet, V., et al., Integrated Analysis of Proteomic and Transcriptomic Data Highlights Late Fetal Muscle Maturation Process. Molecular \& Cellular Proteomics, 2018. 17(4): p. 672-693. 
218. Nie, L., et al., Integrated analysis of transcriptomic and proteomic data of Desulfovibrio vulgaris: zero-inflated Poisson regression models to predict abundance of undetected proteins. Bioinformatics, 2006. 22(13): p. 1641-1647.

219. Piruzian, E., et al., Integrated network analysis of transcriptomic and proteomic data in psoriasis. Bmc Systems Biology, 2010. 4.

220. Haider, S. and R. Pal, Integrated Analysis of Transcriptomic and Proteomic Data. Current Genomics, 2013. 14(2): p. 91-110.

221. Ghazalpour, A., et al., Comparative analysis of proteome and transcriptome variation in mouse. PLoS Genet, 2011. 7(6): p. e1001393.

222. Milo, R. and R. Phillips, Cell biology by the numbers. 2016.

223. Greenbaum, D., et al., Comparing protein abundance and $m R N A$ expression levels on a genomic scale. Genome Biol, 2003. 4(9): p. 117.

224. Tababat-Khani, P., et al., Photocoagulation of human retinal pigment epithelium in vitro: unravelling the effects on ARPE-19 by transcriptomics and proteomics. Acta Ophthalmol, 2015. 93(4): p. 348-54. 


\section{VITA}

Diwa Koirala was born in 1985 in Okhaldhunga, Nepal. She completed her Bachelors degree in Microbiology in 2006 from Tri Chandra College, Tribhuwan University, Kathmandu Nepal. She earned her Master's Degree in Pharmaceutical Sciences from Shanghai Jiao Tong University, Shanghai, China. Her master's thesis focused on development of new anticancer drug screening assay and development of a selective estrogen receptor modulator that suppresses tamoxifen-resistant tumor growth and targeting telomerase promoter. In the fall of 2012, she enrolled in the Pharmaceutical Sciences PhD program at the University of Tennessee Health Sciences Center, Memphis under the supervision of Dr. Sarka Beranova-Giorgianni. During her research at UTHSC, she focused on bioanalytical platforms for protein identification by mass spectrometry and interrogate of scavenger receptors role in retinal homeostasis. 